\title{
The "hit" problem of five variables in the generic degree and its application
}

\author{
Đặng Võ Phúc \\ Faculty of Education Studies, University of Khanh Hoa, \\ 01 Nguyen Chanh, Nha Trang, Khanh Hoa, Viet Nam \\ (Dedicated to Professor James F. Peters)
}

\begin{abstract}
Let $P_{s}:=\mathbb{F}_{2}\left[x_{1}, x_{2}, \ldots, x_{s}\right]$ be the graded polynomial algebra over the prime field of two elements, $\mathbb{F}_{2}$, in $s$ variables $x_{1}, x_{2}, \ldots, x_{s}$, each of degree one. This algebra is considered as a graded module over the mod-2 Steenrod algebra, $\mathscr{A}$. We are interested in the "hit" problem of finding a minimal set of generators for $\mathscr{A}$-module $P_{s}$. This problem is unresolved for every $s \geqslant 5$. In this paper, we study the hit problem of five variables in a generic degree, from which we investigate Singer's conjecture [Math. Z. 202 (1989), 493-523] for the transfer homomorphism of rank 5 in degrees given. This gives an efficient method to study the algebraic transfer and it is different from the ones of Singer.
\end{abstract}

Keywords:

Steenrod algebra; Peterson hit problem; Algebraic transfer; Steenrod squares; Invariant theory

2010 MSC: Primary 55S10; Secondary 55S05, 55T15.

\section{Contents}

1 Introduction and main results 1

2 Background 4

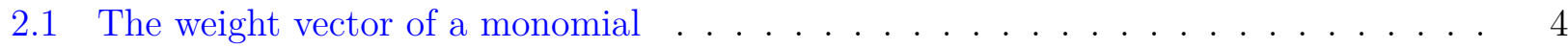

2.2 The admissible monomial . . . . . . . . . . . . . . . . . . . 4

$3 \mathscr{A}$-generators for $P_{5}$ in degree $3\left(2^{t}-1\right)+2^{t} \quad 7$

3.1 Singer's criterion on the hit monomials . . . . . . . . . . . . 7

3.2 Proof of Theorem $1.1 \ldots \ldots \ldots \ldots \ldots$

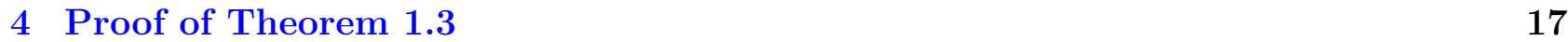

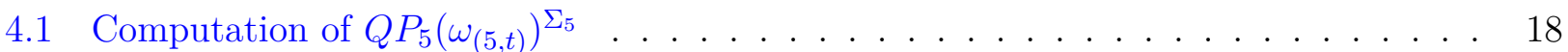

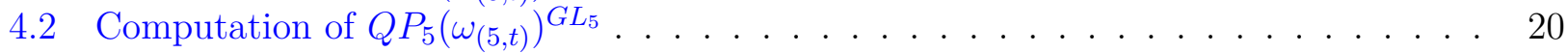

5 Appendix 21

5.1 The admissible monomials of degree $3\left(2^{t}-1\right)+2^{t}$ in $P_{5}^{0} \ldots \ldots \ldots \ldots 21$

5.2 The admissible monomials of degree $3\left(2^{t}-1\right)+2^{t}$ in $P_{5}^{+} \ldots \ldots \ldots . \ldots 24$

\section{Introduction and main results}

Let $\mathscr{A}$ denote the mod-2 Steenrod algebra. This algebra is defined to be the graded algebra over the prime field $\mathbb{F}_{2}$, generated by the Steenrod squares $S q^{i}$, in grading $i \geqslant 0$, subject to the Adem

Email address: dangphuc150488@gmail.com, dangvophuc@ukh.edu.vn (Đặng Võ Phúc) 
relations and $S q^{0}=1$ (see Steenrod and Epstein [43]). From a topological point of view, the Steenrod algebra is the algebra of stable cohomology operations for ordinary cohomology $H^{*}$ over $\mathbb{F}_{2}$. Let us denote by $P_{s}:=\mathbb{F}_{2}\left[x_{1}, x_{2}, \ldots, x_{s}\right]=\bigoplus_{d \geqslant 0}\left(P_{s}\right)_{d}$ the polynomial algebra over $\mathbb{F}_{2}$, which can be regarded as a graded left module over $\mathscr{A}$. The grading is by the degree of the homogeneous terms $\left(P_{s}\right)_{d}$ of degree $d$ in $s$ variables with the degree of each $x_{i}$ being 1 . The algebra $P_{s}$ arises as the cohomology with $\mathbb{F}_{2}$-coefficients of the elementary abelian 2-group $\mathbb{F}_{2}^{s}$ of rank $s$. As well known, the Steenrod algebra acts by the composition of linear operators on $P_{s}$ and the action of the Steenrod squares $S q^{i}:\left(P_{s}\right)_{d} \rightarrow\left(P_{s}\right)_{d+i}$ is determined by the Cartan formula and its elementary properties (see also [43], [56]).

A homogeneous element $f \in\left(P_{s}\right)_{d}$ in $\mathscr{A}$-module $P_{s}$ is hit if there is a finite sum $f=\sum_{j>0} S q^{j}\left(f_{j}\right)$, where the homogeneous elements $f_{j} \in P_{s}$ have grading strictly less than $d$. Let us denote by $Q P_{s}:=\mathbb{F}_{2} \otimes_{\mathscr{A}} P_{s}$ the quotient of the left $\mathscr{A}$-module $P_{s}$ by the hit elements in $\mathscr{A}^{+} \cdot P_{s}$, where $\mathscr{A}^{+}$ denotes the augmentation ideal of $\mathscr{A}$ and $\mathbb{F}_{2}$ is viewed as a right $\mathscr{A}$-module concentrated in grading 0 . Then, $Q P_{s}$ is a graded vector space over $\mathbb{F}_{2}$ and a basis for $Q P_{s}$ lifts to a minimal generating set for $P_{s}$ as a module over $\mathscr{A}$. It should be noted that this space is also considered as a form modular representation of $G L_{s}$ over $\mathbb{F}_{2}$. We are interested in the hit problem of finding a monomial basis of $Q P_{s}$ in each $s$ and degree $n \geqslant 0$. This is the same as the problem of determining of a minimal set of generators for $\mathscr{A}$-module $P_{s}$. Peterson [23], Wood [55], Singer [41], and Priddy [37] laid the first foundation for the study of the hit problem and pointed out its relationship to several classical problems in the homotopy theory such as cobordism theory of manifolds, modular representation theory of the general linear group, and stable homotopy type of classifying spaces of finite groups. Later, many researchers have been interested in discovering these hit problems (see the works of Boardman [2], Bruner-Hà-Hưng [4], Crabb and Hubbuck [5], Hưng [11], Kameko [12], Silverman [39], Silverman and Singer [40], Walker and Wood [52, 53, 54], the present author [24]-[32], Sum [44]-[50] and others), but it was a well known unresolved problem for $s \geqslant 5$.

For a non-negative integer $d$, denote by $\left(Q P_{s}\right)_{d}$ the subspace of $Q P_{s}$ consisting of all the classes represented by the homogeneous polynomials in $\left(P_{s}\right)_{d}$. One of the extremely useful tools for studying the hit problem is Kameko's homomorphism [12]: $\widetilde{S q_{*}^{0}}=\left(\widetilde{S q_{*}^{0}}\right)_{(s, 2 d+s)}:\left(Q P_{s}\right)_{2 d+s} \rightarrow\left(Q P_{s}\right)_{d}$, which is induced by the $\mathbb{F}_{2}$-linear map $\psi: P_{s} \longrightarrow P_{s}$, given by

$$
\psi\left(x_{1}^{n_{1}} x_{2}^{n_{2}} \ldots x_{s}^{n_{s}}\right)= \begin{cases}x_{1}^{\frac{n_{1}-1}{2}} x_{2}^{\frac{n_{2}-1}{2}} \ldots x_{s}^{\frac{n_{s}-1}{2}} & \text { if } n_{1}, n_{2}, \ldots, n_{s} \text { odd }, \\ 0 & \text { otherwise }\end{cases}
$$

for any monomial $x_{1}^{n_{1}} x_{2}^{n_{2}} \ldots x_{s}^{n_{s}} \in P_{s}$. It is straightforward to see that $\psi$ is not an $\mathscr{A}$-homomorphism, but $\psi S q^{2 i}=S q^{i} \psi$ and $\psi S q^{2 i+1}=0$ for any $i \geqslant 0$. The structure of $Q P_{s}$ was systematically studied for the cases $s \leqslant 4$ (see [2,12, 23, 47]). Specifically, by the works of Wood [55] and Sum [50], it is sufficient to determine $Q P_{s}$ at each degree $d$ of the form:

$$
d=r\left(2^{t}-1\right)+2^{t} m
$$

whenever $r, t, m$ are non-negative integers satisfying $1 \leqslant r=\mu(d) \leqslant s$ and $\mu(m)<r$ (see Subsection 2.2). Here, $\mu(n)$ denotes the smallest number $k$ such that $n$ can be written in the form $\sum_{1 \leqslant i \leqslant k}\left(2^{u_{i}}-1\right)$, where $u_{i}>0$. For $r=s-1$, the problem was investigated by Crabb and Hubbuck [5], Nam [21], Repka and Selick [38], Mothebe [19], Sum [45, 47] and by us [24, 25]. For $r=s=5$, it is known by Sum [46, 50] with $m=5,10$ and by the present writer [26]-[32] with $m=6,8,18,22,42$. So far there is no a completely answer for the case $s=5$. It should be noted that solving special cases plays an important role in finding general solutions to the problem. By these reasons, our motivation to write up the present paper is to continue studying the hit problem in the 5-variable case. More specifically, we will explicitly describe $Q P_{5}$ at degrees in (1.1) when $r=s-2=3$ and $m=1$. 
We begin our work with the following, which is one of our main results.

Theorem 1.1. Let $d=3\left(2^{t}-1\right)+2^{t}$ with $t$ a positive integer. The dimension of the $\mathbb{F}_{2}$-vector space $\left(Q P_{5}\right)_{d}$ is determined by the following table:

\begin{tabular}{c|cccccc}
$d=3\left(2^{t}-1\right)+2^{t}$ & $t=1$ & $t=2$ & $t=3$ & $t=4$ & $t=5$ & $t \geqslant 6$ \\
\hline $\operatorname{dim}\left(Q P_{5}\right)_{d}$ & 46 & 250 & 645 & 945 & 1115 & 1116
\end{tabular}

Noting that if $t=0$ then $d=3\left(2^{t}-1\right)+2^{t}=1$, and we can easily claim that $\left(Q P_{5}\right)_{1}$ has dimension 5 . The theorem has been proven by Sum [46, 50] for $t=1$ and by Moetele-Mothebe [20] for $t=2$. However, in this article, we prove the case $t=2$ in another way (see Subsection 3.2). Now, it should be noticed that Kameko's map $\widetilde{S q_{*}^{0}}=\left(\widetilde{S q_{*}^{0}}\right)_{(5, d)}:\left(Q P_{5}\right)_{d} \longrightarrow\left(Q P_{5}\right)_{\frac{d-5}{2}}$ is an epimorphism of $\mathbb{F}_{2}$-vector spaces. From this and our previous results $[24,25]$, in order to prove the theorem, we only need to determine the kernel of $\left(\widetilde{S q_{*}^{0}}\right)_{(5, d)}$. The calculations are quite long and complicated. Our method is based on the Kameko homomorphism and our recent results in [24, 25].

Let $G L_{s}:=G L\left(s, \mathbb{F}_{2}\right)$ be the general linear group of rank $s$ over $\mathbb{F}_{2}$. As it is known, this $G L_{s}$ acts regularly on $P_{s}$ by matrix substitution. Further, the two actions of $\mathscr{A}$ and $G L_{s}$ upon $P_{s}$ commute with each other; hence there is an inherited action of $G L_{s}$ on $Q P_{s}$. This shows that the problem we are interested in is one of the useful tools for studying the modular representation of $G L_{s}$ over $\mathbb{F}_{2}$. Moreover, it is applied to describe the $\mathbb{F}_{2}$-cohomology of the Steenrod algebra, Ext ${ }_{\mathscr{A}}^{* * *}\left(\mathbb{F}_{2}, \mathbb{F}_{2}\right)$ through Singer's transfer homomorphism [41]. Computing explicitly this cohomology is one of the problems of great importance in stable homotopy theory. But despite intensive investigation for nearly half a century, its structure remains elusive. The Singer transfer [41] is a linear transformation $\varphi_{s}: \operatorname{Tor}_{s, s+*}^{\mathscr{A}}\left(\mathbb{F}_{2}, \mathbb{F}_{2}\right) \longrightarrow\left(Q P_{s}\right)_{*}^{G L_{s}}$, from the $s$-th homology group of $\mathscr{A}$ to the space of $G L_{s^{-}}$ invariants of $\left(Q P_{s}\right)_{*}$. Dually, let $P H_{*}\left(\mathbb{F}_{2}^{s}\right)$ be the subspace of $H_{*}\left(\mathbb{F}_{2}^{s}\right)$ consisting of all elements that are annihilated by all positive degree Steenrod squares, then there is an induced action of $G L_{s}$ on $P H_{*}\left(\mathbb{F}_{2}^{s}\right)$, and we have an $\mathbb{F}_{2}$-linear map from the coinvariant elements of $P H_{*}\left(\mathbb{F}_{2}^{s}\right)$ to $\bmod -2$ cohomology group $\operatorname{Ext}_{\mathscr{A}}^{s, s+*}\left(\mathbb{F}_{2}, \mathbb{F}_{2}\right)$ of the Steenrod algebra, which is induced over the $E_{2}$-term of the Adams spectral sequence by the geometrical transfer map $\Sigma^{\infty}\left(B\left(\mathbb{F}_{2}^{s}\right)_{+}\right) \longrightarrow \Sigma^{\infty}\left(S^{0}\right)$ in stable homotopy theory (see also Mitchell [18]). These transfers can be played a key role in the study of the Kervaire invariant one problem (see Browder [3], Mahowald [15], Minami [16, 17]). The Kervaire invariant was first introduced by Browder's work [3], where he shows that the classes $h_{j}^{2} \in \operatorname{Ext}_{\mathscr{A}}^{2,2^{j+1}}\left(\mathbb{F}_{2}, \mathbb{F}_{2}\right)$ are the permanent cycles in the classical Adams spectral sequence at the prime 2, if and only if smooth framed manifolds of Kervaire invariant one exist only in dimensions $2^{j+1}-2$. The hypothetical element in stable $2^{j+1}-2$-stem, $\pi_{2^{j+1}-2}^{S}$, represented by such a framed manifold is denoted by $\theta_{j}$. These $\theta_{j}$ are known to be exist for $j \leqslant 5$ (see also Lin-Mahowald [13]). Many open issues in Algebraic and Differential Topology depend on knowing whether or not the Kervaire invariant one elements $\theta_{j}$ for $j \geqslant 6$. In 2016, Hill, Hopkins, and Ravenel [10] indicated that these elements do not exist for $j \geqslant 7$. So far the case $j=6$ is still no answer. Thus, smooth framed manifolds of Kervaire invariant one therefore exist only in dimensions 2,6,14,30,62, and possibly 126 .

Going back to the Singer transfer, in [41], Singer claimed that $\varphi_{s}$ is an isomorphism for $s=$ 1,2 and the "total" dual algebraic transfer $\bigoplus_{s \geqslant 0} \varphi_{s}^{*}$ is an algebra homomorphism. Afterwards, Boardman [2], with additional calculations by Kameko [12], showed that $\varphi_{3}$ is also an isomorphism. Additionally, in higher rank, Singer states that the transfer homomorphism is not a monomorphism in bidegree $(5,14)$ and made the following conjecture.

Conjecture 1.2. The homological transfer $\varphi_{s}$ is an epimorphism for any $s>0$.

This prediction is known to be true for $s \leqslant 3$. After more than 3 decades, we have recently proved in [36] that it is also true for $s=4$. The general case is still an open problem. Recently, some authors have been studied the conjecture for $s=4,5$ (see Bruner-Hà-Hưng [4], Hưng [11], Chơn-Hà [7, 8], Hà [9], Nam [22], the present author [26], [28]-[35], Sum [46, 48, 49, 50] and others). In the present work, by using techniques of the hit problem of five variables, we investigate Conjecture 1.2 in 
bidegree $(5, d+5)$, where $d$ is determined as in Theorem 1.1. This gives an efficient method to study the Singer transfer and it is different from that of Singer [41]. More explicitly, based on this approach, we obtain the following.

Theorem 1.3. For each positive integer $t$, the space of $G L_{5}$-invariants $\left(Q P_{5}\right)_{3\left(2^{t}-1\right)+2^{t}}^{G L_{5}}$ is trivial.

It is known that Kameko's map $\widetilde{S q_{*}^{0}}=\left(\widetilde{S q_{*}^{0}}\right)_{\left(5,3\left(2^{t}-1\right)+2^{t}\right)}:\left(Q P_{5}\right)_{3\left(2^{t}-1\right)+2^{t}} \longrightarrow\left(Q P_{5}\right)_{2^{t+1}-4}$ is an epimorphism of $G L_{5}$-modules. On the other side, following [48], $\left(Q P_{5}\right)_{2^{t+1}-4}^{G L_{5}}$ is trivial, for all $t>0$. So, to prove this theorem, we only need to determine the invariant $\left(\operatorname{Ker}\left(\left(\widetilde{S q_{*}^{0}}\right)_{\left(5,3\left(2^{t}-1\right)+2^{t}\right)}\right)\right)^{G L_{5}}$. The computations are based on a monomial basis of $\left(Q P_{5}\right)_{3\left(2^{t}-1\right)+2^{t}}$, which are given in the proof of Theorem 1.1.

By using calculations by Chen [6], Lin [14] and Tangora [51] and passing to the dual, deduce that $\operatorname{Tor}_{5,2^{t+2}+2}^{\mathscr{A}}\left(\mathbb{F}_{2}, \mathbb{F}_{2}\right)=0$ for all $t>0$. This together with Theorem 1.3 imply that the homological transfer $\varphi_{5}$ : $\operatorname{Tor}_{5,2^{t+2}+2}^{\mathscr{A}}\left(\mathbb{F}_{2}, \mathbb{F}_{2}\right) \rightarrow\left(Q P_{5}\right)_{3\left(2^{t}-1\right)+2^{t}}^{G L_{5}}$ is an isomorphism. As a consequence, we have immediately the following.

Corollary 1.4. Conjecture 1.2 is true for the rank 5 and the degree $3\left(2^{t}-1\right)+2^{t}$ with $t$ an arbitrary positive integer.

The paper contains five sections and is organized as follows: Background is provided in Section 2. The proofs of Theorem 1.1 and 1.3 will be presented in Sections 3 and 4, respectively. All the admissible monomials of degree $d$ in Theorem 1.1 are described in the Appendix.

\section{Acknowledgment}

This research is funded by Vietnam's National Foundation for Science and Technology Development (NAFOSTED) under grant number 101.04-2017.05.

The author appreciates anonymous referees for their careful corrections to, valuable comments on, and helpful suggestions to the last version of this paper.

The author would also like to express my profound gratitude to Professor Nguyen Sum for pointing out some errors in the original paper and his help in this work.

\section{Background}

In this section, we provide some known basis concepts, which might be useful in the next sections.

\subsection{The weight vector of a monomial}

Let us denote by $\alpha_{j}(d)$ the $j$-th coefficients in dyadic expansion of a non-negative integer $d$. Then, we have $d=\alpha_{0}(d) 2^{0}+\alpha_{1}(d) 2^{1}+\cdots+\alpha_{j}(d) 2^{j}+\cdots$, for $\alpha_{j}(d) \in\{0,1\}$ with $j \geqslant 0$. For a monomial $u=x_{1}^{a_{1}} x_{2}^{a_{2}} \ldots x_{s}^{a_{s}} \in P_{s}$, we define two sequences associated with $u$ by

$$
\begin{aligned}
\omega(u) & =\left(\omega_{1}(u), \omega_{2}(u), \ldots, \omega_{j}(u), \ldots\right), \\
\sigma(u) & =\left(a_{1}, a_{2}, \ldots, a_{s}\right),
\end{aligned}
$$

where $\omega_{j}(u):=\sum_{1 \leqslant i \leqslant s} \alpha_{j-1}\left(a_{i}\right) \leqslant s$, for all $j$. The sequences $\omega(u)$ and $\sigma(u)$ are called the weight vector and the exponent vector of the monomial $u$, respectively.

Let $\omega=\left(\omega_{1}, \omega_{2}, \ldots, \omega_{i}, \ldots\right)$ be a sequence of non-negative integers. The sequence $\omega$ is called a weight vector if $\omega_{j}=0$ for $j \gg 0$. By convention, the sets of all the weight vectors and the exponent vectors are given the left lexicographical order.

\subsection{The admissible monomial}

For a weight vector $\omega$, we define $\operatorname{deg} \omega=\sum_{i \geqslant 1} 2^{i-1} \omega_{i}$. Let us denote by $P_{s}(\omega)$ the subspace of $P_{s}$ spanned by all monomials $x \in P_{s}$ such that $\operatorname{deg}(x)=\operatorname{deg}(\omega), \omega(x) \leqslant \omega$, and by $P_{s}^{-}(\omega)$ the subspace of $P_{s}$ spanned by all monomials $x \in P_{s}$ such that $\operatorname{deg}(x)=\operatorname{deg}(\omega), \omega(x)<\omega$. 
Definition 2.2.1 (see [12], [47]). Let $\omega$ be a weight vector and let $f, g$ be two homogeneous polynomials of the same degree in $P_{s}$.

(i) $f \equiv g$, if and only if $(f+g) \in \mathscr{A}^{+} \cdot P_{s}$. When $f \equiv 0$, then $f \in \mathscr{A}^{+} \cdot P_{s}$.

(ii) $f \equiv_{\omega} g$ if and only if $f, g \in P_{s}(\omega)$ and $(f+g) \in\left(\left(\mathscr{A}^{+} \cdot P_{s} \cap P_{s}(\omega)\right)+P_{s}^{-}(\omega)\right)$. Specifically, $f$ is said to be $\omega$-hit, if $f \equiv_{\omega} 0$.

The Definition 2.2.1(ii) is a modification of a definition in Sum [47, Definition 2.3]. It can be easily seen that the binary relations $\equiv$ and $\equiv_{\omega}$ on $P_{s}$ are equivalence ones.

Definition 2.2.2 (see [12]). Let $x$ and $y$ be two monomials of the same degree in $P_{s}$. We say that $x<y$ if and only if one of the following holds:

(i) $\omega(x)<\omega(y)$;

(ii) $\omega(x)=\omega(y)$ and $\sigma(x)<\sigma(y)$.

Our key instrument here is the following notion.

Definition 2.2.3 (see [12], [47]). We say that a monomial $x$ in $P_{s}$ is inadmissible, if there exist monomials $y_{1}, y_{2}, \ldots, y_{m}$ such that $y_{t}<x$ for $1 \leqslant t \leqslant m$ and $x \equiv \sum_{1 \leqslant t \leqslant m} y_{t}$. Then, $x$ is said to be admissible, if it is not inadmissible.

Thus, the above definitions indicated that the set of all the admissible monomials of degree $d$ in $P_{s}$ is a minimal set of $\mathscr{A}$-generators for $P_{s}$ in degree $d$. Therefore, $\left(Q P_{s}\right)_{d}$ is an $\mathbb{F}_{2}$-vector space with a basis consisting of all the classes represented by the admissible monomials of degree $d$ in $P_{s}$.

Definition 2.2.4 (see [12], [47]). A monomial $x$ in $P_{s}$ is said to be strictly inadmissible if and only if there exists monomials $y_{1}, y_{2}, \ldots, y_{m}$ such that $y_{t}<x$ for $1 \leqslant t \leqslant m$ and

$$
x=\sum_{1 \leqslant t \leqslant m} y_{t}+\sum_{1 \leqslant j \leqslant 2^{r}-1} S q^{j}\left(h_{j}\right),
$$

where $r=\max \left\{i \in \mathbb{Z}: \omega_{i}(x)>0\right\}$ and suitable polynomials $h_{j} \in P_{s}$.

Noticing that if $x$ is strictly inadmissible, then it is inadmissible. However, in general, the converse is not true. For instance, the monomial $x=x_{1} x_{2}^{2} x_{3}^{2} x_{4}^{2} x_{5}^{2} x_{6}$ in $P_{6}$ is inadmissible, but it is not strictly inadmissible.

Theorem 2.2.5 (see [12], [47]). Let $x, y$ and $u$ be monomials in $P_{s}$ such that $\omega_{i}(x)=0$ for $i>r>0, \omega_{t}(u) \neq 0$ and $\omega_{i}(u)=0$ for $i>t>0$.

(i) If $u$ is inadmissible, then $x u^{2^{r}}$ is also inadmissible.

(ii) If $u$ is strictly inadmissible, then $u y^{2^{t}}$ is also strictly inadmissible.

From now on, we use the following notations.

$$
\begin{aligned}
P_{s}^{0} & =\left\langle\left\{x=x_{1}^{a_{1}} x_{2}^{a_{2}} \ldots x_{s}^{a_{s}} \in P_{s} \mid a_{1} a_{2} \ldots a_{s}=0\right\}\right\rangle, \\
P_{s}^{+} & =\left\langle\left\{x=x_{1}^{a_{1}} x_{2}^{a_{2}} \ldots x_{s}^{a_{s}} \in P_{s} \mid a_{1} a_{2} \ldots a_{s}>0\right\}\right\rangle .
\end{aligned}
$$

Then $P_{s}^{0}$ and $P_{s}^{+}$are the $\mathscr{A}$-submodules of $P_{s}$. Furthermore, we have a direct summand decomposition of the $\mathbb{F}_{2}$-vector spaces: $Q P_{s}=Q P_{s}^{0} \oplus Q P_{s}^{+}$, where $Q P_{s}^{0}:=\mathbb{F}_{2} \otimes_{\mathscr{A}} P_{s}^{0}$ and $Q P_{s}^{+}:=\mathbb{F}_{2} \otimes_{\mathscr{A}} P_{s}^{+}$.

Let $Q P_{s}(\omega)$ be the quotient of $P_{s}(\omega)$ by the equivalence relation $\equiv_{\omega}$. According to Walker and Wood [54], we have a filtration of $Q P_{s}$ :

$\{0\} \subseteq \cdots \subseteq P_{s}^{-}(\omega) /\left(\left(\mathscr{A}^{+} \cdot P_{s}\right) \cap P_{s}^{-}(\omega)\right) \subseteq P_{s}(\omega) /\left(\left(\mathscr{A}^{+} \cdot P_{s}\right) \cap P_{s}(\omega)\right) \subseteq \cdots \subseteq P_{s} /\left(\mathscr{A}^{+} \cdot P_{s}\right)=Q P_{s}$.

It should be noticed that this is not only a filtration of $Q P_{s}$ as a vector space, but also as a $G L_{s}$-module. The inclusion of $P_{s}^{-}(\omega)$ into $P_{s}(\omega)$ induces the monomorphism

$$
P_{s}^{-}(\omega) /\left(\left(\mathscr{A}^{+} \cdot P_{s}\right) \cap P_{s}^{-}(\omega)\right) \rightarrow P_{s}(\omega) /\left(\left(\mathscr{A}^{+} \cdot P_{s}\right) \cap P_{s}(\omega)\right)
$$


and the sequence is short exact:

$0 \rightarrow P_{s}^{-}(\omega) /\left(\left(\mathscr{A}^{+} \cdot P_{s}\right) \cap P_{s}^{-}(\omega)\right) \rightarrow P_{s}(\omega) /\left(\left(\mathscr{A}^{+} \cdot P_{s}\right) \cap P_{s}(\omega)\right) \rightarrow P_{s}(\omega) /\left(\left(\left(\mathscr{A}^{+} \cdot P_{s}\right) \cap P_{s}(\omega)\right)+P_{s}^{-}(\omega)\right) \rightarrow 0$.

Combining this and Definition 2.2.1(ii), one gets

$Q P_{s}(\omega)=P_{s}(\omega) /\left(\left(\mathscr{A}^{+} \cdot P_{s} \cap P_{s}(\omega)\right)+P_{s}^{-}(\omega)\right) \cong\left(P_{s}(\omega) /\left(\left(\mathscr{A}^{+} \cdot P_{s}\right) \cap P_{s}(\omega)\right)\right) /\left(P_{s}^{-}(\omega) /\left(\left(\mathscr{A}^{+} \cdot P_{s}\right) \cap P_{s}^{-}(\omega)\right)\right)$.

Following [50], $Q P_{s}(\omega)$ is also an $G L_{s}$-module, further this isomorphism is also an isomorphism of $G L_{s}$-modules. Combining this and the above filtration of $Q P_{s}$, we have immediately

$$
\operatorname{dim}\left(\left(Q P_{s}\right)_{d}\right)=\sum_{\operatorname{deg}(\omega)=d} \operatorname{dim}\left(Q P_{s}(\omega)\right), \operatorname{dim}\left(\left(Q P_{s}\right)_{d}^{G L_{s}}\right) \leqslant \sum_{\operatorname{deg}(\omega)=d} \operatorname{dim}\left(Q P_{s}(\omega)^{G L_{s}}\right) .
$$

Throughout this paper, we will always denote the set of all admissible monomials of degree $d$ in $P_{s}$ by $\mathscr{B}_{s}(d)$. We put

$$
\mathscr{B}_{s}^{0}(d):=\mathscr{B}_{s}(d) \cap\left(P_{s}^{0}\right)_{d}, \mathscr{B}_{s}^{+}(d):=\mathscr{B}_{s}(d) \cap\left(P_{s}^{+}\right)_{d}
$$

Then, if $\omega$ is a weight vector of degree $d$, then we put

$$
\mathscr{B}_{s}(\omega):=\mathscr{B}_{s}(d) \cap P_{s}(\omega), \mathscr{B}_{s}^{0}(\omega):=\mathscr{B}_{s}(\omega) \cap\left(P_{s}^{0}\right)_{d}, \mathscr{B}_{s}^{+}(\omega):=\mathscr{B}_{s}(\omega) \cap\left(P_{s}^{+}\right)_{d}
$$

Next, for a polynomial $f \in P_{s}$, we denote by $[f]$ the classes in $Q P_{s}$ represented by $f$. If $\omega$ is a weight vector and $f \in P_{s}(\omega)$, then denote by $[f]_{\omega}$ the classes in $Q P_{s}(\omega)$ represented by $f$. For a subset $\mathscr{B} \subset P_{s}$, we denote $[\mathscr{B}]=\{[f]: f \in \mathscr{B}\}$. If $\mathscr{B} \subset P_{s}(\omega)$, then we set $[\mathscr{B}]_{\omega}=\left\{[f]_{\omega}: f \in \mathscr{B}\right\}$. Thus, we see that $\left[\mathscr{B}_{s}(\omega)\right]_{\omega},\left[\mathscr{B}_{s}^{0}(\omega)\right]_{\omega}$ and $\left[\mathscr{B}_{s}^{+}(\omega)\right]_{\omega}$ are respectively the bases of the $\mathbb{F}_{2}$-vector spaces $Q P_{s}(\omega), Q P_{s}^{0}(\omega):=Q P_{s}(\omega) \cap\left(Q P_{s}^{0}\right)_{d}$ and $Q P_{s}^{+}(\omega):=Q P_{s}(\omega) \cap\left(Q P_{s}^{+}\right)_{d}$.

For a natural number $d$, let $\mu(d)=\min \left\{r \in \mathbb{N}: d=\sum_{1 \leqslant i \leqslant r}\left(2^{u_{i}}-1\right), u_{i}>0\right\}$. So, by Wood [55], $\left(Q P_{s}\right)_{d}=0$ if $\mu(d)>s$, and by Sum [50], $\mu(d)=r \leqslant s$ if and only if there exists uniquely a sequence of integers $u_{1}>u_{2}>\cdots>u_{r-1} \geqslant u_{r}>0$ such that $d=\sum_{1 \leqslant i \leqslant r}\left(2^{u_{i}}-1\right)$. From these, we only need to study $\left(Q P_{s}\right)_{d}$ in degrees $d$ satisfying $1 \leqslant \mu(d)=r \leqslant s$; moreover $d$ is of the form $r\left(2^{t}-1\right)+2^{t} . m$, whenever $t, m$ are non-negative integers such that $\mu(m)<r$. Indeed, if $\mu(d)=r=1$, then $d$ has the form $d=2^{u}-1=1 \cdot\left(2^{u}-1\right)+2^{u} \cdot 0=r \cdot\left(2^{u}-1\right)+2^{u} \cdot m$, where $u>0$ and $\mu(m=0)=0<1$. If $\mu(d)=r \geqslant 2$, then by Sum [50], $d$ has the form $\sum_{1 \leqslant i \leqslant r}\left(2^{u_{i}}-1\right)$, where $u_{1}>u_{2}>\cdots>u_{r-1} \geqslant u_{r}>0$. Consider $m=\sum_{1 \leqslant j \leqslant r-1}\left(2^{u_{j}-u_{r}}-1\right)$. According to Sum [50], we see that $\mu(m)=r-2<r$ if $u_{r-1}=u_{r}$ and $\mu(m)=r-1<r$ if $u_{r-1}>u_{r}$. Further, $d=r\left(2^{t}-1\right)+2^{t} . m$ with $t=u_{r}$.

The following homomorphisms will be used throughout the article. For $1 \leqslant i \leqslant s$, define the homomorphism $\rho_{i}: P_{s-1} \rightarrow P_{s}$ of algebras by substituting

$$
\rho_{i}\left(x_{j}\right)= \begin{cases}x_{j} & \text { if } 1 \leqslant j<i, \\ x_{j+1} & \text { if } i \leqslant j<s .\end{cases}
$$

The maps $\rho_{i}$ are also the monomorphism of $\mathscr{A}$-modules. We consider the set

$$
\mathcal{N}_{s}:=\left\{(i ; I) \mid I=\left(i_{1}, i_{2}, \ldots, i_{r}\right), 1 \leqslant i<i_{1}<i_{2}<\ldots<i_{r} \leqslant s, 0 \leqslant r<s\right\},
$$

where by convention, $I=\emptyset$, if $r=0$. Let $r=\ell(I)$ be the length of $I$.

Definition 2.2.6. Let $(i ; I) \in \mathcal{N}_{s}, r=\ell(I)$, and let $u$ be an integer with $1 \leqslant u \leqslant r$. A monomial $x=x_{1}^{a_{1}} x_{2}^{a_{2}} \ldots x_{s-1}^{a_{s-1}} \in P_{s-1}$ is said to be $u$-compatible with $(i ; I)$ if all of the following hold:

(i) $a_{i_{1}-1}=a_{i_{2}-1}=\ldots=a_{i_{(u-1)}-1}=2^{r}-1$,

(ii) $a_{i_{u}-1}>2^{r}-1$,

(iii) $\alpha_{r-t}\left(a_{i_{u}-1}\right)=1, \forall t, 1 \leqslant t \leqslant u$,

(iv) $\alpha_{r-t}\left(a_{i_{t}-1}\right)=1, \forall t, u<t \leqslant r$. 
Clearly, a monomial $x$ can be $u$-compatible with a given $(i ; I) \in \mathcal{N}_{s}$ for at most one value of $u$. By convention, $x$ is 1-compatible with $(i ; \emptyset)$.

Definition 2.2.7. Consider $(i ; I) \in \mathcal{N}_{s}, 0<r<s$ and put $x_{(I, u)}=x_{i_{u}}^{2^{r-1}+2^{r-2}+\cdots+2^{r-u}} \prod_{u<t \leqslant r} x_{i_{t}}^{2^{r-t}}$, for $1 \leqslant u \leqslant r, x_{(\emptyset, 1)}=1$. For a monomial $x \in P_{s-1}$, we define the monomial $\phi_{(i ; I)}(x)$ in $P_{s}$ by setting

$$
\phi_{(i ; I)}(x)= \begin{cases}\frac{x_{i}^{2^{r}-1} \rho_{i}(x)}{x_{(I, u)}} & \text { if there exist } u \text { such that } x \text { is } u \text {-compatible with }(i ; I), \\ 0 & \text { otherwise. }\end{cases}
$$

Then we have a linear transformation $\phi_{(i ; I)}: P_{s-1} \rightarrow P_{s}$. It is easily seen that $\phi_{(i ; \emptyset)}=\rho_{i}$ and that $\phi_{(i ; I)}$ is not an $\mathscr{A}$-homomorphism. Moreover, if $\phi_{(i ; I)}(x) \neq 0$, then $\omega\left(\phi_{(i ; I)}(x)\right)=\omega(x)$.

For a subset $U \subset P_{s-1}$, we denote by

$$
\begin{aligned}
& \Phi^{0}(U)=\bigcup_{1 \leqslant i \leqslant s} \phi_{(i ; \emptyset)}(U)=\bigcup_{1 \leqslant i \leqslant s} \rho_{i}(U), \\
& \Phi^{+}(U)=\bigcup_{(i ; I) \in \mathcal{N}_{s}, 0<\ell(I)<s} \phi_{(i ; I)}(U) \backslash P_{s}^{0} .
\end{aligned}
$$

Then, it is easy to see that if $U$ is a minimal set of generators for $\mathscr{A}$-module $P_{s-1}$ in degree $d$, then $\Phi^{0}(U)$ is also a minimal set of generators for $\mathscr{A}$-module $P_{s}^{0}$ in degree $d$.

Definition 2.2.8. For any $(i ; I) \in \mathcal{N}_{s}$, we define the homomorphism $p_{(i ; I)}: P_{s} \rightarrow P_{s-1}$ of $\mathbb{F}_{2^{-}}$ algebras by substituting

$$
p_{(i ; I)}\left(x_{j}\right)= \begin{cases}x_{j} & \text { if } 1 \leqslant j<i \\ \sum_{k \in I} x_{k-1} & \text { if } j=i \\ x_{j-1} & \text { if } i<j \leqslant s\end{cases}
$$

It should be noticed that $p_{(i ; I)}$ is also a homomorphism of $\mathscr{A}$-modules. In particular, we have $p_{(i ; \emptyset)}\left(x_{i}\right)=0$ for $1 \leqslant i \leqslant s$ and $p_{(i ; I)}\left(\rho_{i}(u)\right)=u$ for any $u \in P_{s-1}$. Moreover, the next technicality is important.

Lemma 2.2.9 (see [24]). If $x$ is a monomial in $P_{s}$, then $p_{(i ; I)}(x) \in P_{s-1}(\omega(x))$.

The lemma indicates that if $\omega$ is a weight vector and $x \in P_{s}(\omega)$, then $p_{(i ; I)}(x) \in P_{s-1}(\omega)$. Furthermore, $p_{(i ; I)}$ passes to a homomorphism from $Q P_{s}(\omega)$ to $Q P_{s-1}(\omega)$.

In Section 3, we use Lemma 2.2.9 and the results in Sum [47] to prove a certain subset of $Q P_{s}$ is linearly independent. More precisely, let $\mathscr{B}$ be a finite subset of $P_{s}$ consisting of some monomials of degree $d$. Denote by $|\mathscr{B}|$ the cardinal of $\mathscr{B}$. To prove the set $[\mathscr{B}]$ is linearly independent in $\left(Q P_{s}\right)_{d}$, we denote the elements of $\mathscr{B}$ by $b_{d, k}, 1 \leqslant k \leqslant n=|\mathscr{B}|$ and assume that there is a linear relation $\mathcal{S}=\sum_{k=1}^{n} \gamma_{k} b_{d, k} \equiv 0$, with $\gamma_{k} \in \mathbb{F}_{2}$ for all $k, 1 \leqslant k \leqslant n$. For $(i ; I) \in \mathcal{N}_{s}$, we explicitly compute $p_{(i ; I)}(\mathcal{S})$ in terms of the admissible monomials in $P_{s-1}\left(\bmod \left(\mathscr{A}^{+} \cdot P_{s-1}\right)\right)$. Computing from some relations $p_{(i ; I)}(\mathcal{S}) \equiv 0$ with $(i ; I) \in \mathcal{N}_{s}$, we obtain $\gamma_{k}=0$ for all $k$.

\section{3. $\mathscr{A}$-generators for $P_{5}$ in degree $3\left(2^{t}-1\right)+2^{t}$}

The goal of this section is to prove Theorem 1.1. More precisely, we explicitly determine all admissible monomials of degree $3\left(2^{t}-1\right)+2^{t}$ in $P_{5}$. We first recall a result of Singer [42] on the hit monomials in $P_{s}$.

\subsection{Singer's criterion on the hit monomials}

Definition 3.1.1. A monomial $z=x_{1}^{b_{1}} x_{2}^{b_{2}} \ldots x_{s}^{b_{s}}$ in $P_{s}$ is called a spike if $b_{i}=2^{t_{i}}-1$ for $t_{i}$ a non-negative integer and $i=1,2, \ldots, s$. If $z$ is a spike with $t_{1}>t_{2}>\ldots>t_{r-1} \geqslant t_{r}>0$ and $t_{j}=0$ for $j>r$, then it is called a minimal spike. 
Lemma 3.1.2. All the spikes in $P_{s}$ are admissible and their weight vectors are weakly decreasing. Furthermore, if a weight vector $\omega=\left(\omega_{1}, \omega_{2}, \ldots\right)$ is weakly decreasing and $\omega_{1} \leqslant s$, then there is a spike $\mathscr{Z}$ in $P_{s}$ such that $\omega(\mathscr{Z})=\omega$.

Proof. Let $\mathscr{Z}=\prod_{1 \leqslant i \leqslant s} x_{i}^{2^{d_{i}}-1}$ be a spike monomial in $P_{s}$. By way of contradiction, suppose that $\mathscr{Z}$ is inadmissible. Then, there exist monomials $y_{1}, y_{2}, \ldots, y_{m} \in P_{s}$ such that $y_{t}<\mathscr{Z}$ for all $t$ and $\mathscr{Z}=\sum_{1 \leqslant t \leqslant m} y_{t}+\sum_{j>0} S q^{j}\left(w_{j}\right)$, with suitable monomial $w_{j} \in P_{s}$. This implies that there is a monomial $u \in P_{s}$ with $\operatorname{deg} u<\operatorname{deg} \mathscr{Z}$ such that $S q^{n}(u)=\mathscr{Z}+f$, where $n>0$ and $f=\sum_{i} X_{i} \in$ $P_{s}, \operatorname{deg} X_{i}=\operatorname{deg} \mathscr{Z}$. Suppose $u=\prod_{1 \leqslant \ell \leqslant k} x_{\ell}^{a_{\ell}}$. Since $\operatorname{deg} u<\operatorname{deg} \mathscr{Z}$, ther is a number $t, 1 \leqslant t \leqslant s$ such that $a_{t}<2^{d_{t}}-1$. According to Cartan's formula, we have

$$
\mathscr{Z}+f=S q^{n}(u)=\sum_{i \geqslant 0} S q^{i}\left(x_{t}^{a_{t}}\right) S q^{n-i}\left(x_{1}^{a_{1}} \ldots x_{t-1}^{a_{t-1}} x_{t+1}^{a_{t+1}} \ldots x_{s}^{a_{s}}\right) .
$$

Since $2^{d_{t}}-1-a_{t}>0$ and $\mathscr{Z}$ is a term of $\sum_{i \geqslant 0} S q^{i}\left(x_{t}^{a_{t}}\right) S q^{n-i}\left(x_{1}^{a_{1}} \ldots x_{t-1}^{a_{t-1}} x_{t+1}^{a_{t+1}} \ldots x_{s}^{a_{s}}\right)$, hence $\mathscr{Z}$ is a term of $S q^{2^{d_{t}}-1-a_{t}}\left(x_{t}^{a_{t}}\right) S q^{n-\left(2^{d_{t}}-1-a_{t}\right)}\left(x_{1}^{a_{1}} \ldots x_{t-1}^{a_{t-1}} x_{t+1}^{a_{t+1}} \ldots x_{s}^{a_{s}}\right)$. On the other hand, $2^{d_{t}}-1-a_{t} \leqslant$ $2^{d_{t}}-1$, so there exist a number $\ell \geqslant 0$ such that $\alpha_{\ell}\left(2^{d_{t}}-1-a_{t}\right)=1$. This implies $\alpha_{\ell}\left(a_{t}\right)=0$. Since $\left(\begin{array}{c}a_{t} \\ 2^{d_{t}}-1-a_{t}\end{array}\right)$ is even, $S q^{2^{d_{t}}-1-a_{t}}\left(x_{t}^{a_{t}}\right)=0$. So, one gets $\mathscr{Z}=0$. This contradicts the fact that $\mathscr{Z}=\prod_{1 \leqslant i \leqslant s} x_{i}^{2^{d_{i}}-1} \neq 0$. Obviously, if $\mathscr{Z}$ is a spike, then its weight vector is weakly decreasing.

Now, assume that $\omega=\left(\omega_{1}, \omega_{2}, \ldots\right)$ is weakly decreasing and $\omega_{1} \leqslant s$. For $j \geqslant s$, we denote by $a_{j}$ the non-negative integer such that

$$
\alpha_{i}\left(a_{j}\right)= \begin{cases}1, & \text { if } \omega_{i+1} \geqslant j, \\ 0, & \text { if } \omega_{i+1}<j\end{cases}
$$

Denote by $d_{j}$ the greatest integer such that $\omega_{d_{j}} \geqslant j$. Since $\omega$ is weakly decreasing, $\alpha_{i}\left(a_{j}\right)=1$ for $1 \leqslant i<d_{j}$ and $\alpha_{i}\left(a_{j}\right)=0$ with $i \geqslant d_{j}$. So, $a_{j}=2^{d_{j}}-1$. On the other hand, we have $\omega_{i+1} \leqslant \omega_{1} \leqslant s$ for all $i \geqslant 0$. Hence, if $j>s$, then $a_{j}=0$. Therefore, $\mathscr{Z}=x_{1}^{a_{1}} x_{2}^{a_{2}} \ldots x_{s}^{a_{s}}$ is a spike in $P_{s}$ and $\omega(\mathscr{Z})=\omega$. The lemma is proved.

In [42], Singer pointed out that if $\mu(d) \leqslant s$, then there exists uniquely a minimal spike of degree $d$ in $P_{s}$. Further, we have the following.

Theorem 3.1.3 (see Singer [42]). Let $x$ be a monomial in $P_{s}$, where $\mu(\operatorname{deg}(x)) \leqslant s$. Suppose that $z$ is the minimal spike with $\operatorname{deg}(z)=\operatorname{deg}(x)$. Then, if $\omega(x)<\omega(z)$, then $x$ belongs to $\mathscr{A}^{+} \cdot P_{s}$.

\subsection{Proof of Theorem 1.1}

For convenience, we use the following notations:

$$
\begin{aligned}
& \mathbb{N}_{s}=\{1,2, \ldots, s\}, \\
& X_{\mathbb{I}}=X_{\left\{i_{1}, i_{2}, \ldots, i_{r}\right\}}=\prod_{i \in \mathbb{N}_{s} \backslash \mathbb{I}} x_{i}, \mathbb{I}=\left\{i_{1}, i_{2}, \ldots, i_{r}\right\} \subseteq \mathbb{N}_{s} .
\end{aligned}
$$

Specifically, $X_{\mathbb{N}_{s}}=1, X_{\emptyset}=x_{1} x_{2} \ldots x_{s}$, and $X_{\{i\}}=x_{1} \ldots \hat{x}_{i} \ldots x_{s}, i=1,2, \ldots, s$. Let $x=$ $x_{1}^{a_{1}} x_{2}^{a_{2}} \ldots x_{s}^{a_{s}} \in P_{s}$ and let $\mathbb{I}_{j}(x)=\left\{i \in \mathbb{N}_{s}: \alpha_{j}\left(a_{i}\right)=0\right\}$, for $j \geqslant 0$. Then, a careful but straightforward computation shows that $x=\prod_{j \geqslant 0} X_{\mathbb{I}_{j}(x)}^{2^{j}}$. Now, notice that Kameko's homomorphism $\widetilde{S q_{*}^{0}}=\left(\widetilde{S q_{*}^{0}}\right)_{\left(5,3\left(2^{t}-1\right)+2^{t}\right)}:\left(Q P_{5}\right)_{3\left(2^{t}-1\right)+2^{t}} \longrightarrow\left(Q P_{5}\right)_{2^{t+1}-4}$ is an epimorphism, and so, $\left(Q P_{5}\right)_{3\left(2^{t}-1\right)+2^{t}} \cong \operatorname{Ker}\left(\left(\widetilde{S q_{*}^{0}}\right)_{\left(5,3\left(2^{t}-1\right)+2^{t}\right)}\right) \bigoplus\left(Q P_{5}\right)_{2^{t+1}-4}$. Following our previous works [24, 25], the dimensions of $\left(Q P_{5}\right)_{2^{t+1}-4}$ are determined as follows. 
Theorem 3.2.1. Let $n=2^{t+1}-4$ with $t$ a positive integer. The dimension of the $\mathbb{F}_{2}$-vector space $\left(Q P_{5}\right)_{n}$ is determined by the following table:

\begin{tabular}{c|cccccc}
$n=2^{t+1}-4$ & $t=1$ & $t=2$ & $t=3$ & $t=4$ & $t=5$ & $t \geqslant 6$ \\
\hline $\operatorname{dim}\left(Q P_{5}\right)_{n}$ & 1 & 45 & 190 & 480 & 650 & 651
\end{tabular}

When $t=1$, we see that $2^{t+1}-4=0$ and $\left(Q P_{5}\right)_{0} \cong \mathbb{F}_{2}$. So, $\operatorname{dim}\left(\left(Q P_{5}\right)_{0}\right)=\operatorname{dim}\left(\mathbb{F}_{2}\right)=1$. We now determine $\operatorname{Ker}\left(\left(\widetilde{S q_{*}^{0}}\right)_{\left(5,3\left(2^{t}-1\right)+2^{t}\right)}\right)$. Put

$$
\omega_{(5, t)}=(3,3, \ldots, 3,1) \text {, and } \omega_{(5, t)}^{*}=(3,3, \ldots, 3)(t \text { times of } 3) .
$$

Then, an important key in the proof of the theorem is the following lemma.

Lemma 3.2.2. If $x$ is an admissible monomial of degree $3\left(2^{t}-1\right)+2^{t}$ in $P_{5}$ and $[x]$ belongs to $\operatorname{Ker}\left(\left(\widetilde{S q_{*}^{0}}\right)_{\left(5,3\left(2^{t}-1\right)+2^{t}\right)}\right)$ then $\omega(x)=\omega_{(5, t)}$.

Proof. We prove the lemma by induction on $t$. By Sum $[46,50]$, we see that the lemma holds for $t=1$.

Suppose $t>1$ and the lemma holds for $1,2, \ldots, t-1$. Observe that the monomial $z=$ $x_{1}^{2^{t+1}-1} x_{2}^{2^{t}-1} x_{3}^{2^{t}-1}$ is the minimal spike of degree $3\left(2^{t}-1\right)+2^{t}$ in $P_{5}$ and $\omega(z)=\omega_{(5, t)}$.

Since $3\left(2^{t}-1\right)+2^{t}$ is odd and $[x] \neq[0]$, one gets either $\omega_{1}(x)=3$ or $\omega_{1}(x)=5$. If $\omega_{1}(x)=5$, then $x=X_{\emptyset} y^{2}$ with $y$ a monomial of degree $2^{t+1}-4$ in $P_{5}$. Since $x$ is admissible, by Theorem 2.2.5, $y$ is admissible. Hence, $\left(\widetilde{S q}_{*}^{0}\right)_{\left(5,3\left(2^{t}-1\right)+2^{t}\right)}([x])=[y] \neq[0]$. This contradicts the face that $[x] \in \operatorname{Ker}\left(\left(\widetilde{S q_{*}^{0}}\right)_{\left(5,3\left(2^{t}-1\right)+2^{t}\right)}\right)$, so $\omega_{1}(x)=3$. Then, we have $x=X_{\{i, j\}} u^{2}$ with $1 \leqslant i<j \leqslant 5$ and $u$ an admissible monomial of degree $3\left(2^{t-1}-1\right)+2^{t-1}$ in $P_{5}$. Now, the lemma follows from the inductive hypothesis.

Following Lemma 3.2.2, it may be concluded that

$$
\operatorname{Ker}\left(\left(\widetilde{S q_{*}^{0}}\right)_{\left(5,3\left(2^{t}-1\right)+2^{t}\right)}\right)=\left(Q P_{5}^{0}\right)_{3\left(2^{t}-1\right)+2^{t}} \bigoplus \mathbb{V},
$$

where $\mathbb{V}=\left(\operatorname{Ker}\left(\left(\widetilde{S q_{*}^{0}}\right)_{\left(5,3\left(2^{t}-1\right)+2^{t}\right)}\right) \cap\left(Q P_{5}^{+}\right)_{3\left(2^{t}-1\right)+2^{t}}\right)$ is isomorphic to $Q P_{5}^{+}\left(\omega_{(5, t)}\right)$.

The case $\boldsymbol{t}=\mathbf{1}$. Following $[46,50]$, we have $\left|\mathscr{B}_{5}^{0}(5)\right|=\left|\mathscr{B}_{5}^{0}\left(\omega_{(5,1)}\right)\right|=45$ and $\left|\mathscr{B}_{5}^{+}\left(\omega_{(5,1)}\right)\right|=0$. This together with Theorem 3.2.1 imply that $\left(Q P_{5}\right)_{5}$ is 46 -dimensional.

The case $\boldsymbol{t}=2$. It should be noted that for each $s \geqslant 1$, Moetele and Mothebe indicated in [20] that $\operatorname{dim}\left(Q P_{s}\right)_{13}=\sum_{3 \leqslant j \leqslant 13}\left(\begin{array}{l}d \\ j\end{array}\right) C_{j}$, where $C_{j}, 3 \leqslant j \leqslant 13$, are determined by the following table:

\begin{tabular}{c|ccccccccccc}
$j$ & 3 & 4 & 5 & 6 & 7 & 8 & 9 & 10 & 11 & 12 & 13 \\
\hline$C_{j}$ & 3 & 23 & 105 & 268 & 415 & 438 & 322 & 164 & 55 & 11 & 1
\end{tabular}

This implies that $\left(Q P_{5}\right)_{13}$ has dimension 250. Their methods are based on Kameko's homomorphism [12] and a result on the admissible monomials [20, Theorem 2.7]. For this case, we will prove it in another way. More precisely, to compute $\left(Q P_{5}\right)_{13}$, we determine explicitly all the admissible monomials of degree 13 in $P_{5}$ by using Lemma 2.2.9 and some homomorphisms in Subsection 2.2. Detailed calculations are performed as follows. By Sum [47], we have $\left|\mathscr{B}_{4}(13)\right|=\left|\mathscr{B}_{4}\left(\omega_{(5,2)}\right)\right|=35$. A simple computation shows that $\mathscr{B}_{5}^{0}(13)=\mathscr{B}_{5}^{0}\left(\omega_{(5,2)}\right)=\Phi^{0}\left(\mathscr{B}_{4}(13)\right)$ is the set consisting of 145 admissible monomials, namely $q_{2, k}$, for $1 \leqslant k \leqslant 145$ (see Subsection 5.1), i.e., $\left(Q P_{5}^{0}\right)_{13}$ is 145-dimensional. Next, we need to calculate $Q P_{5}^{+}\left(\omega_{(5,2)}\right)$. It is not difficult to show that $\Phi^{+}\left(\mathscr{B}_{4}\left(\omega_{(5,2)}\right)\right) \cup\left\{x_{1} x_{2}^{2} x_{3}^{4} x_{4}^{3} x_{5}^{3}, \quad x_{1}^{3} x_{2}^{4} x_{3}^{3} x_{4} x_{5}^{2}, \quad x_{1}^{3} x_{2}^{4} x_{3} x_{4}^{3} x_{5}^{2}, \quad x_{1}^{3} x_{2}^{4} x_{3} x_{4}^{2} x_{5}^{3}\right\}$ is the set consisting of 60 admissible monomials, namely $b_{13, k}$, for $1 \leqslant k \leqslant 60$ (see Subsection 5.1). The following assertion is of key importance in the proof.

Proposition 3.2.3. The set $\left\{\left[b_{13, k}\right]_{\omega_{(5,2)}}, 1 \leqslant k \leqslant 60\right\}$ is a basis of $\mathbb{F}_{2}$-vector space $Q P_{5}^{+}\left(\omega_{(5,2)}\right)$.

We need some lemmas for the proof of the proposition. It is rather straightforward to prove the following directly, so we omit the details by leaving them to the interested reader for a direct check. 
Lemma 3.2.4. The following monomials are strictly inadmissible:

(i) $x_{i}^{2} x_{j}^{2} x_{k} x_{\ell} x_{m}^{3}, x_{i}^{2} x_{j} x_{k}^{2} x_{\ell} x_{m}^{3}, x_{i}^{2} x_{j} x_{k} x_{\ell}^{2} x_{m}^{3}, i<j<k<\ell$. Here $(i, j, k, \ell, m)$ is a permutation of $(1,2,3,4,5)$.

(ii) $\rho_{i}(u), 1 \leqslant i \leqslant 5$, where $u$ is one of the following monomials:

$$
x_{1}^{2} x_{2} x_{3}^{3} x_{4}^{3}, x_{1}^{2} x_{2}^{3} x_{3} x_{4}^{3}, x_{1}^{2} x_{2}^{3} x_{3}^{3} x_{4}, x_{1}^{3} x_{2}^{2} x_{3} x_{4}^{3}, x_{1}^{3} x_{2}^{2} x_{3}^{3} x_{4}, x_{1}^{3} x_{2}^{3} x_{3}^{2} x_{4}, x_{1}^{3} x_{2}^{4} x_{3}^{3} x_{4}^{3} .
$$

The next lemma is important.

Lemma 3.2.5. The following monomials are strictly inadmissible:

$$
\begin{array}{lllll}
x_{1} x_{2}^{2} x_{3}^{2} x_{4} x_{5}^{7}, & x_{1} x_{2}^{2} x_{3}^{2} x_{4}^{3} x_{5}^{5}, & x_{1} x_{2}^{2} x_{3}^{2} x_{4}^{5} x_{5}^{3}, & x_{1} x_{2}^{2} x_{3}^{2} x_{4}^{7} x_{5}, & x_{1} x_{2}^{2} x_{3}^{3} x_{4}^{2} x_{5}^{5}, \\
x_{1} x_{2}^{2} x_{3}^{3} x_{4}^{6} x_{5}, & x_{1} x_{2}^{2} x_{3}^{6} x_{4} x_{5}^{3}, & x_{1} x_{2}^{2} x_{3}^{6} x_{4}^{3} x_{5}, & x_{1} x_{2}^{2} x_{3}^{7} x_{4}^{2} x_{5}, & x_{1} x_{2}^{3} x_{3}^{2} x_{4}^{2} x_{5}^{5}, \\
x_{1} x_{2}^{3} x_{3}^{2} x_{4}^{6} x_{5}, & x_{1} x_{2}^{3} x_{3}^{6} x_{4}^{2} x_{5}, & x_{1} x_{2}^{6} x_{3}^{2} x_{4} x_{5}^{3}, & x_{1} x_{2}^{6} x_{3}^{2} x_{4}^{3} x_{5}, & x_{1} x_{2}^{6} x_{3}^{3} x_{4}^{2} x_{5}, \\
x_{1} x_{2}^{7} x_{3}^{2} x_{4}^{2} x_{5}, & x_{1}^{3} x_{2} x_{3}^{2} x_{4}^{2} x_{5}^{5}, & x_{1}^{3} x_{2} x_{3}^{2} x_{4}^{6} x_{5}, & x_{1}^{3} x_{2} x_{3}^{6} x_{4}^{2} x_{5}, & x_{1}^{3} x_{2}^{5} x_{3}^{2} x_{4}^{2} x_{5}, \\
x_{1}^{7} x_{2} x_{3}^{2} x_{4}^{2} x_{5} . & & &
\end{array}
$$

Proof. We prove the lemma for $x=x_{1} x_{2}^{2} x_{3}^{2} x_{4}^{3} x_{5}^{5}$. The others can be proven by a similar computation. By a direct computation using the Cartan formula, we have

$$
\begin{aligned}
x= & x_{1} x_{2} x_{3}^{2} x_{4}^{3} x_{5}^{6}+x_{1} x_{2}^{2} x_{3} x_{4}^{3} x_{5}^{6}+S q^{1}\left(x_{1} x_{2} x_{3}^{2} x_{4}^{3} x_{5}^{5}+x_{1} x_{2} x_{3}^{2} x_{4}^{5} x_{5}^{3}+x_{1} x_{2} x_{3}^{4} x_{4}^{3} x_{5}^{3}+x_{1} x_{2}^{2} x_{3} x_{4}^{3} x_{5}^{5}\right. \\
& \left.+x_{1} x_{2}^{2} x_{3} x_{4}^{5} x_{5}^{3}+x_{1} x_{2}^{4} x_{3} x_{4}^{3} x_{5}^{3} .+x_{1}^{2} x_{2} x_{3} x_{4}^{5} x_{5}^{3}+x_{1}^{4} x_{2} x_{3} x_{4}^{3} x_{5}^{3}\right) \\
& +S q^{2}\left(x_{1} x_{2} x_{3} x_{4}^{3} x_{5}^{5}+x_{1} x_{2}^{2} x_{3}^{2} x_{4}^{3} x_{5}^{3}+x_{1}^{2} x_{2} x_{3}^{2} x_{4}^{3} x_{5}^{3}+x_{1}^{2} x_{2}^{2} x_{3} x_{4}^{3} x_{5}^{3}\right) \\
& +S q^{4}\left(x_{1} x_{2} x_{3} x_{4}^{3} x_{5}^{3}\right) \bmod \left(\mathrm{P}_{5}^{-}\left(\omega_{(5,2)}\right)\right) .
\end{aligned}
$$

This equality implies that $x$ is strictly inadmissible.

Proof of Proposition 3.2.3. Let $x$ be an admissible monomial of degree 13 in $P_{5}$ and $\omega(x)=\omega_{(5,2)}$. Then, $x=X_{\{i, j\}} u^{2}$ with $1 \leqslant i<j \leqslant 5$ and $u$ a monomial of degree 5 in $P_{5}$. Since $x$ is admissible, according to Theorem 2.2.5, we get $u \in \mathscr{B}_{5}\left(\omega_{(5,1)}\right)$.

By a direct computation, we see that for all $y \in \mathscr{B}_{5}\left(\omega_{(5,1)}\right)$, such that $X_{\{i, j\}} y^{2} \neq b_{13, k}, \forall k, 1 \leqslant$ $k \leqslant 60$, there is a monomial $w$ which is given in one of Lemmas 3.2.4, and 3.2.5 such that $X_{\{i, j\}} y^{2}=$ $w z^{2^{r}}$ with a monomial $z \in P_{5}$ and $r=\max \left\{m \in \mathbb{Z}: \omega_{m}(w)>0\right\}$. By Theorem 2.2.5, $X_{\{i, j\}} y^{2}$ is inadmissible. Since $x=X_{\{i, j\}} u^{2}$ is admissible, one gets $x=b_{13, k}$ for suitable $k$.

We now prove the set $\left\{\left[b_{13, k}\right]_{\omega_{(5,2)}}: 1 \leqslant k \leqslant 60\right\}$ is linearly independent in $\left(Q P_{5}\right)_{13}$. Suppose there is a linear relation

$$
\mathcal{S}=\sum_{1 \leqslant k \leqslant 60} \gamma_{k} b_{13, k} \equiv_{\omega_{(5,2)}} 0,
$$

where $\gamma_{k} \in \mathbb{F}_{2}$.

From a result in [47], $\operatorname{dim} Q P_{4}^{+}\left(\omega_{(5,2)}\right)=23$, with the basis $\left\{\left[u_{i}\right]_{\omega_{(5,2)}}: 1 \leqslant i \leqslant 23\right\}$, where

$$
\begin{array}{lllll}
u_{1} \cdot x_{1} x_{2}^{2} x_{3}^{3} x_{4}^{7}, & u_{2} \cdot x_{1} x_{2}^{2} x_{3}^{7} x_{4}^{3}, & u_{3} \cdot x_{1} x_{2}^{3} x_{3}^{2} x_{4}^{7}, & u_{4} \cdot x_{1} x_{2}^{3} x_{3}^{3} x_{4}^{6}, & u_{5} \cdot x_{1} x_{2}^{3} x_{3}^{6} x_{4}^{3}, \\
u_{6} \cdot x_{1} x_{2}^{3} x_{3}^{7} x_{4}^{2}, & u_{7} \cdot x_{1} x_{2}^{6} x_{3}^{3} x_{4}^{3}, & u_{8} \cdot x_{1} x_{2}^{7} x_{3}^{2} x_{4}^{3}, & u_{9} \cdot x_{1} x_{2}^{7} x_{3}^{3} x_{4}^{2}, & u_{10} \cdot x_{1}^{3} x_{2} x_{3}^{2} x_{4}^{7}, \\
u_{11} \cdot x_{1}^{3} x_{2} x_{3}^{3} x_{4}^{6}, & u_{12} \cdot x_{1}^{3} x_{2} x_{3}^{6} x_{4}^{3}, & u_{13} \cdot x_{1}^{3} x_{2} x_{3}^{7} x_{4}^{2}, & u_{14} \cdot x_{1}^{3} x_{2}^{3} x_{3} x_{4}^{6}, & u_{15} \cdot x_{1}^{3} x_{2}^{3} x_{3}^{3} x_{4}^{4}, \\
u_{16} \cdot x_{1}^{3} x_{2}^{3} x_{3}^{4} x_{4}^{3}, & u_{17} \cdot x_{1}^{3} x_{2}^{3} x_{3}^{5} x_{4}^{2}, & u_{18} \cdot x_{1}^{3} x_{2}^{5} x_{3}^{2} x_{4}^{3}, & u_{19} \cdot x_{1}^{3} x_{2}^{5} x_{3}^{3} x_{4}^{2}, & u_{20} \cdot x_{1}^{3} x_{2}^{7} x_{3} x_{4}^{2}, \\
u_{21} \cdot x_{1}^{7} x_{2} x_{3}^{2} x_{4}^{3}, & u_{22} \cdot x_{1}^{7} x_{2} x_{3}^{3} x_{4}^{2}, & u_{23} \cdot x_{1}^{7} x_{2}^{3} x_{3} x_{4}^{2} . & &
\end{array}
$$

We explicitly compute $p_{(i ; I)}(\mathcal{S})$ in terms $u_{j}, 1 \leqslant j \leqslant 23$. By a direct computation using Lemma 2.2 .9 , Theorem 3.1.3, and from the relations $p_{(i ; j)}(\mathcal{S}) \equiv_{\omega_{(5,2)}} 0$ with either $i=1, j=2,3$ or $i=2, j=$ 3,4 , one gets

$$
\left\{\begin{array}{l}
\gamma_{k}=0, k \in \mathbb{J} \\
\gamma_{27}=\gamma_{55}, \gamma_{14}=\gamma_{49}=\gamma_{58}, \gamma_{13}=\gamma_{20}=\gamma_{43}=\gamma_{59} \\
\gamma_{30}+\gamma_{35}+\gamma_{58}=0, \gamma_{44}+\gamma_{55}+\gamma_{56}+\gamma_{58}=\gamma_{47}+\gamma_{53}+\gamma_{54}+\gamma_{59}=0
\end{array}\right.
$$


Here $\mathbb{J}=\{1,2,3,4,5,6,7,8,9,10,11,12,15,16,17,18,19,21,22,23,24,25,26,28,29$, $31,38,39,40,41,42,45,46,48,50,51,57,60\}$. Then, the relation (3.2.1) becomes

$$
\mathcal{S}=\sum_{k \in \mathbb{N}_{60} \backslash \mathbb{J}} \gamma_{k} b_{13, k} \equiv_{\omega_{(5,2)}} 0 .
$$

Applying the homomorphisms $p_{(1 ; 4)}, p_{(3 ; 4)}, p_{(3 ; 5)}, p_{(4 ; 5)}: P_{5} \rightarrow P_{4}$ to $(3.2 .2)$, we obtain $\gamma_{k}=$ $0,1 \leqslant k \leqslant 60$. This finishes the proof.

Since $\operatorname{dim}\left(Q P_{5}^{0}\right)_{13}=145$, from Theorem 3.2.1 and Proposition 3.2.3, one gets $\operatorname{dim}\left(Q P_{5}\right)_{13}=250$. For $t \geqslant 3$, we have $\left|\mathscr{B}_{4}\left(3\left(2^{t}-1\right)+2^{t}\right)\right|=45$ (see Sum [47]). By a simple computation, we get

$$
\mathscr{B}_{5}^{0}\left(3\left(2^{t}-1\right)+2^{t}\right)=\mathscr{B}_{5}^{0}\left(\omega_{(5, t)}\right)=\Phi^{0}\left(\mathscr{B}_{4}\left(3\left(2^{t}-1\right)+2^{t}\right)\right)=\left\{q_{t, k}: 1 \leqslant k \leqslant 195\right\},
$$

for all $t \geqslant 3$. Here, the monomials $q_{t, k}, 1 \leqslant k \leqslant 195$, are listed in Subsection 5.1.

We now compute $Q P_{5}^{+}\left(\omega_{(5, t)}\right)$ for $t \geqslant 3$.

The case $\boldsymbol{t}=3$. We obtain the following.

Proposition 3.2.6. $Q P_{5}^{+}\left(\omega_{(5,3)}\right)$ is the $\mathbb{F}_{2}$-vector space of dimension 260 with a basis consisting of all the classes represented by the monomials $b_{29, k}, 1 \leqslant k \leqslant 260$, which are determined as in Subsection 5.2.

To prove the proposition, we need some results. The following is a corollary of a result in [47].

Lemma 3.2.7. If $\bar{x}$ is one of the following monomials then $\rho_{i}(\bar{x}), 1 \leqslant i \leqslant 5$ are strictly inadmissible:

$$
\begin{array}{llllll}
x_{1}^{6} x_{2} x_{3}^{7} x_{4}^{7}, & x_{1}^{6} x_{2}^{7} x_{3} x_{4}^{7}, & x_{1}^{6} x_{2}^{7} x_{3}^{7} x_{4}, & x_{1}^{7} x_{2}^{6} x_{3} x_{4}^{7}, & x_{1}^{7} x_{2}^{6} x_{3}^{7} x_{4}, & x_{1}^{7} x_{2}^{7} x_{3}^{6} x_{4}, \\
x_{1}^{2} x_{2}^{5} x_{3}^{7} x_{4}^{7}, & x_{1}^{2} x_{2}^{7} x_{3}^{5} x_{4}^{7}, & x_{1}^{2} x_{2}^{7} x_{3}^{7} x_{4}^{5}, & x_{1}^{7} x_{2}^{2} x_{3}^{5} x_{4}^{7}, & x_{1}^{7} x_{2}^{2} x_{3}^{7} x_{4}^{5}, & x_{1}^{7} x_{2}^{7} x_{3}^{2} x_{4}^{5}, \\
x_{1}^{3} x_{2}^{4} x_{3}^{7} x_{4}^{7}, & x_{1}^{3} x_{2}^{7} x_{3}^{4} x_{4}^{7}, & x_{1}^{3} x_{2}^{7} x_{3}^{7} x_{4}^{4}, & x_{1}^{7} x_{2}^{3} x_{3}^{4} x_{4}^{7}, & x_{1}^{7} x_{2}^{3} x_{3}^{7} x_{4}^{4}, & x_{1}^{7} x_{2}^{7} x_{3}^{3} x_{4}^{4}, \\
x_{1}^{3} x_{2}^{6} x_{3}^{5} x_{4}^{7}, & x_{1}^{3} x_{2}^{6} x_{3}^{7} x_{4}^{5}, & x_{1}^{3} x_{2}^{7} x_{3}^{6} x_{4}^{5}, & x_{1}^{6} x_{2}^{3} x_{3}^{5} x_{4}^{7}, & x_{1}^{6} x_{2}^{3} x_{3}^{7} x_{4}^{5}, & x_{1}^{6} x_{2}^{7} x_{3}^{3} x_{4}^{5}, \\
x_{1}^{7} x_{2}^{3} x_{3}^{6} x_{4}^{5}, & x_{1}^{7} x_{2}^{6} x_{3}^{3} x_{4}^{5}, & x_{1}^{7} x_{2}^{7} x_{3}^{8} x_{4}^{7} . & &
\end{array}
$$

Lemma 3.2.8. The following monomials are strictly inadmissible:

(i) $x_{k}^{2} x_{\ell} x_{m}^{5} x_{t}^{6} x_{u}^{7}, k<\ell, x_{k}^{2} x_{\ell} x_{m}^{4} x_{t}^{7} x_{u}^{7}, x_{k}^{2} x_{\ell}^{4} x_{m} x_{t}^{7} x_{u}^{7}, x_{k}^{6} x_{\ell} x_{m}^{2} x_{t}^{5} x_{u}^{7}, x_{k}^{6} x_{\ell}^{3} x_{m}^{4} x_{t} x_{u}^{7}, k<\ell<m$. Here $(k, \ell, m, t, u)$ is a permutation of $(1,2,3,4,5)$.

(ii) $x_{1} x_{2}^{3} x_{3}^{6} x_{4}^{6} x_{5}^{5}, \quad x_{1} x_{2}^{6} x_{3}^{3} x_{4}^{6} x_{5}^{5}, \quad x_{1} x_{2}^{6} x_{3}^{6} x_{4}^{3} x_{5}^{5}, \quad x_{1}^{3} x_{2} x_{3}^{6} x_{4}^{6} x_{5}^{5}$, $x_{1}^{3} x_{2}^{5} x_{3}^{6} x_{4}^{6} x_{5}, \quad x_{1}^{3} x_{2}^{6} x_{3} x_{4}^{5} x_{5}^{6}, \quad x_{1}^{3} x_{2}^{6} x_{3} x_{4}^{6} x_{5}^{5}, \quad x_{1}^{3} x_{2}^{6} x_{3}^{5} x_{4} x_{5}^{6}$, $x_{1}^{3} x_{2}^{6} x_{3}^{5} x_{4}^{6} x_{5}, \quad x_{1}^{3} x_{2}^{6} x_{3}^{6} x_{4} x_{5}^{5}, \quad x_{1}^{3} x_{2}^{6} x_{3}^{6} x_{4}^{5} x_{5}$.

Proof. The proof of the lemma is straightforward.

Lemma 3.2.9. If $(i, j, k, \ell, m)$ is a permutation of $(1,2,3,4,5)$ such that $i<j<k<\ell$ then the following monomials are strictly inadmissible:

$$
\begin{array}{lllll}
x_{i} x_{j}^{6} x_{k}^{6} x_{\ell} x_{m}^{7}, & x_{i}^{6} x_{j} x_{k} x_{\ell}^{6} x_{m}^{7}, & x_{i}^{6} x_{j} x_{k}^{6} x_{\ell} x_{m}^{7}, & x_{i}^{6} x_{j}^{6} x_{k} x_{\ell} x_{m}^{7}, & x_{i} x_{j}^{2} x_{k}^{6} x_{\ell}^{5} x_{m}^{7}, \\
x_{i} x_{j}^{6} x_{k}^{2} x_{\ell}^{5} x_{m}^{7}, & x_{i}^{3} x_{j}^{4} x_{k} x_{\ell}^{6} x_{m}^{7}, & x_{i}^{3} x_{j}^{4} x_{k}^{6} x_{\ell} x_{m}^{7}, & x_{i}^{3} x_{j}^{6} x_{k}^{4} x_{\ell} x_{m}^{7}, & x_{i}^{3} x_{j}^{6} x_{k} x_{\ell}^{4} x_{m}^{7}, \\
x_{i}^{6} x_{j} x_{k}^{3} x_{\ell}^{5} x_{m}^{6}, & x_{i}^{6} x_{j}^{3} x_{k} x_{\ell}^{5} x_{m}^{6}, & x_{i}^{6} x_{j}^{3} x_{k}^{5} x_{\ell} x_{m}^{6}, & x_{i}^{2} x_{j}^{2} x_{k}^{5} x_{\ell}^{5} x_{m}^{7}, & x_{i}^{2} x_{j}^{5} x_{k}^{2} x_{\ell}^{5} x_{m}^{7}, \\
x_{i}^{3} x_{j}^{4} x_{k}^{3} x_{\ell}^{4} x_{m}^{7}, & x_{i}^{2} x_{j}^{3} x_{k}^{4} x_{\ell}^{5} x_{m}^{7}, & x_{i}^{2} x_{j}^{3} x_{k}^{5} x_{\ell}^{4} x_{m}^{7}, & x_{i}^{2} x_{j}^{4} x_{k}^{3} x_{\ell}^{5} x_{m}^{7}, & x_{i}^{2} x_{j}^{5} x_{k}^{3} x_{\ell}^{4} x_{m}^{7}, \\
x_{i}^{3} x_{j}^{2} x_{k}^{4} x_{\ell}^{5} x_{m}^{7}, & x_{i}^{3} x_{j}^{2} x_{k}^{5} x_{\ell}^{4} x_{m}^{7}, & x_{i}^{3} x_{j}^{4} x_{k}^{2} x_{\ell}^{5} x_{m}^{7}, & x_{i}^{2} x_{j}^{3} x_{k}^{5} x_{\ell}^{5} x_{m}^{6}, & x_{i}^{2} x_{j}^{5} x_{k}^{3} x_{\ell}^{5} x_{m}^{6}, \\
x_{i}^{3} x_{j}^{2} x_{k}^{5} x_{\ell}^{5} x_{m}^{6}, & x_{i}^{3} x_{j}^{5} x_{k}^{2} x_{\ell}^{5} x_{m}^{6} . & x_{i}^{3} x_{j}^{3} x_{k}^{4} x_{\ell}^{5} x_{m}^{6}, & x_{i}^{3} x_{j}^{3} x_{k}^{5} x_{\ell}^{4} x_{m}^{6}, & x_{i}^{3} x_{j}^{4} x_{k}^{3} x_{\ell}^{5} x_{m}^{6}, \\
x_{i}^{3} x_{j}^{5} x_{k}^{3} x_{\ell}^{4} x_{m}^{6} . & & &
\end{array}
$$


Proof. We prove the lemma for the monomial $x_{i} x_{j}^{6} x_{k}^{6} x_{\ell} x_{m}^{7}$. The others can be proved by a similar computation. By using the Cartan formula, we have

$$
\begin{aligned}
x_{i} x_{j}^{6} x_{k}^{6} x_{\ell} x_{m}^{7}= & S q^{1}\left(x_{i}^{2} x_{j}^{5} x_{k}^{5} x_{\ell} x_{m}^{7}\right)+S q^{2}\left(x_{i} x_{j}^{5} x_{k}^{5} x_{\ell} x_{m}^{7}+x_{i} x_{j}^{3} x_{k}^{5} x_{\ell} x_{m}^{9}\right) \\
& +x_{i} x_{j}^{6} x_{k}^{5} x_{\ell}^{2} x_{m}^{7}+x_{i} x_{j}^{5} x_{k}^{6} x_{\ell}^{2} x_{m}^{7} \bmod \left(P_{5}^{-}\left(\omega_{(5,3)}^{*}\right)\right) .
\end{aligned}
$$

This relation implies that $x_{i} x_{j}^{6} x_{k}^{6} x_{\ell} x_{m}^{7}$ is strictly inadmissible. The lemma is proven.

Lemma 3.2.10. The following monomials are strictly inadmissible:

$x_{1} x_{2}^{6} x_{3}^{7} x_{4}^{8} x_{5}^{7}, \quad x_{1} x_{2}^{7} x_{3}^{6} x_{4}^{8} x_{5}^{7}, \quad x_{1} x_{2}^{7} x_{3}^{10} x_{4}^{4} x_{5}^{7}, \quad x_{1} x_{2}^{7} x_{3}^{10} x_{4}^{7} x_{5}^{4}, \quad x_{1}^{3} x_{2}^{3} x_{3}^{4} x_{4}^{12} x_{5}^{7}$, $x_{1}^{3} x_{2}^{3} x_{3}^{12} x_{4}^{4} x_{5}^{7}, \quad x_{1}^{3} x_{2}^{3} x_{3}^{12} x_{4}^{7} x_{5}^{4}, \quad x_{1}^{3} x_{2}^{5} x_{3}^{6} x_{4}^{3} x_{5}^{12}, \quad x_{1}^{3} x_{2}^{5} x_{3}^{6} x_{4}^{11} x_{5}^{4}, \quad x_{1}^{3} x_{2}^{5} x_{3}^{8} x_{4}^{6} x_{5}^{7}$, $x_{1}^{3} x_{2}^{5} x_{3}^{8} x_{4}^{7} x_{5}^{6}, \quad x_{1}^{3} x_{2}^{5} x_{3}^{9} x_{4}^{6} x_{5}^{6}, \quad x_{1}^{3} x_{2}^{5} x_{3}^{14} x_{4}^{3} x_{5}^{4}, \quad x_{1}^{3} x_{2}^{12} x_{3}^{3} x_{4}^{5} x_{5}^{6}, \quad x_{1}^{3} x_{2}^{13} x_{3}^{6} x_{4}^{3} x_{5}^{4}$, $x_{1}^{7} x_{2} x_{3}^{6} x_{4}^{8} x_{5}^{7}, \quad x_{1}^{7} x_{2} x_{3}^{10} x_{4}^{4} x_{5}^{7}, \quad x_{1}^{7} x_{2} x_{3}^{10} x_{4}^{7} x_{5}^{4}, \quad x_{1}^{7} x_{2}^{8} x_{3}^{3} x_{4}^{5} x_{5}^{6}, \quad x_{1}^{7} x_{2}^{9} x_{3}^{2} x_{4}^{4} x_{5}^{7}$, $x_{1}^{7} x_{2}^{9} x_{3}^{2} x_{4}^{7} x_{5}^{4}, \quad x_{1}^{7} x_{2}^{9} x_{3}^{7} x_{4}^{2} x_{5}^{4}$.

Proof. We prove the lemma for the monomial $x=x_{1}^{3} x_{2}^{5} x_{3}^{6} x_{4}^{3} x_{5}^{12}$. The others can be proved by a similar computation. By a direct computation, we have

$$
\begin{aligned}
& x=x_{1}^{2} x_{2}^{3} x_{3}^{5} x_{4}^{6} x_{5}^{13}+x_{1}^{2} x_{2}^{3} x_{3}^{5} x_{4}^{12} x_{5}^{7}+x_{1}^{2} x_{2}^{5} x_{3}^{5} x_{4}^{6} x_{5}^{11}+x_{1}^{2} x_{2}^{5} x_{3}^{5} x_{4}^{10} x_{5}^{7}+x_{1}^{2} x_{2}^{5} x_{3}^{9} x_{4}^{6} x_{5}^{7} \\
& +x_{1}^{2} x_{2}^{11} x_{3}^{4} x_{4}^{5} x_{5}^{7}+x_{1}^{2} x_{2}^{11} x_{3}^{5} x_{4}^{4} x_{5}^{7}+x_{1}^{2} x_{2}^{13} x_{3}^{3} x_{4}^{4} x_{5}^{7}+x_{1}^{2} x_{2}^{13} x_{3}^{4} x_{4}^{3} x_{5}^{7}+x_{1}^{3} x_{2}^{3} x_{3}^{4} x_{4}^{6} x_{5}^{13} \\
& +x_{1}^{3} x_{2}^{3} x_{3}^{4} x_{4}^{12} x_{5}^{7}+x_{1}^{3} x_{2}^{3} x_{3}^{5} x_{4}^{6} x_{5}^{12}+x_{1}^{3} x_{2}^{3} x_{3}^{6} x_{4}^{5} x_{5}^{12}+x_{1}^{3} x_{2}^{3} x_{3}^{12} x_{4}^{4} x_{5}^{7}+x_{1}^{3} x_{2}^{4} x_{3}^{3} x_{4}^{6} x_{5}^{13} \\
& +x_{1}^{3} x_{2}^{4} x_{3}^{3} x_{4}^{12} x_{5}^{7}+x_{1}^{3} x_{2}^{4} x_{3}^{5} x_{4}^{6} x_{5}^{11}+x_{1}^{3} x_{2}^{4} x_{3}^{5} x_{4}^{10} x_{5}^{7}+x_{1}^{3} x_{2}^{4} x_{3}^{6} x_{4}^{3} x_{5}^{13}+x_{1}^{3} x_{2}^{4} x_{3}^{6} x_{4}^{9} x_{5}^{7} \\
& +x_{1}^{3} x_{2}^{4} x_{3}^{12} x_{4}^{3} x_{5}^{7}+x_{1}^{3} x_{2}^{5} x_{3}^{2} x_{4}^{5} x_{5}^{14}+x_{1}^{3} x_{2}^{5} x_{3}^{2} x_{4}^{6} x_{5}^{13}+x_{1}^{3} x_{2}^{5} x_{3}^{3} x_{4}^{4} x_{5}^{14}+x_{1}^{3} x_{2}^{5} x_{3}^{5} x_{4}^{6} x_{5}^{10} \\
& +x_{1}^{3} x_{2}^{5} x_{3}^{6} x_{4}^{2} x_{5}^{13}+x_{1}^{5} x_{2}^{5} x_{3}^{5} x_{4}^{5} x_{5}^{9}+S q^{1}\left(h_{1}\right)+S q^{2}\left(h_{2}\right)+S q^{4}\left(h_{4}\right) \bmod \left(P_{5}^{-}\left(\omega_{(5,3)}\right)\right) \text {, where } \\
& h_{1}=x_{1}^{3} x_{2}^{3} x_{3}^{5} x_{4}^{6} x_{5}^{11}+x_{1}^{3} x_{2}^{3} x_{3}^{5} x_{4}^{10} x_{5}^{7}+x_{1}^{3} x_{2}^{3} x_{3}^{9} x_{4}^{2} x_{5}^{11}+x_{1}^{3} x_{2}^{3} x_{3}^{9} x_{4}^{3} x_{5}^{10}+x_{1}^{3} x_{2}^{3} x_{3}^{9} x_{4}^{6} x_{5}^{7} \\
& +x_{1}^{3} x_{2}^{5} x_{3}^{2} x_{4}^{5} x_{5}^{13}+x_{1}^{3} x_{2}^{5} x_{3}^{3} x_{4}^{4} x_{5}^{13}+x_{1}^{3} x_{2}^{5} x_{3}^{4} x_{4}^{5} x_{5}^{11}+x_{1}^{3} x_{2}^{5} x_{3}^{4} x_{4}^{9} x_{5}^{7}+x_{1}^{3} x_{2}^{5} x_{3}^{9} x_{4}^{4} x_{5}^{7} \\
& +x_{1}^{3} x_{2}^{6} x_{3}^{5} x_{4}^{5} x_{5}^{9}+x_{1}^{3} x_{2}^{9} x_{3}^{2} x_{4}^{3} x_{5}^{11}+x_{1}^{3} x_{2}^{11} x_{3}^{3} x_{4}^{4} x_{5}^{7}+x_{1}^{3} x_{2}^{11} x_{3}^{4} x_{4}^{3} x_{5}^{7}+x_{1}^{10} x_{2}^{3} x_{3}^{3} x_{4}^{3} x_{5}^{9} \\
& +x_{1}^{10} x_{2}^{3} x_{3}^{3} x_{4}^{5} x_{5}^{7}+x_{1}^{10} x_{2}^{3} x_{3}^{5} x_{4}^{3} x_{5}^{7}+x_{1}^{10} x_{2}^{5} x_{3}^{3} x_{4}^{3} x_{5}^{7}, \\
& h_{2}=x_{1}^{2} x_{2}^{3} x_{3}^{5} x_{4}^{6} x_{5}^{11}+x_{1}^{2} x_{2}^{3} x_{3}^{5} x_{4}^{10} x_{5}^{7}+x_{1}^{2} x_{2}^{3} x_{3}^{9} x_{4}^{6} x_{5}^{7}+x_{1}^{2} x_{2}^{11} x_{3}^{3} x_{4}^{4} x_{5}^{7}+x_{1}^{2} x_{2}^{11} x_{3}^{4} x_{4}^{3} x_{5}^{7} \\
& +x_{1}^{3} x_{2}^{3} x_{3}^{4} x_{4}^{6} x_{5}^{11}+x_{1}^{3} x_{2}^{3} x_{3}^{4} x_{4}^{10} x_{5}^{7}+x_{1}^{3} x_{2}^{3} x_{3}^{6} x_{4}^{4} x_{5}^{11}+x_{1}^{3} x_{2}^{3} x_{3}^{6} x_{4}^{8} x_{5}^{7}+x_{1}^{3} x_{2}^{3} x_{3}^{8} x_{4}^{6} x_{5}^{7} \\
& +x_{1}^{3} x_{2}^{3} x_{3}^{10} x_{4}^{4} x_{5}^{7}+x_{1}^{3} x_{2}^{4} x_{3}^{3} x_{4}^{6} x_{5}^{11}+x_{1}^{3} x_{2}^{4} x_{3}^{3} x_{4}^{10} x_{5}^{7}+x_{1}^{3} x_{2}^{4} x_{3}^{6} x_{4}^{3} x_{5}^{11}+x_{1}^{3} x_{2}^{4} x_{3}^{10} x_{4}^{3} x_{5}^{7} \\
& +x_{1}^{3} x_{2}^{5} x_{3}^{5} x_{4}^{5} x_{5}^{9}+x_{1}^{3} x_{2}^{6} x_{3}^{2} x_{4}^{5} x_{5}^{11}+x_{1}^{3} x_{2}^{6} x_{3}^{2} x_{4}^{9} x_{5}^{7}+x_{1}^{3} x_{2}^{6} x_{3}^{3} x_{4}^{4} x_{5}^{11}+x_{1}^{3} x_{2}^{6} x_{3}^{3} x_{4}^{8} x_{5}^{7} \\
& +x_{1}^{3} x_{2}^{8} x_{3}^{3} x_{4}^{6} x_{5}^{7}+x_{1}^{3} x_{2}^{8} x_{3}^{6} x_{4}^{3} x_{5}^{7}+x_{1}^{3} x_{2}^{10} x_{3}^{2} x_{4}^{5} x_{5}^{7}+x_{1}^{3} x_{2}^{10} x_{3}^{3} x_{4}^{4} x_{5}^{7}+x_{1}^{5} x_{2}^{3} x_{3}^{6} x_{4}^{2} x_{5}^{11} \\
& +x_{1}^{5} x_{2}^{3} x_{3}^{6} x_{4}^{3} x_{5}^{10}+x_{1}^{5} x_{2}^{3} x_{3}^{6} x_{4}^{6} x_{5}^{7}+x_{1}^{5} x_{2}^{10} x_{3}^{2} x_{4}^{3} x_{5}^{7}+x_{1}^{9} x_{2}^{3} x_{3}^{3} x_{4}^{3} x_{5}^{9}+x_{1}^{9} x_{2}^{3} x_{3}^{3} x_{4}^{5} x_{5}^{7} \\
& +x_{1}^{9} x_{2}^{3} x_{3}^{5} x_{4}^{3} x_{5}^{7}+x_{1}^{9} x_{2}^{5} x_{3}^{3} x_{4}^{3} x_{5}^{7}+x_{1}^{9} x_{2}^{6} x_{3}^{2} x_{4}^{3} x_{5}^{7} \text {, } \\
& h_{4}=x_{1}^{3} x_{2}^{3} x_{3}^{6} x_{4}^{2} x_{5}^{11}+x_{1}^{3} x_{2}^{3} x_{3}^{6} x_{4}^{3} x_{5}^{10}+x_{1}^{3} x_{2}^{3} x_{3}^{6} x_{4}^{6} x_{5}^{7}+x_{1}^{3} x_{2}^{4} x_{3}^{5} x_{4}^{6} x_{5}^{7}+x_{1}^{3} x_{2}^{4} x_{3}^{6} x_{4}^{5} x_{5}^{7} \\
& +x_{1}^{3} x_{2}^{6} x_{3}^{5} x_{4}^{4} x_{5}^{7}+x_{1}^{3} x_{2}^{10} x_{3}^{2} x_{4}^{3} x_{5}^{7}+x_{1}^{5} x_{2}^{3} x_{3}^{4} x_{4}^{6} x_{5}^{7}+x_{1}^{5} x_{2}^{3} x_{3}^{6} x_{4}^{4} x_{5}^{7}+x_{1}^{5} x_{2}^{4} x_{3}^{3} x_{4}^{6} x_{5}^{7} \\
& +x_{1}^{5} x_{2}^{4} x_{3}^{6} x_{4}^{3} x_{5}^{7}+x_{1}^{5} x_{2}^{6} x_{3}^{2} x_{4}^{5} x_{5}^{7}+x_{1}^{5} x_{2}^{6} x_{3}^{3} x_{4}^{4} x_{5}^{7} \text {. }
\end{aligned}
$$

Now, let $g=x_{1} x_{2} x_{3} x_{4} x_{5}^{2}$ and $h=x_{1} x_{2} x_{3} x_{4} x_{5}$. Then, we have

$$
u:=x_{1}^{5} x_{2}^{5} x_{3}^{5} x_{4}^{5} x_{5}^{9}=h \cdot g^{4}=h S q^{12}\left(g^{2}\right) .
$$

On the other hand, by the formula $\chi$-trick (see [54]), one gets

$$
u=g^{2} \chi\left(S q^{12}\right)(h)+\sum_{1 \leqslant i \leqslant 12} S q^{i}\left(g^{2} \chi\left(S q^{12-i}\right)(h)\right) .
$$


Since $\chi\left(S q^{12}\right)=S q^{8} S q^{4}+S q^{8} S q^{3} S q^{1}$, by a direct computation, we coclude that

$$
g^{2} \chi\left(S q^{12}\right)(h) \in P_{5}^{-}\left(\omega_{(5,3)}\right) .
$$

This implies that $x$ is strictly inadmissible. The lemma follows.

Proof of Proposition 3.2.6. Let $x$ be an admissible monomial in $\left(P_{5}^{+}\right)_{29}$ such that $\omega(x)=\omega_{(5,3)}$. Then $x=X_{\{i, j\}} y^{2}$ with $y \in \mathscr{B}_{5}\left(\omega_{(5,2)}\right)$, and $1 \leqslant i<j \leqslant 5$.

Let $u \in \mathscr{B}_{5}\left(\omega_{(5,2)}\right)$ such that $X_{\{i, j\}} u^{2} \in P_{5}^{+}$. By a direct computation using Proposition 3.2.3, we see that if $X_{\{i, j\}} u^{2} \neq b_{29, k}, \forall t, 1 \leqslant k \leqslant 260$, then there is a monomial $w$ which is given in one of Lemmas 3.2.7, 3.2.8, 3.2.9 and 3.2.10 such that $X_{\{i, j\}} u^{2}=w u_{1}^{2^{\ell}}$ with suitable monomial $u_{1} \in P_{5}$, and $\ell=\max \left\{r \in \mathbb{Z}: \omega_{r}(w)>0\right\}$. By Theorem 2.2.5, $X_{\{i, j\}} u^{2}$ is inadmissible. Since $x=X_{\{i, j\}} y^{2}$ with $y \in \mathscr{B}_{5}\left(\omega_{(5,2)}\right)$, and $x$ is admissible, one can see that $x=b_{29, k}$. for some $k, 1 \leqslant k \leqslant 260$. This implies $\mathscr{B}_{5}^{+}\left(\omega_{(5,3)}\right) \subseteq\left\{b_{29, k}: 1 \leqslant k \leqslant 260\right\}$.

Now, we prove the set $\left\{\left[b_{29, k}\right]_{\omega_{(5,3)}}: 1 \leqslant k \leqslant 260\right\}$ is linearly independent in $\left(Q P_{5}\right)_{29}$. Suppose there is a linear relation

$$
\mathcal{S}=\sum_{1 \leqslant k \leqslant 260} \gamma_{k} b_{29, k} \equiv_{\omega_{(5,3)}} 0
$$

where $\gamma_{k} \in \mathbb{F}_{2}$ for all $k, 1 \leqslant k \leqslant 260$.

From Theorem 3.1.3, for $(i ; I) \in \mathcal{N}_{5}$, we explicitly compute $p_{(i ; I)}(\mathcal{S})$ in terms of a given minimal set of $\mathscr{A}$-generators in $P_{4}\left(\bmod \left(\mathscr{A}^{+} \cdot P_{4}\right)\right)$. Based on the relations $p_{(i ; I)}(\mathcal{S}) \equiv_{\omega_{(5,3)}} 0, \forall(i ; I) \in$ $\mathcal{N}_{5}, \ell(I)>0$, and some other simple computations, we get $\gamma_{k}=0, k=1,2, \ldots, 260$. The proof is completed.

Recall that $\operatorname{dim}\left(Q P_{5}^{0}\right)_{29}=195$. Combining this with Theorem 3.2.1 and Proposition 3.2.6, we obtain $\operatorname{dim}\left(Q P_{5}\right)_{29}=645$.

The case $t \geqslant 4$. We have the following.

Proposition 3.2.11. There exist exactly 270 admissible monomials in $P_{5}^{+}$such that their weight vectors are $\omega_{(5, t)}$, for every $t \geqslant 4$. Consequently $\operatorname{dim}\left(Q P_{5}^{+}\left(\omega_{(5, t)}\right)\right)=270$ for $t \geqslant 4$.

We prove the proposition by showing that

$$
\mathscr{B}_{5}^{+}\left(\omega_{(5, t)}\right)=\left\{b_{3\left(2^{t}-1\right)+2^{t}, k}: 1 \leqslant k \leqslant 270\right\},
$$

for all $t \geqslant 4$. Here, the monomials $b_{3\left(2^{t}-1\right)+2^{t}, k}, 1 \leqslant k \leqslant 270$, are listed in Subsection 5.2.

Lemma 3.2.12. If $(i, j, k, \ell, m)$ is a permutation of $(1,2,3,4,5)$ such that $i<j<k<\ell$ then the following monomials are strictly inadmissible:

$$
\begin{array}{llll}
x_{i}^{6} x_{j} x_{k} x_{\ell}^{6} x_{m}^{7}, & x_{i} x_{j}^{2} x_{k}^{6} x_{\ell}^{5} x_{m}^{7}, & x_{i} x_{j}^{6} x_{k}^{2} x_{\ell}^{5} x_{m}^{7}, & x_{i}^{6} x_{j} x_{k}^{2} x_{\ell}^{5} x_{m}^{7}, \\
x_{i} x_{j}^{6} x_{k}^{3} x_{\ell}^{4} x_{m}^{7}, & x_{i}^{3} x_{j}^{6} x_{k} x_{\ell}^{4} x_{m}^{7}, & x_{i}^{6} x_{j} x_{k}^{3} x_{\ell}^{4} x_{m}^{7}, & x_{i}^{6} x_{j}^{3} x_{k} x_{\ell}^{4} x_{m}^{7}, \\
x_{i}^{6} x_{j} x_{k}^{3} x_{\ell}^{5} x_{m}^{6}, & x_{i}^{6} x_{j}^{3} x_{k}^{5} x_{\ell} x_{m}^{6}, & x_{i}^{6} x_{j}^{3} x_{k} x_{\ell}^{5} x_{m}^{6}, & x_{i}^{2} x_{j}^{2} x_{k}^{5} x_{\ell}^{5} x_{m}^{7}, \\
x_{i}^{2} x_{j}^{5} x_{k}^{2} x_{\ell}^{5} x_{m}^{7}, & x_{i}^{2} x_{j}^{3} x_{k}^{4} x_{\ell}^{5} x_{m}^{7}, & x_{i}^{2} x_{j}^{3} x_{k}^{5} x_{\ell}^{4} x_{m}^{7}, & x_{i}^{2} x_{j}^{4} x_{k}^{3} x_{\ell}^{5} x_{m}^{7}, \\
x_{i}^{2} x_{j}^{5} x_{k}^{3} x_{\ell}^{4} x_{m}^{7}, & x_{i}^{3} x_{j}^{2} x_{k}^{4} x_{\ell}^{5} x_{m}^{7}, & x_{i}^{3} x_{j}^{2} x_{k}^{5} x_{\ell}^{4} x_{m}^{7}, & x_{i}^{3} x_{j}^{4} x_{k}^{2} x_{\ell}^{5} x_{m}^{7}, \\
x_{i}^{2} x_{j}^{5} x_{k}^{3} x_{\ell}^{5} x_{m}^{6}, & x_{i}^{3} x_{j}^{2} x_{k}^{5} x_{\ell}^{5} x_{m}^{6}, & x_{i}^{3} x_{j}^{4} x_{k}^{3} x_{\ell}^{4} x_{m}^{7}, & x_{i} x_{j}^{7} x_{k}^{10} x_{\ell}^{12} x_{m}^{15}, \\
x_{i}^{7} x_{j} x_{k}^{10} x_{\ell}^{12} x_{m}^{15}, & x_{i}^{3} x_{j}^{3} x_{k}^{12} x_{\ell}^{12} x_{m}^{15}, & x_{i}^{3} x_{j}^{5} x_{k}^{8} x_{\ell}^{14} x_{m}^{15}, & x_{i}^{3} x_{j}^{5} x_{k}^{14} x_{\ell}^{8} x_{m}^{15}, \\
x_{i}^{7} x_{j}^{7} x_{k}^{8} x_{\ell}^{8} x_{m}^{15} & &
\end{array}
$$

Proof. We prove the lemma for the monomial $x=x_{i}^{7} x_{j}^{7} x_{k}^{8} x_{\ell}^{8} x_{m}^{15}$. The others can be proved by a similar computation. 
Applying the Cartan formula, we have

$$
\begin{aligned}
x= & x_{i}^{7} x_{j}^{5} x_{k}^{10} x_{\ell}^{8} x_{m}^{15}+x_{i}^{5} x_{j}^{11} x_{k}^{6} x_{\ell}^{8} x_{m}^{15}+x_{i}^{5} x_{j}^{7} x_{k}^{10} x_{\ell}^{8} x_{m}^{15} \\
& +x_{i}^{5} x_{j}^{11} x_{k}^{10} x_{\ell}^{4} x_{m}^{15}+x_{i}^{5} x_{j}^{7} x_{k}^{10} x_{\ell}^{8} x_{m}^{15}+x_{i}^{5} x_{j}^{10} x_{k}^{11} x_{\ell}^{4} x_{m}^{15} \\
& +x_{i}^{5} x_{j}^{6} x_{k}^{11} x_{\ell}^{8} x_{m}^{15}+x_{i}^{5} x_{j}^{3} x_{k}^{14} x_{\ell}^{8} x_{m}^{15}+x_{i}^{4} x_{j}^{11} x_{k}^{11} x_{\ell}^{4} x_{m}^{15} \\
& +x_{i}^{4} x_{j}^{7} x_{k}^{11} x_{\ell}^{8} x_{m}^{15}+S q^{1}\left(x_{i}^{7} x_{j}^{7} x_{k}^{11} x_{\ell}^{4} x_{m}^{15}+x_{i}^{7} x_{j}^{5} x_{k}^{13} x_{\ell}^{4} x_{m}^{15}\right) \\
& +S q^{2}(y)+S q^{4}(z)+S q^{8}\left(x_{i}^{7} x_{j}^{5} x_{k}^{6} x_{\ell}^{4} x_{m}^{15}\right) \bmod \left(P_{5}^{-}\left(\omega_{(5,4)}^{*}\right)\right), \text { where } \\
y= & x_{i}^{7} x_{j}^{7} x_{k}^{6} x_{\ell}^{8} x_{m}^{15}+x_{i}^{7} x_{j}^{3} x_{k}^{6} x_{\ell}^{4} x_{m}^{23}+x_{i}^{7} x_{j}^{7} x_{k}^{10} x_{\ell}^{4} x_{m}^{15}+x_{i}^{7} x_{j}^{6} x_{k}^{11} x_{\ell}^{4} x_{m}^{15}+x_{i}^{7} x_{j}^{3} x_{k}^{14} x_{\ell}^{4} x_{m}^{15}, \\
z= & x_{i}^{5} x_{j}^{7} x_{k}^{6} x_{\ell}^{8} x_{m}^{15}+x_{i}^{11} x_{j}^{5} x_{k}^{6} x_{\ell}^{4} x_{m}^{15}+x_{i}^{5} x_{j}^{7} x_{k}^{10} x_{\ell}^{4} x_{m}^{15} \\
& +x_{i}^{4} x_{j}^{7} x_{k}^{11} x_{\ell}^{4} x_{m}^{15}+x_{i}^{5} x_{j}^{6} x_{k}^{11} x_{\ell}^{4} x_{m}^{15}+x_{i}^{5} x_{j}^{3} x_{k}^{14} x_{\ell}^{4} x_{m}^{15} .
\end{aligned}
$$

The above equalities imply that $x$ is strictly inadmissible.

The following lemma is proved by a direct computation.

Lemma 3.2.13. The following monomials are strictly inadmissible:

(i) $x_{i}^{6} x_{j}^{6} x_{k} x_{l} x_{m}^{7}, x_{i}^{2} x_{j} x_{k}^{4} x_{l}^{7} x_{m}^{7}, x_{i}^{2} x_{j}^{4} x_{k} x_{l}^{7} x_{m}^{7}, x_{i}^{2} x_{j}^{5} x_{k} x_{l}^{6} x_{m}^{7}, x_{i}^{2} x_{j} x_{k}^{5} x_{l}^{6} x_{m}^{7}, x_{i}^{3} x_{j}^{4} x_{k} x_{l}^{6} x_{m}^{7}$, whenever $(i, j, k, l, m)$ is a permutation of $(1,2,3,4,5)$ such that $i<j<k$.

(ii) $x_{1}^{3} x_{2}^{3} x_{3}^{6} x_{4}^{4} x_{5}^{5}, \quad x_{1}^{3} x_{2}^{3} x_{3}^{6} x_{4}^{5} x_{5}^{4}$ $x_{1}^{3} x_{2}^{6} x_{3}^{3} x_{4}^{4} x_{5}^{5}, \quad x_{1}^{3} x_{2}^{6} x_{3}^{3} x_{4}^{5} x_{5}^{4}$, $x_{1}^{6} x_{2}^{3} x_{3}^{3} x_{4}^{4} x_{5}^{5}, \quad x_{1}^{6} x_{2}^{3} x_{3}^{3} x_{4}^{5} x_{5}^{4}$ $x_{1}^{3} x_{2}^{5} x_{3}^{2} x_{4}^{6} x_{5}^{5}, \quad x_{1}^{3} x_{2}^{5} x_{3}^{6} x_{4}^{2} x_{5}^{5}$, $x_{1}^{3} x_{2}^{3} x_{3}^{4} x_{4}^{6} x_{5}^{5}, \quad x_{1}^{3} x_{2}^{5} x_{3}^{9} x_{4}^{14} x_{5}^{14}, \quad x_{1}^{3} x_{2}^{5} x_{3}^{14} x_{4}^{9} x_{5}^{14}$.

$$
\begin{array}{ll}
x_{1}^{3} x_{2}^{4} x_{3}^{3} x_{4}^{6} x_{5}^{5}, & x_{1}^{3} x_{2}^{4} x_{3}^{6} x_{4}^{3} x_{5}^{5}, \\
x_{1}^{3} x_{2}^{6} x_{3}^{4} x_{4}^{3} x_{5}^{5}, & x_{1}^{3} x_{2}^{6} x_{3}^{5} x_{4}^{3} x_{5}^{4}, \\
x_{1}^{6} x_{2}^{3} x_{3}^{4} x_{4}^{3} x_{5}^{5}, & x_{1}^{6} x_{2}^{3} x_{3}^{5} x_{4}^{3} x_{5}^{4}, \\
x_{1}^{3} x_{2}^{6} x_{3}^{5} x_{4}^{2} x_{5}^{5}, & x_{1}^{6} x_{2}^{3} x_{3}^{5} x_{4}^{2} x_{5}^{5},
\end{array}
$$

Lemma 3.2.14. The following monomials are strictly inadmissible:

(i) $\rho_{i}(f), 1 \leqslant i \leqslant 5$, where $f$ is one of the following monomials:

$$
\begin{array}{ll}
x_{1}^{2} x_{2}^{5} x_{3}^{7} x_{4}^{7}, & x_{1}^{2} x_{2}^{7} x_{3}^{5} x_{4}^{7}, \\
x_{1}^{7} x_{2}^{2} x_{3}^{7} x_{4}^{5}, & x_{1}^{7} x_{2}^{7} x_{3}^{2} x_{4}^{5}, \\
x_{1}^{3} x_{2}^{7} x_{3}^{7} x_{4}^{4}, & x_{1}^{7} x_{2}^{3} x_{3}^{4} x_{4}^{7}, \\
x_{1}^{3} x_{2}^{6} x_{3}^{5} x_{4}^{7}, & x_{1}^{3} x_{2}^{6} x_{3}^{7} x_{4}^{5}, \\
x_{1}^{6} x_{2}^{3} x_{3}^{7} x_{4}^{5}, & x_{1}^{6} x_{2}^{7} x_{3}^{3} x_{4}^{5}, \\
x_{1}^{6} x_{2} x_{3}^{7} x_{4}^{7}, & x_{1}^{6} x_{2}^{7} x_{3} x_{4}^{7}, \\
x_{1}^{7} x_{2}^{6} x_{3}^{7} x_{4}, & x_{1}^{7} x_{2}^{7} x_{3}^{6} x_{4} .
\end{array}
$$

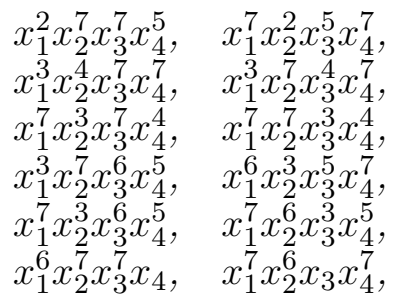

(ii)

$\begin{array}{lllll}x_{1} x_{2}^{15} x_{3}^{15} x_{4}^{18} x_{5}^{12}, & x_{1}^{15} x_{2} x_{3}^{15} x_{4}^{18} x_{5}^{12}, & x_{1}^{15} x_{2}^{15} x_{3} x_{4}^{18} x_{5}^{12}, & x_{1} x_{2}^{14} x_{3}^{15} x_{4}^{15} x_{5}^{16}, \\ x_{1} x_{2}^{15} x_{3}^{14} x_{4}^{15} x_{5}^{16}, & x_{1}^{15} x_{2} x_{3}^{14} x_{4}^{15} x_{5}^{16}, & x_{1} x_{2}^{15} x_{3}^{15} x_{4}^{14} x_{5}^{16}, & x_{1}^{15} x_{2} x_{3}^{15} x_{4}^{14} x_{5}^{16}, \\ x_{1}^{15} x_{2}^{15} x_{3} x_{4}^{14} x_{5}^{16}, & x_{1}^{15} x_{2}^{15} x_{3}^{17} x_{4}^{2} x_{5}^{12}, & x_{1}^{3} x_{2}^{13} x_{3}^{14} x_{4}^{19} x_{5}^{12}, & x_{1}^{3} x_{2}^{13} x_{3}^{15} x_{4}^{18} x_{5}^{12}, \\ x_{1}^{3} x_{2}^{15} x_{3}^{13} x_{4}^{18} x_{5}^{12}, & x_{1}^{15} x_{2}^{3} x_{3}^{13} x_{4}^{18} x_{5}^{12}, & x_{1}^{3} x_{2}^{13} x_{3}^{14} x_{4}^{15} x_{5}^{16}, & x_{1}^{3} x_{2}^{13} x_{3}^{15} x_{4}^{14} x_{5}^{16}, \\ x_{1}^{3} x_{2}^{15} x_{3}^{13} x_{4}^{14} x_{5}^{16}, & x_{1}^{15} x_{2}^{3} x_{3}^{13} x_{4}^{14} x_{5}^{16}, & x_{1}^{7} x_{2}^{7} x_{3}^{8} x_{4}^{9} x_{5}^{30}, & x_{1}^{7} x_{2}^{7} x_{3}^{9} x_{4}^{8} x_{5}^{30}, \\ x_{1}^{7} x_{2}^{7} x_{3}^{9} x_{4}^{30} x_{5}^{8}, & x_{1}^{7} x_{2}^{7} x_{3}^{9} x_{4}^{14} x_{5}^{24}, & x_{1}^{7} x_{2}^{11} x_{3}^{13} x_{4}^{16} x_{5}^{14}, & x_{1}^{7} x_{2}^{15} x_{3}^{17} x_{4}^{10} x_{5}^{12}, \\ x_{1}^{15} x_{2}^{7} x_{3}^{17} x_{4}^{10} x_{5}^{12}, & x_{1}^{15} x_{2}^{19} x_{3}^{5} x_{4}^{10} x_{5}^{12} . & & \end{array}$

Proof. The first part of the lemma is an immediate corollary from a result in [47]. We prove the second part of this lemma for the monomials $x=x_{1} x_{2}^{14} x_{3}^{15} x_{4}^{15} x_{5}^{16}$ and $y=x_{1}^{7} x_{2}^{7} x_{3}^{9} x_{4}^{8} x_{5}^{30}$. The others 
can be proved by a similar computation. By a direct computation, one gets

$$
\begin{aligned}
x= & x_{1} x_{2}^{10} x_{3}^{12} x_{4}^{15} x_{5}^{23}+x_{1} x_{2}^{10} x_{3}^{12} x_{4}^{23} x_{5}^{15}+x_{1} x_{2}^{10} x_{3}^{14} x_{4}^{13} x_{5}^{23}+x_{1} x_{2}^{10} x_{3}^{14} x_{4}^{21} x_{5}^{15} \\
& +x_{1} x_{2}^{10} x_{3}^{20} x_{4}^{15} x_{5}^{15}+x_{1} x_{2}^{10} x_{3}^{22} x_{4}^{13} x_{5}^{15}+x_{1} x_{2}^{12} x_{3}^{15} x_{4}^{10} x_{5}^{23}+x_{1} x_{2}^{12} x_{3}^{15} x_{4}^{15} x_{5}^{18} \\
& +x_{1} x_{2}^{12} x_{3}^{18} x_{4}^{15} x_{5}^{15}+x_{1} x_{2}^{12} x_{3}^{26} x_{4}^{7} x_{5}^{15}+x_{1} x_{2}^{14} x_{3}^{12} x_{4}^{15} x_{5}^{19}+x_{1} x_{2}^{14} x_{3}^{12} x_{4}^{19} x_{5}^{15} \\
& +x_{1} x_{2}^{14} x_{3}^{14} x_{4}^{13} x_{5}^{19}+x_{1} x_{2}^{14} x_{3}^{14} x_{4}^{17} x_{5}^{15}+x_{1} x_{2}^{14} x_{3}^{15} x_{4}^{8} x_{5}^{23} \\
& +S q^{1}\left(f_{1}\right)+S q^{2}\left(f_{2}\right)+S q^{4}\left(f_{4}\right)+S q^{8}\left(f_{8}\right) \bmod \left(P_{5}^{-}\left(\omega_{(5,4)}\right)\right), \text { where } \\
f_{1}= & x_{1}^{2} x_{2}^{11} x_{3}^{15} x_{4}^{15} x_{5}^{17}+x_{1}^{2} x_{2}^{11} x_{3}^{15} x_{4}^{17} x_{5}^{15}+x_{1}^{2} x_{2}^{11} x_{3}^{17} x_{4}^{15} x_{5}^{15}+x_{1}^{2} x_{2}^{11} x_{3}^{23} x_{4}^{7} x_{5}^{17} \\
& +x_{1}^{2} x_{2}^{11} x_{3}^{23} x_{4}^{9} x_{5}^{15}+x_{1}^{2} x_{2}^{11} x_{3}^{25} x_{4}^{7} x_{5}^{15}+x_{1}^{2} x_{2}^{13} x_{3}^{15} x_{4}^{15} x_{5}^{15}+x_{1}^{2} x_{2}^{13} x_{3}^{23} x_{4}^{7} x_{5}^{15}, \\
f_{2}= & x_{1} x_{2}^{11} x_{3}^{15} x_{4}^{15} x_{5}^{17}+x_{1} x_{2}^{11} x_{3}^{15} x_{4}^{17} x_{5}^{15}+x_{1} x_{2}^{11} x_{3}^{17} x_{4}^{15} x_{5}^{15}+x_{1} x_{2}^{11} x_{3}^{23} x_{4}^{7} x_{5}^{17} \\
& +x_{1} x_{2}^{11} x_{3}^{23} x_{4}^{9} x_{5}^{15}+x_{1} x_{2}^{11} x_{3}^{25} x_{4}^{7} x_{5}^{15}+x_{1} x_{2}^{13} x_{3}^{15} x_{4}^{15} x_{5}^{15}+x_{1} x_{2}^{13} x_{3}^{23} x_{4}^{7} x_{5}^{15} \\
& +x_{1} x_{2}^{14} x_{3}^{18} x_{4}^{11} x_{5}^{15}+x_{1} x_{2}^{22} x_{3}^{15} x_{4}^{6} x_{5}^{15}, \\
f_{4}= & x_{1} x_{2}^{14} x_{3}^{12} x_{4}^{15} x_{5}^{15}+x_{1} x_{2}^{14} x_{3}^{14} x_{4}^{13} x_{5}^{15}+x_{1} x_{2}^{14} x_{3}^{20} x_{4}^{7} x_{5}^{15}+x_{1} x_{2}^{20} x_{3}^{15} x_{4}^{6} x_{5}^{15}, \\
f_{8}= & x_{1} x_{2}^{10} x_{3}^{12} x_{4}^{15} x_{5}^{15}+x_{1} x_{2}^{10} x_{3}^{14} x_{4}^{13} x_{5}^{15}+x_{1} x_{2}^{12} x_{3}^{15} x_{4}^{10} x_{5}^{15}+x_{1} x_{2}^{14} x_{3}^{15} x_{4}^{8} x_{5}^{15} .
\end{aligned}
$$

The above relations show that $x$ is strictly inadmissible. By a similar computation, we obtain

$$
\begin{aligned}
& y=x_{1}^{4} x_{2}^{11} x_{3}^{12} x_{4}^{19} x_{5}^{15}+x_{1}^{4} x_{2}^{11} x_{3}^{19} x_{4}^{12} x_{5}^{15}+x_{1}^{4} x_{2}^{20} x_{3}^{11} x_{4}^{11} x_{5}^{15}+x_{1}^{4} x_{2}^{24} x_{3}^{7} x_{4}^{11} x_{5}^{15} \\
& +x_{1}^{5} x_{2}^{3} x_{3}^{12} x_{4}^{26} x_{5}^{15}+x_{1}^{5} x_{2}^{3} x_{3}^{26} x_{4}^{12} x_{5}^{15}+x_{1}^{5} x_{2}^{7} x_{3}^{8} x_{4}^{14} x_{5}^{27}+x_{1}^{5} x_{2}^{7} x_{3}^{8} x_{4}^{26} x_{5}^{15} \\
& +x_{1}^{5} x_{2}^{7} x_{3}^{10} x_{4}^{12} x_{5}^{27}+x_{1}^{5} x_{2}^{7} x_{3}^{10} x_{4}^{24} x_{5}^{15}+x_{1}^{5} x_{2}^{10} x_{3}^{12} x_{4}^{19} x_{5}^{15}+x_{1}^{5} x_{2}^{10} x_{3}^{19} x_{4}^{12} x_{5}^{15} \\
& +x_{1}^{5} x_{2}^{11} x_{3}^{5} x_{4}^{10} x_{5}^{30}+x_{1}^{5} x_{2}^{11} x_{3}^{6} x_{4}^{12} x_{5}^{27}+x_{1}^{5} x_{2}^{11} x_{3}^{8} x_{4}^{14} x_{5}^{23}+x_{1}^{5} x_{2}^{11} x_{3}^{8} x_{4}^{22} x_{5}^{15} \\
& +x_{1}^{5} x_{2}^{11} x_{3}^{9} x_{4}^{6} x_{5}^{30}+x_{1}^{5} x_{2}^{11} x_{3}^{10} x_{4}^{20} x_{5}^{15}+x_{1}^{5} x_{2}^{11} x_{3}^{16} x_{4}^{14} x_{5}^{15}+x_{1}^{5} x_{2}^{11} x_{3}^{18} x_{4}^{12} x_{5}^{15} \\
& +x_{1}^{5} x_{2}^{18} x_{3}^{11} x_{4}^{12} x_{5}^{15}+x_{1}^{7} x_{2}^{3} x_{3}^{12} x_{4}^{24} x_{5}^{15}+x_{1}^{7} x_{2}^{3} x_{3}^{24} x_{4}^{12} x_{5}^{15}+x_{1}^{7} x_{2}^{5} x_{3}^{9} x_{4}^{10} x_{5}^{30} \\
& +x_{1}^{7} x_{2}^{5} x_{3}^{10} x_{4}^{12} x_{5}^{27}+x_{1}^{7} x_{2}^{7} x_{3}^{8} x_{4}^{12} x_{5}^{27}+x_{1}^{7} x_{2}^{7} x_{3}^{8} x_{4}^{14} x_{5}^{25}+x_{1}^{7} x_{2}^{7} x_{3}^{8} x_{4}^{24} x_{5}^{15} \\
& +S q^{1}\left(g_{1}\right)+S q^{2}\left(g_{2}\right)+S q^{4}\left(g_{4}\right)+S q^{8}\left(g_{8}\right) \bmod \left(P_{5}^{-}\left(\omega_{(5,4)}\right)\right) \text {, where } \\
& g_{1}=x_{1}^{7} x_{2}^{5} x_{3}^{12} x_{4}^{21} x_{5}^{15}+x_{1}^{7} x_{2}^{5} x_{3}^{21} x_{4}^{12} x_{5}^{15}+x_{1}^{7} x_{2}^{7} x_{3}^{5} x_{4}^{12} x_{5}^{29}+x_{1}^{7} x_{2}^{7} x_{3}^{12} x_{4}^{19} x_{5}^{15} \\
& +x_{1}^{7} x_{2}^{7} x_{3}^{19} x_{4}^{12} x_{5}^{15}+x_{1}^{7} x_{2}^{9} x_{3}^{8} x_{4}^{13} x_{5}^{23}+x_{1}^{7} x_{2}^{9} x_{3}^{8} x_{4}^{21} x_{5}^{15}+x_{1}^{7} x_{2}^{9} x_{3}^{16} x_{4}^{13} x_{5}^{15} \\
& +x_{1}^{7} x_{2}^{17} x_{3}^{9} x_{4}^{12} x_{5}^{15}+x_{1}^{7} x_{2}^{20} x_{3}^{7} x_{4}^{11} x_{5}^{15} \text {, } \\
& g_{2}=x_{1}^{7} x_{2}^{3} x_{3}^{5} x_{4}^{6} x_{5}^{38}+x_{1}^{7} x_{2}^{3} x_{3}^{6} x_{4}^{20} x_{5}^{23}+x_{1}^{7} x_{2}^{3} x_{3}^{12} x_{4}^{22} x_{5}^{15}+x_{1}^{7} x_{2}^{3} x_{3}^{22} x_{4}^{12} x_{5}^{15} \\
& +x_{1}^{7} x_{2}^{6} x_{3}^{12} x_{4}^{19} x_{5}^{15}+x_{1}^{7} x_{2}^{6} x_{3}^{19} x_{4}^{12} x_{5}^{15}+x_{1}^{7} x_{2}^{7} x_{3}^{5} x_{4}^{10} x_{5}^{30}+x_{1}^{7} x_{2}^{7} x_{3}^{6} x_{4}^{12} x_{5}^{27} \\
& +x_{1}^{7} x_{2}^{7} x_{3}^{8} x_{4}^{14} x_{5}^{23}+x_{1}^{7} x_{2}^{7} x_{3}^{8} x_{4}^{22} x_{5}^{15}+x_{1}^{7} x_{2}^{7} x_{3}^{9} x_{4}^{6} x_{5}^{30}+x_{1}^{7} x_{2}^{7} x_{3}^{10} x_{4}^{20} x_{5}^{15} \\
& +x_{1}^{7} x_{2}^{7} x_{3}^{16} x_{4}^{14} x_{5}^{15}+x_{1}^{7} x_{2}^{7} x_{3}^{18} x_{4}^{12} x_{5}^{15}+x_{1}^{7} x_{2}^{18} x_{3}^{7} x_{4}^{12} x_{5}^{15}+x_{1}^{7} x_{2}^{18} x_{3}^{8} x_{4}^{11} x_{5}^{15} \text {, } \\
& g_{4}=x_{1}^{4} x_{2}^{7} x_{3}^{12} x_{4}^{19} x_{5}^{15}+x_{1}^{4} x_{2}^{7} x_{3}^{19} x_{4}^{12} x_{5}^{15}+x_{1}^{4} x_{2}^{20} x_{3}^{7} x_{4}^{11} x_{5}^{15}+x_{1}^{5} x_{2}^{3} x_{3}^{12} x_{4}^{22} x_{5}^{15} \\
& +x_{1}^{5} x_{2}^{3} x_{3}^{22} x_{4}^{12} x_{5}^{15}+x_{1}^{5} x_{2}^{6} x_{3}^{12} x_{4}^{19} x_{5}^{15}+x_{1}^{5} x_{2}^{6} x_{3}^{19} x_{4}^{12} x_{5}^{15}+x_{1}^{5} x_{2}^{7} x_{3}^{5} x_{4}^{10} x_{5}^{30} \\
& +x_{1}^{5} x_{2}^{7} x_{3}^{6} x_{4}^{12} x_{5}^{27}+x_{1}^{5} x_{2}^{7} x_{3}^{8} x_{4}^{14} x_{5}^{23}+x_{1}^{5} x_{2}^{7} x_{3}^{8} x_{4}^{22} x_{5}^{15}+x_{1}^{5} x_{2}^{7} x_{3}^{9} x_{4}^{6} x_{5}^{30} \\
& +x_{1}^{5} x_{2}^{7} x_{3}^{10} x_{4}^{20} x_{5}^{15}+x_{1}^{5} x_{2}^{7} x_{3}^{16} x_{4}^{14} x_{5}^{15}+x_{1}^{5} x_{2}^{7} x_{3}^{18} x_{4}^{12} x_{5}^{15}+x_{1}^{5} x_{2}^{18} x_{3}^{7} x_{4}^{12} x_{5}^{15} \\
& +x_{1}^{11} x_{2}^{5} x_{3}^{5} x_{4}^{6} x_{5}^{30}+x_{1}^{11} x_{2}^{5} x_{3}^{6} x_{4}^{12} x_{5}^{23} \text {, } \\
& g_{8}=x_{1}^{7} x_{2}^{5} x_{3}^{5} x_{4}^{6} x_{5}^{30}+x_{1}^{7} x_{2}^{5} x_{3}^{6} x_{4}^{12} x_{5}^{23}+x_{1}^{7} x_{2}^{9} x_{3}^{10} x_{4}^{12} x_{5}^{15}+x_{1}^{7} x_{2}^{10} x_{3}^{8} x_{4}^{13} x_{5}^{15} \text {. }
\end{aligned}
$$

By Definition 2.2.4, we see that $y$ is strictly inadmissible. The lemma follows. 
Lemma 3.2.15. For any $t \geqslant 4$, the following monomials are strictly inadmissible:

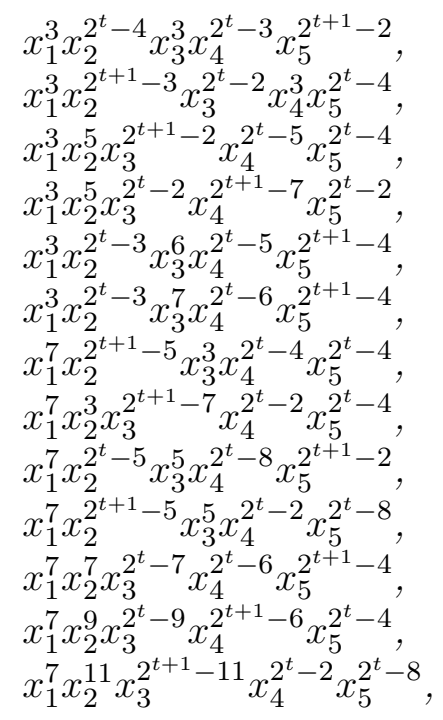

$$
\begin{aligned}
& x_{1}^{3} x_{2}^{2^{t}-3} x_{3}^{2^{t+1}-2} x_{4}^{3} x_{5}^{2^{t}-4}, \\
& x_{1}^{3} x_{2}^{2^{t+1}-4} x_{3}^{3} x_{4}^{2^{t}-3} x_{5}^{2^{t}-2}, \\
& x_{1}^{3} x_{2}^{5} x_{3}^{2^{t}-2} x_{4}^{2^{2}-5} x_{5}^{2^{t+1}-4}, \\
& x_{1}^{3} x_{2}^{5} x_{3}^{2^{t+1}-7} x_{4}^{2^{t}-2} x_{5}^{2^{t}-2}, \\
& x_{1}^{3} x_{2}^{2^{-}-3} x_{3}^{6} x_{4}^{2^{t+1}-5} x_{5}^{2^{t}-4}, \\
& x_{1}^{3} x_{2}^{7} x_{3}^{2^{t+1}-5} x_{4}^{2^{t}-4} x_{5}^{2^{t}-4}, \\
& x_{1}^{3} x_{2}^{2^{t}-3} x_{3}^{7} x_{4}^{2^{t+1}-6} x_{5}^{2^{t}-4}, \\
& x_{1}^{3} x_{2}^{13} x_{3}^{2^{t+1}-9} x_{4}^{2^{t}-6} x_{5}^{2^{t}-4}, \\
& x_{1}^{7} x_{2}^{2^{t}-5} x_{3}^{5} x_{4}^{2^{t+1}-2} x_{5}^{2^{t}-8}, \\
& x_{1}^{7} x_{2}^{2^{t}-5} x_{3}^{5} x_{4}^{2^{t}-2} x_{5}^{2^{t+1}-8}, \\
& x_{1}^{7} x_{2}^{9} x_{3}^{2^{t}-9} x_{4}^{2^{2}-6} x_{5}^{2^{t+1}-4}, \\
& x_{1}^{7} x_{2}^{7} x_{3}^{2^{t+1}-7} x_{4}^{2^{t}-6} x_{5}^{2^{t}-4}, \\
& x_{1}^{7} x_{2}^{9} x_{3}^{2^{t+1}-9} x_{4}^{2^{t}-6} x_{5}^{2^{t}-4}
\end{aligned}
$$

Proof. We prove the lemma for $u=x_{1}^{7} x_{2}^{9} x_{3}^{2^{t+1}-9} x_{4}^{2^{t}-6} x_{5}^{2^{t}-4}$. The others can be proven by a similar computation. By a direct computation using the Cartan formula, we have

$$
\begin{aligned}
u= & x_{1}^{4} x_{2}^{7} x_{3}^{2^{t+1}-5} x_{4}^{2^{t}-5} x_{5}^{2^{t}-4}+x_{1}^{4} x_{2}^{11} x_{3}^{2^{t+1}-9} x_{4}^{2^{t}-5} x_{5}^{2^{t}-4}+x_{1}^{5} x_{2}^{7} x_{3}^{2^{t+1}-5} x_{4}^{2^{t}-6} x_{5}^{2^{t}-4} \\
& +x_{1}^{5} x_{2}^{11} x_{3}^{2^{t+1}-9} x_{4}^{2^{t}-6} x_{5}^{2^{t}-4}+x_{1}^{7} x_{2}^{7} x_{3}^{2^{t+1}-8} x_{4}^{2^{t}-5} x_{5}^{2^{t}-4}+x_{1}^{7} x_{2}^{7} x_{3}^{2^{t+1}-7} x_{4}^{2^{t}-6} x_{5}^{2^{t}-4} \\
& +x_{1}^{7} x_{2}^{8} x_{3}^{2^{t+1}-9} x_{4}^{2^{t}-5} x_{5}^{2^{t}-4}+S q^{1}\left(x_{1}^{7} x_{2}^{7} x_{3}^{2^{t+1}-9} x_{4}^{2^{t}-5} x_{5}^{2^{t}-4}\right)+S q^{2}\left(x_{1}^{7} x_{2}^{7} x_{3}^{2^{t+1}-9} x_{4}^{2^{t}-6} x_{5}^{2^{t}-4}\right) \\
& +S q^{4}\left(x_{1}^{4} x_{2}^{7} x_{3}^{2^{t+1}-9} x_{4}^{2^{t}-5} x_{5}^{2^{t}-4}+x_{1}^{5} x_{2}^{7} x_{3}^{2^{t+1}-9} x_{4}^{2^{t}-6} x_{5}^{2^{t}-4}\right) \bmod \left(P_{5}^{-}\left(\omega_{(5, t)}\right)\right) .
\end{aligned}
$$

This equality shows that $u$ is strictly inadmissible. The lemma is proved.

The proof of the following lemma is analogous to the proof of Lemma 3.2.15.

Lemma 3.2.16. For any $t \geqslant 5$, the following monomials are strictly inadmissible:

$$
\begin{array}{llll}
x_{1}^{3} x_{2}^{13} x_{3}^{2^{t}-10} x_{4}^{2^{t}-5} x_{5}^{2^{t+1}-4}, & x_{1}^{3} x_{2}^{13} x_{3}^{2^{t}-10} x_{4}^{2^{t+1}-5} x_{5}^{2^{t}-4}, & x_{1}^{3} x_{2}^{13} x_{3}^{2^{t}-9} x_{4}^{2^{t}-6} x_{5}^{2^{t+1}-4}, \\
x_{1}^{3} x_{2}^{13} x_{3}^{2^{t}-9} x_{4}^{2^{t+1}-6} x_{5}^{2^{t}-4}, & x_{1}^{3} x_{2}^{15} x_{3}^{2^{t}-11} x_{4}^{2^{t}-6} x_{5}^{2^{t+1}-4}, & x_{1}^{15} x_{2}^{3} x_{3}^{2^{t}-11} x_{4}^{2^{t}-6} x_{5}^{2^{t+1}-4}, \\
x_{1}^{3} x_{2}^{15} x_{3}^{2^{t}-11} x_{4}^{2^{t+1}-6} x_{5}^{2^{t}-4}, & x_{1}^{15} x_{2}^{3} x_{3}^{2^{t}-11} x_{4}^{2^{t+1}-6} x_{5}^{2^{t}-4}, & x_{1}^{7} x_{2}^{11} x_{3}^{2^{t}-11} x_{4}^{2^{t}-8} x_{5}^{2^{t+1}-2}, \\
x_{1}^{7} x_{2}^{11} x_{3}^{2^{t}-11} x_{4}^{2^{t+1}-2} x_{5}^{2^{t}-8}, & x_{1}^{7} x_{2}^{11} x_{3}^{2^{t}-11} x_{4}^{2^{t}-2} x_{5}^{2^{t+1}-8}, & x_{1}^{7} x_{2}^{11} x_{3}^{2^{t}-11} x_{4}^{2^{t+1}-8} x_{5}^{2^{t}-2}
\end{array}
$$

Proof of Proposition 3.2.11. Let $x$ be an admissible monomial in $\left(P_{5}^{+}\right)_{3\left(2^{t}-1\right)+2^{t}}$ such that $\omega(x)=$ $\omega_{(5, t)}$ with $t \geqslant 4$. By induction on $t$, we see that $x \neq b_{3\left(2^{t}-1\right)+2^{t}, k}$ for $k=1,2, \ldots, 270$, then there is a monomial $z$, which is given in one of Lemmas 3.2.12, 3.2.13, 3.2.14, 3.2.15, and 3.2.16 such that $x=z y^{2^{\alpha}}$ for some monomial $y$ and positive integer $\alpha$. By Theorem 2.2.5, $x$ is inadmissible. Hence $\mathscr{B}_{5}^{+}\left(\omega_{(5, t)}\right) \subseteq\left\{b_{3\left(2^{t}-1\right)+2^{t}, k}: 1 \leqslant k \leqslant 270\right\}$ for every $t \geqslant 4$.

Now we prove that the classes $\left[b_{3\left(2^{t}-1\right)+2^{t}, k}\right]_{\omega_{(5, t)}} \neq[0]_{\omega_{(5, t)}}$ with $1 \leqslant k \leqslant 270$, and $t \geqslant 4$. Suppose there is a linear relation

$$
\mathcal{S}=\sum_{1 \leqslant k \leqslant 270} \gamma_{k} b_{3\left(2^{t}-1\right)+2^{t}, k} \equiv_{\omega_{(5, t)}} 0,
$$

where $\gamma_{k} \in \mathbb{F}_{2}$ for all $k, 1 \leqslant k \leqslant 270$.

For $t \geqslant 4$, according to Sum [47], $\mathscr{B}_{4}^{+}\left(3\left(2^{t}-1\right)+2^{t}\right)$ is the set consisting of 33 monomials, namely: 

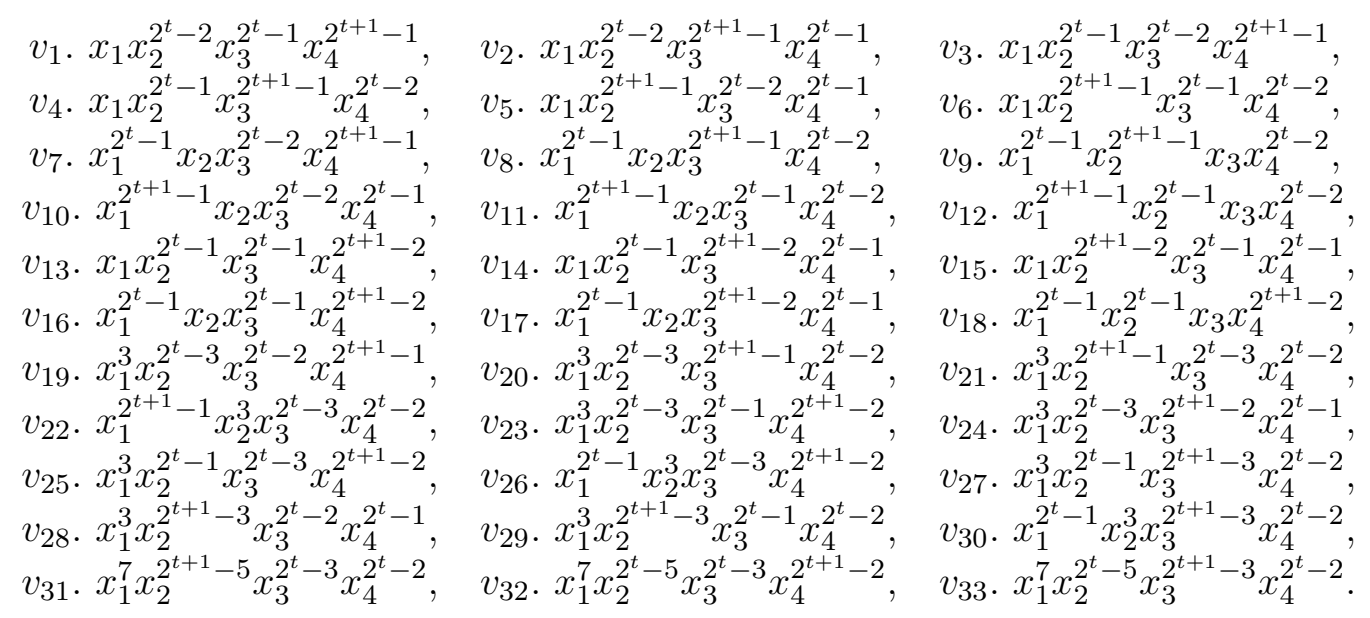

By a direct computation using Theorem 3.1.3, we express $p_{(i ; I)}(\mathcal{S})$, in terms of $v_{j}, 1 \leqslant j \leqslant 33$. Computing directly from the relations

$$
p_{(i ; I)}(\mathcal{S}) \equiv_{\omega_{(5, t)}} 0, \forall(i ; I) \in \mathcal{N}_{5}, \ell(I)=1,
$$

we obtain $\gamma_{k}=0, k=1,2, \ldots, 270$. The proposition follows.

We knew that

$$
\begin{array}{ll}
\left(Q P_{5}\right)_{3\left(2^{t}-1\right)+2^{t}} & =\operatorname{Ker}\left(\left(\widetilde{S q_{*}^{0}}\right)_{\left(5,3\left(2^{t}-1\right)+2^{t}\right)}\right)\left(\left(Q P_{5}\right)_{2^{t+1}-4},\right. \\
\operatorname{Ker}\left(\left(\widetilde{S q_{*}^{0}}\right)_{\left(5,3\left(2^{t}-1\right)+2^{t}\right)}\right) & =\left(Q P_{5}^{0}\right)_{3\left(2^{t}-1\right)+2^{t}} \oplus Q P_{5}^{+}\left(\omega_{(5, t)}\right)
\end{array}
$$

and $\operatorname{dim}\left(Q P_{5}^{0}\right)_{3\left(2^{t}-1\right)+2^{t}}=195$ for any $t \geqslant 3$. So, from Theorem 3.2.1 and Proposition 3.2.11, we get

$$
\operatorname{dim}\left(Q P_{5}\right)_{3\left(2^{t}-1\right)+2^{t}}= \begin{cases}945 & \text { if } t=4 \\ 1115 & \text { if } t=5 \\ 1116 & \text { if } t \geqslant 6\end{cases}
$$

The proof of Theorem 1.1 is completed.

\section{Proof of Theorem 1.3}

In this section, we prove Theorem 1.3 by using the results in Section 3. Firstly, we need some notations and definitions for the proof of the theorem.

For $1 \leqslant i \leqslant s$, define the $\mathscr{A}$-homomorphism $\tau_{i}: P_{s} \longrightarrow P_{s}$, which is determined by $\tau_{i}\left(x_{i}\right)=$ $x_{i+1}, \tau_{i}\left(x_{i+1}\right)=x_{i}, \tau_{i}\left(x_{j}\right)=x_{j}$ for $j \neq i, i+1,1 \leqslant i<s$ and $\tau_{s}\left(x_{1}\right)=x_{1}+x_{2}, \tau_{s}\left(x_{j}\right)=x_{j}$ for $j>1$. Observe that the general linear group $G L_{s}$ is generated by the matrices associated with by $\tau_{i}, 0 \leqslant i \leqslant s$ and the symmetric group $\Sigma_{s} \subset G L_{s}$ is generated by the ones associated with $\tau_{i}, 1 \leqslant i<s$. Hence, a class $[f]_{\omega}$ represented by a homogeneous polynomial $f \in P_{s}$ is an $G L_{s^{-}}$ invariant if and only if $\tau_{i}(f) \equiv_{\omega} f$ for $1 \leqslant i \leqslant s$. $[f]_{\omega}$ is an $\Sigma_{s}$-invariant if and only if $\tau_{i}(f) \equiv_{\omega} f$ for $1 \leqslant i<s$.

For the weight vector $\omega_{(5, t)}=(3,3, \ldots, 3,1)(t$ times of 3$)$, we have $\operatorname{deg} \omega_{(5, t)}=3\left(2^{t}-1\right)+2^{t}$. For any monomials $g_{1}, g_{2}, \ldots, g_{m}$ in $P_{5}\left(\omega_{(5, t)}\right)$ and for a subgroup $G \subset G L_{5}$, we denote $G\left(g_{1}, \ldots, g_{m}\right)$ the $G$-submodule of $Q P_{5}\left(\omega_{(5, t)}\right)$ generated by the set $\left\{\left[g_{i}\right]_{\omega_{(5, t)}}: 1 \leqslant i \leqslant m\right\}$. We see that $\omega_{(5, t)}$ is the weight vector of the mimimal spike $x_{1}^{2^{t+1}-1} x_{2}^{2^{t}-1} x_{3}^{2^{t}-1} \in\left(P_{5}\right)_{3\left(2^{t}-1\right)+2^{t}}$; hence $\left[g_{i}\right]_{\omega_{(5, t)}}=\left[g_{i}\right]$ for all $i, 1 \leqslant i \leqslant m$.

Recall that Kameko's map $\widetilde{S q_{*}^{0}}=\left(\widetilde{S q_{*}^{0}}\right)_{\left(5,3\left(2^{t}-1\right)+2^{t}\right)}:\left(Q P_{5}\right)_{3\left(2^{t}-1\right)+2^{t}} \longrightarrow\left(Q P_{5}\right)_{2^{t+1}-4}$ is an epimorphism of $G L_{5}$-modules. Then, as mentioned in Section 1, we need only to determine $\operatorname{Ker}\left(\left(\widetilde{S q_{*}^{0}}\right)_{\left(5,3\left(2^{t}-1\right)+2^{t}\right)}\right)^{G L_{5}}$. 
From the results in Section 3, we get

$$
\operatorname{dim}\left(\operatorname{Ker}\left(\left(\widetilde{S q_{*}^{0}}\right)_{\left(5,3\left(2^{t}-1\right)+2^{t}\right)}\right)\right)=\operatorname{dim}\left(Q P_{5}\left(\omega_{(5, t)}\right)\right) .
$$

Note that $Q P_{5}\left(\omega_{(5, t)}\right)=Q P_{5}^{0}\left(\omega_{(5, t)}\right) \oplus Q P_{5}^{+}\left(\omega_{(5, t)}\right)$ and

$$
\operatorname{dim}\left(\operatorname{Ker}\left(\left(\widetilde{S q_{*}^{0}}\right)_{\left(5,3\left(2^{t}-1\right)+2^{t}\right)}\right)^{G L_{5}}\right) \leqslant \operatorname{dim}\left(Q P_{5}\left(\omega_{(5, t)}\right)^{G L_{5}}\right) .
$$

Now, for $t=1,3\left(2^{t}-1\right)+2^{t}=5$. From a result of Sum [46, 50], we have $Q P_{5}\left(\omega_{(5,1)}\right)^{G L_{5}}=0$.

We now compute $Q P_{5}\left(\omega_{(5, t)}\right)^{G L_{5}}$ for $t \geqslant 2$.

\subsection{Computation of $Q P_{5}\left(\omega_{(5, t)}\right)^{\Sigma_{5}}$}

As it is known, $\mathscr{B}_{5}^{0}\left(3\left(2^{t}-1\right)+2^{t}\right)=\mathscr{B}_{5}^{0}\left(\omega_{(5, t)}\right)$ is the set consisting of the admissible monomials $q_{t, k}$ (see Subsection 5.1). By a simple computation, we see that the following subspaces are $\Sigma_{5^{-}}$ submodules of $Q P_{5}^{0}\left(\omega_{(5, t)}\right)$ :

For $t \geqslant 2$,

$$
\begin{aligned}
& \Sigma_{5}\left(q_{t, 1}\right)=\left\langle\left[q_{t, k}\right]_{\omega_{(5, t)}}: 1 \leqslant k \leqslant 30\right\rangle, \\
& \Sigma_{5}\left(q_{t, 31}\right)=\left\langle\left[q_{t, k}\right]_{\omega_{(5, t)}}: 31 \leqslant k \leqslant 90\right\rangle .
\end{aligned}
$$

For $t=2$,

$$
\mathcal{M}_{1}=\left\langle\left[q_{2, k}\right]_{\omega_{(5,2)}}: 91 \leqslant k \leqslant 145\right\rangle \text {. }
$$

For $t \geqslant 3$,

$$
\Sigma_{5}\left(q_{t, 91}\right)=\left\langle\left[q_{t, k}\right]_{\omega_{(5, t)}}: 91 \leqslant k \leqslant 110\right\rangle
$$

For $t=3$,

$$
\mathcal{M}_{2}=\left\langle\left[q_{3, k}\right]_{\omega_{(5,3)}}: 111 \leqslant k \leqslant 195\right\rangle
$$

For $t \geqslant 4$,

$$
\mathcal{M}_{t, 1}^{*}=\left\langle\left[q_{t, k}\right]_{\omega_{(5, t)}}: 111 \leqslant k \leqslant 195\right\rangle .
$$

Lemma 4.1.1. Let $t$ be an integer. Then, we obtain the following:

For $t \geqslant 2$,

$$
\begin{aligned}
& \Sigma_{5}\left(q_{t, 1}\right)^{\Sigma_{5}}=\left\langle\left[p_{t, 1}:=q_{t, 1}+q_{t, 2}+\cdots+q_{t, 30}\right]_{\omega_{(5, t)}}\right\rangle, \\
& \Sigma_{5}\left(q_{t, 31}\right)^{\Sigma_{5}}=\left\langle\left[p_{t, 2}:=q_{t, 31}+q_{t, 32}+\cdots+q_{t, 90}\right]_{\omega_{(5, t)}}\right\rangle .
\end{aligned}
$$

For $t \geqslant 3$,

$$
\Sigma_{5}\left(q_{t, 91}\right)^{\Sigma_{5}}=\left\langle\left[p_{t, 3}:=q_{t, 91}+q_{t, 92}+\cdots+q_{t, 110}\right]_{\omega_{(5, t)}}\right\rangle .
$$

Outline of the proof. The set $B:=\left\{\left[q_{t, k}\right]_{\omega_{(5, t)}}: 1 \leqslant k \leqslant 30\right\}$ is a basis of $\Sigma_{5}\left(q_{t, 1}\right)$ for $t \geqslant 2$. The action of $\Sigma_{5}$ on $Q P_{5}$ induces the one of it on $B$. Furthermore, this action is transitive. Hence, if $g \equiv_{\omega_{(5, t)}} \sum_{k=1}^{30} \gamma_{k} q_{t, k}$ with $\gamma_{t} \in \mathbb{F}_{2}$ and $[g]_{\omega_{(5, t)}} \in \Sigma_{5}\left(q_{t, 1}\right)^{\Sigma_{5}}$, then the relations $\tau_{i}(g) \equiv_{\omega_{(5, t)}} g, i=$ $1,2,3,4$, imply $\gamma_{k}=\gamma_{1}, \forall k, 1 \leqslant k \leqslant 30$. Hence, $\Sigma_{5}\left(q_{t, 1}\right)^{\Sigma_{5}}=\left\langle\left[p_{t, 1}\right]_{\omega_{(5, t)}}\right\rangle$ with $p_{t, 1}=\sum_{k=1}^{30} q_{t, k}$.

By a similar computation, we obtain $\Sigma_{5}\left(q_{t, 31}\right)^{\Sigma_{5}}=\left\langle\left[p_{t, 2}\right]_{\omega_{(5, t)}}\right\rangle$ with $p_{t, 2}=\sum_{k=31}^{90} q_{t, k}$, and $\Sigma_{5}\left(q_{t, 91}\right)^{\Sigma_{5}}=\left\langle\left[p_{t, 3}\right]_{\omega_{(5, t)}}\right\rangle$ with $p_{t, 3}=\sum_{k=91}^{110} q_{t, k}$. The lemma follows.

Lemma 4.1.2. We have $\mathcal{M}_{1}^{\Sigma_{5}}=0$ and $\operatorname{dim}\left(\mathcal{M}_{2}^{\Sigma_{5}}\right)=\operatorname{dim}\left(\left(\mathcal{M}_{t, 1}^{*}\right)^{\Sigma_{5}}\right)=1$ for every $t \geqslant 4$.

Proof. Using a result in Section 3, we have $\operatorname{dim}\left(\mathcal{M}_{1}\right)=55$. Suppose $h \equiv_{\omega_{(5, t)}} \sum_{k=91}^{145} \gamma_{k} q_{2, k}$ with $\gamma_{k} \in \mathbb{F}_{2}$ and $[h]_{\omega_{(5, t)}} \in \mathcal{M}_{1}^{\Sigma_{5}}$. By computing $\tau_{j}(h)+h$ in terms of $q_{2, k}, 91 \leqslant k \leqslant 145$ and using the relations $\tau_{j}(h)+h \equiv_{\omega_{(5, t)}} 0,1 \leqslant j \leqslant 4$, we obtain $\gamma_{k}=0$ for all $k, 91 \leqslant k \leqslant 145$.

Now suppose $h \equiv_{\omega_{(5, t)}} \sum_{k=111}^{195} \gamma_{k} q_{3, k}$ and $[h]_{\omega_{(5, t)}} \in \mathcal{M}_{2}^{\Sigma_{5}}$. We compute $\tau_{j}(h)+h$ in terms of $q_{3, k}, 111 \leqslant k \leqslant 195$. By computing directly from the relations $\tau_{j}(h)+h \equiv_{\omega_{(5, t)}} 0, j=1,2,3,4$, one 
gets $\gamma_{k}=0$ for $111 \leqslant k \leqslant 135$ and $\gamma_{k}=\gamma_{136}$ for $136 \leqslant k \leqslant 195$. This implies $\mathcal{M}_{2}^{\Sigma_{5}}=\left\langle\left[p_{3,4}\right]_{\omega_{(5, t)}}\right\rangle$ with $p_{3,4}=\sum_{i=136}^{195} q_{3, i}$.

Similarly, we have $\left(\mathcal{M}_{t, 1}^{*}\right)^{\Sigma_{5}}=\left\langle\left[q_{t, 1}^{*}\right]_{\omega_{(5, t)}}\right\rangle$ with $q_{t, 1}^{*}=\sum_{i=136}^{195} q_{t, i}$ for all $t \geqslant 4$. The lemma is proved.

We now denote by $b_{t, k}=b_{3\left(2^{t}-1\right)+2^{t}, k}$ the admissible monomials in $\left(P_{5}^{+}\right)_{3\left(2^{t}-1\right)+2^{t}}$ as given in Subsection 5.2.

The case $\boldsymbol{t}=\mathbf{2}$. By a direct computation using the results in Section 3, we have the direct summand decomposition of the $\Sigma_{5}$-modules:

$$
Q P_{5}^{+}\left(\omega_{(5,2)}\right)=\Sigma_{5}\left(b_{2,1}\right) \bigoplus \mathcal{M}_{3},
$$

where $\Sigma_{5}\left(b_{2,1}\right)=\left\langle\left[b_{2, k}\right]_{\omega_{(5,2)}}: 1 \leqslant k \leqslant 10\right\rangle$ and $\mathcal{M}_{3}=\left\langle\left[b_{2, k}\right]_{\omega_{(5,2)}}: 11 \leqslant k \leqslant 60\right\rangle$.

The following lemma is proved by a direct computation.

Lemma 4.1.3. $\Sigma_{5}\left(b_{2,1}\right)^{\Sigma_{5}}=0$ and $\mathcal{M}_{3}^{\Sigma_{5}}=\left\langle\left[p_{2,4}:=b_{2,31}+b_{2,32}+\cdots+b_{2,60}\right]_{\omega_{(5,2)}}\right\rangle$.

From Lemmas 4.1.1 and 4.1.3, we obtain the following.

Proposition 4.1.4. $Q P_{5}\left(\omega_{(5,2)}\right)^{\Sigma_{5}}=\left\langle\left[p_{2,1}\right]_{\omega_{(5,2)}},\left[p_{2,2}\right]_{\omega_{(5,2)}},\left[p_{2,4}\right]_{\omega_{(5,2)}}\right\rangle$.

The case $\boldsymbol{t}=3$. By a simple computation, we see that

$$
\begin{aligned}
& \Sigma_{5}\left(b_{3,1}\right)=\left\langle\left[b_{3, k}\right]_{\omega_{(5,3)}}: 1 \leqslant k \leqslant 45\right\rangle, \\
& \Sigma_{5}\left(b_{3,46}\right)=\left\langle\left[b_{3, k}\right]_{\omega_{(5,3)}}: 46 \leqslant k \leqslant 65\right\rangle,
\end{aligned}
$$

and $\mathcal{M}_{4}=\left\langle\left[b_{3, k}\right]_{\omega_{(5,3)}}: 66 \leqslant k \leqslant 260\right\rangle$ are $\Sigma_{5}$-submodules of $Q P_{5}^{+}\left(\omega_{(5,3)}\right)$. Hence, we have a direct summand decomposition of $\Sigma_{5}$-modules:

$$
Q P_{5}^{+}\left(\omega_{(5,3)}\right)=\Sigma_{5}\left(b_{t, 1}\right) \bigoplus \Sigma_{5}\left(b_{t, 46}\right) \bigoplus \mathcal{M}_{4}
$$

Lemma 4.1.5. We have $\operatorname{dim}\left(\Sigma_{5}\left(b_{3,1}\right)^{\Sigma_{5}}\right)=\operatorname{dim}\left(\Sigma_{5}\left(b_{3,46}\right)^{\Sigma_{5}}\right)=1$ and $\mathcal{M}_{4}^{\Sigma_{5}}=0$.

Proof. We have $\operatorname{dim} \Sigma_{5}\left(b_{3,1}\right)=45$ and the set $\left\{\left[b_{3, k}\right]_{\omega_{(5,3)}}: 1 \leqslant k \leqslant 45\right\}$ is a basis of $\Sigma_{5}\left(b_{3,1}\right)$. Suppose $f \equiv_{\omega_{(5,3)}} \sum_{k=1}^{45} \gamma_{k} b_{3, k}$ with $\gamma_{k} \in \mathbb{F}_{2}$ and $[f]_{\omega_{(5,3)}} \in \Sigma_{5}\left(b_{3,1}\right)^{\Sigma_{5}}$. By computing $\tau_{j}(f)+f$ in terms of $b_{3, k}, 1 \leqslant k \leqslant 45$ and using the relations $\tau_{j}(f)+f \equiv_{\omega_{(5,3)}} 0, j=1,2,3,4$, one gets

$$
\begin{aligned}
& \gamma_{1}=\gamma_{k}, \quad \text { for } 2 \leqslant k \leqslant 10 \\
& \gamma_{k}=0, \quad \text { for } 11 \leqslant k \leqslant 45
\end{aligned}
$$

Hence $\Sigma_{5}\left(b_{3,1}\right)^{\Sigma_{5}}=\left\langle\left[p_{3,5}\right]_{\omega_{(5,3)}}\right\rangle$ with $p_{3,5}=\sum_{k=1}^{10} b_{3, k}$. By a similar computation, we get $\Sigma_{5}\left(b_{3,46}\right)^{\Sigma_{5}}=$ $\left\langle\left[p_{3,6}\right]_{\omega_{(5,3)}}\right\rangle$ with $p_{3,6}=\sum_{k=46}^{65} b_{3, k}$ and $\mathcal{M}_{4}^{\Sigma_{5}}=0$. The lemma follows.

Based on Lemmas 4.1.1, 4.1.2 and 4.1.5, one gets the following.

Proposition 4.1.6. $Q P_{5}\left(\omega_{(5,3)}\right)^{\Sigma_{5}}=\left\langle\left[p_{3,1}\right]_{\omega_{(5,3)}},\left[p_{3,2}\right]_{\omega_{(5,3)}}, \ldots,\left[p_{3,6}\right]_{\omega_{(5,3)}}\right\rangle$.

The case $t \geqslant 4$. We set

$$
b_{t, 1}=x_{1} x_{2} x_{3}^{2^{t}-2} x_{4}^{2^{t}-2} x_{5}^{2^{t+1}-1}, \quad b_{t, 46}=x_{1} x_{2}^{2} x_{3}^{2^{t}-4} x_{4}^{2^{t}-1} x_{5}^{2^{t+1}-1} .
$$

Computing from the results in Section 3, one gets

$$
\operatorname{dim}\left(\Sigma_{5}\left(b_{t, 1}\right)\right)=45, \quad \operatorname{dim}\left(\Sigma_{5}\left(b_{t, 46}\right)\right)=20,
$$

where

$$
\begin{aligned}
& \Sigma_{5}\left(b_{t, 1}\right)=\left\langle\left[b_{t, k}\right]_{\omega_{(5, t)}}: 1 \leqslant k \leqslant 45\right\rangle \\
& \Sigma_{5}\left(b_{t, 46}\right)=\left\langle\left[b_{t, k}\right]_{\omega_{(5, t)}}: 46 \leqslant k \leqslant 65\right\rangle
\end{aligned}
$$


Lemma 4.1.7. For any $t \geqslant 4, \Sigma_{5}\left(b_{t, 46}\right)^{\Sigma_{5}}=\left\langle\left[q_{t, 2}^{*}:=b_{t, 46}+b_{t, 47}+\cdots+b_{t, 65}\right]_{\omega_{(5, t)}}\right\rangle$.

The proof of this lemma is straightforward.

For $t \geqslant 4$, by an easy computation, we have a direct summand decomposition of $\Sigma_{5}$-modules:

$$
Q P_{5}^{+}\left(\omega_{(5, t)}\right)=\Sigma_{5}\left(b_{t, 1}\right) \bigoplus \Sigma_{5}\left(b_{t, 46}\right) \bigoplus \mathcal{M}_{t, 2}^{*},
$$

where $\mathcal{M}_{t, 2}^{*}=\left\langle\left[b_{t, k}\right]_{\omega_{(5, t)}}: 66 \leqslant k \leqslant 270\right\rangle$.

Lemma 4.1.8. For any integer $t \geqslant 4$, we have

$$
\Sigma_{5}\left(b_{t, 1}\right)^{\Sigma_{5}}=\left\langle\left[q_{t, 3}^{*}\right]_{\omega_{(5, t)}},\left[q_{t, 4}^{*}\right]_{\omega_{(5, t)}}\right\rangle, \quad\left(\mathcal{M}_{t, 2}^{*}\right)^{\Sigma_{5}}=0
$$

with $q_{t, 3}^{*}:=\sum_{k=1}^{15} b_{t, k}, \quad q_{t, 4}^{*}:=\sum_{k=16}^{45} b_{t, k}$.

Proof. Suppose $g \equiv_{\omega_{(5, t)}} \sum_{k=1}^{45} \gamma_{k} b_{t, k}$ with $\gamma_{k} \in \mathbb{F}_{2}$ and $[g]_{\omega_{(5, t)}} \in \Sigma_{5}\left(b_{t, 1}\right)^{\Sigma_{5}}$. By computing $\tau_{i}(g)+g$ in terms of $b_{t, k}, 1 \leqslant i \leqslant 45$ and using the relations $\tau_{i}(g)+g \equiv_{\omega_{(5, t)}} 0, i=1,2,3,4$, we get

$$
\begin{array}{ll}
\gamma_{1}=\gamma_{k}, & \text { for } 2 \leqslant k \leqslant 15 \\
\gamma_{16}=\gamma_{k}, & \text { for } 17 \leqslant k \leqslant 45
\end{array}
$$

This implies $\Sigma_{5}\left(b_{t, 1}\right)^{\Sigma_{5}}=\left\langle\left[q_{t, 3}^{*}\right],\left[q_{t, 4}^{*}\right]_{\omega_{(5, t)}}\right\rangle$ with $q_{t, 3}^{*}=\sum_{k=1}^{15} b_{t, k}$ and $q_{t, 4}^{*}=\sum_{k=16}^{45} b_{t, k}$.

Now suppose $g \equiv_{\omega_{(5, t)}} \sum_{k=66}^{270} \gamma_{k} b_{t, k}$ and $[g]_{\omega_{(5, t)}} \in\left(\mathcal{M}_{t, 2}^{*}\right)^{\Sigma_{5}}$. We compute $\tau_{j}(g)+g$ in terms of $b_{t, k}, 66 \leqslant k \leqslant 270$. By computing directly from the relations $\tau_{j}(g)+g \equiv_{\omega_{(5, t)}} 0, j=1,2,3,4$, we obtain $\gamma_{k}=0$ for $66 \leqslant k \leqslant 270$. The lemma is proved.

Combining this result and Lemmas 4.1.1, 4.1.2, 4.1.7, 4.1.8, one gets the following.

Proposition 4.1.9. For any $t \geqslant 4$,

$$
Q P_{5}\left(\omega_{(5, t)}\right)^{\Sigma_{5}}=\left\langle\left[p_{t, 1}\right]_{\omega_{(5, t)}},\left[p_{t, 2}\right]_{\omega_{(5, t)}},\left[p_{t, 3}\right]_{\omega_{(5, t)}},\left[q_{t, 1}^{*}\right]_{\omega_{(5, t)}},\left[q_{t, 2}^{*}\right]_{\omega_{(5, t)}},\left[q_{t, 3}^{*}\right]_{\omega_{(5, t)}},\left[q_{t, 4}^{*}\right]_{\omega_{(5, t)}}\right\rangle .
$$

\subsection{Computation of $Q P_{5}\left(\omega_{(5, t)}\right)^{G L_{5}}$}

Let $g \in\left(P_{5}\right)_{3\left(2^{t}-1\right)+2^{t}}$ such that $[g]_{\omega_{(5, t)}} \in\left(Q P_{5}\right)_{3\left(2^{t}-1\right)+2^{t}}^{G L_{5}}$. Then we have $[g]_{\omega_{(5, t)}} \in Q P_{5}\left(\omega_{(5, t)}\right)^{G L_{5}}$.

For $t=1$, we have $3\left(2^{t}-1\right)+2^{t}=5$ and $\left(Q P_{5}\right)_{5}^{G L_{5}}=0$ (see Sum [46, 50]). So, the theorem is true for $t=1$.

For $t=2$, from Proposition 4.1.4, we have $g \equiv_{\omega_{(5,2)}} \gamma_{1} p_{2,1}+\gamma_{2} p_{2,2}+\gamma_{3} p_{2,3}$ with $\gamma_{i} \in \mathbb{F}_{2}, i=1,2,3$. By computing $\tau_{5}(g)+g$ in terms of the admissible monomials, we get

$$
\tau_{5}(g)+g \equiv_{\omega_{(5,2)}}\left(\gamma_{1}+\gamma_{2}\right) x_{1}^{3} x_{2}^{3} x_{4}^{3} x_{5}^{4}+\gamma_{2} x_{1}^{3} x_{2} x_{3}^{4} x_{4}^{3} x_{5}^{2}+\left(\gamma_{2}+\gamma_{3}\right) x_{1} x_{2}^{3} x_{3}^{2} x_{4} x_{5}^{6}+\text { other terms } \equiv 0 .
$$

This relation implies $\gamma_{i}=0$ for $1 \leqslant i \leqslant 3$. The theorem is proved for $t=2$.

For $t=3$, from Proposition 4.1.6, we have $g \equiv_{\omega_{(5,3)}} \sum_{j=1}^{6} \beta_{j} p_{3, j}$ with $\beta_{j} \in \mathbb{F}_{2}, j=1,2, \ldots, 6$. A direct computation shows that

$$
\begin{aligned}
\tau_{5}(g)+g \equiv & \omega_{(5,3)} \beta_{1} x_{1}^{7} x_{2}^{15} x_{5}^{7}+\beta_{2} x_{2} x_{3}^{6} x_{4}^{7} x_{5}^{15}+\left(\beta_{2}+\beta_{3}\right) x_{1} x_{2}^{7} x_{4}^{6} x_{5}^{15}+\left(\beta_{2}+\beta_{4}\right) x_{1} x_{2} x_{3}^{14} x_{4}^{7} x_{5}^{6} \\
& +\beta_{5} x_{1}^{3} x_{2}^{15} x_{3}^{3} x_{4}^{4} x_{5}^{4}+\beta_{6} x_{1}^{7} x_{2}^{7} x_{3}^{9} x_{4}^{2} x_{5}^{4}+\text { other terms } \equiv 0 .
\end{aligned}
$$

From this relation, we obtain $\beta_{j}=0,1 \leqslant j \leqslant 6$. The theorem holds for $t=3$.

For $t \geqslant 4$, by Proposition 4.1.9, we have

$$
g \equiv_{\omega_{(5, t)}} \lambda_{1} p_{t, 1}+\lambda_{2} p_{t, 2}+\lambda_{3} p_{t, 3}+\sum_{j=1}^{4} \lambda_{3+j} q_{t, j}^{*},
$$


where $\lambda_{k} \in \mathbb{F}_{2}, 1 \leqslant k \leqslant 7$. By computing $\tau_{5}(g)+g$ in terms of the admissible monomials, we get

$$
\begin{aligned}
\tau_{5}(g)+g \equiv & \omega_{(5, t)} \lambda_{1} x_{2}^{3} x_{3}^{2^{t}-3} x_{4}^{2^{t}-1} x_{5}^{2^{t+1}-2}+\left(\lambda_{2}+\lambda_{4}\right) x_{1}^{2^{t}-1} x_{3}^{3} x_{4}^{2^{t}-3} x_{5}^{2^{t+1}-2} \\
& +\lambda_{3} x_{1}^{3} x_{2}^{2^{t+1}-3} x_{3}^{2^{t}-1} x_{4}^{2^{t}-2}+\lambda_{4} x_{1}^{7} x_{2}^{2^{t+1}-5} x_{4}^{2^{t}-3} x_{5}^{2^{t}-2} \\
& +\left(\lambda_{1}+\lambda_{2}+\lambda_{5}\right) x_{1}^{3} x_{2}^{5} x_{3}^{2^{t}-6} x_{4}^{2^{t+1}-3} x_{5}^{2^{t}-2} \\
& +\lambda_{6} x_{1}^{3} x_{2}^{5} x_{3}^{2^{t+1}-6} x_{4}^{2^{t}-1} x_{5}^{2^{t}-4}+\lambda_{7} x_{1}^{7} x_{2}^{2^{t}-5} x_{3}^{5} x_{4}^{2^{t}-6} x_{5}^{2^{t+1}-4}+\text { other terms } \equiv 0
\end{aligned}
$$

The last equality implies $\lambda_{k}=0, k=1,2, \ldots, 7$. The theorem is completely proved.

\section{Appendix}

In this section, we list all the admissible monomials of degree $d=3\left(2^{t}-1\right)+2^{t}$ in $P_{5}$.

\subsection{The admissible monomials of degree $3\left(2^{t}-1\right)+2^{t}$ in $P_{5}^{0}$}

Recall that

$$
\mathscr{B}_{5}^{0}\left(3\left(2^{t}-1\right)+2^{t}\right)=\mathscr{B}_{5}^{0}\left(\omega_{(5, t)}\right)=\Phi^{0}\left(\mathscr{B}_{4}\left(3\left(2^{t}-1\right)+2^{t}\right)\right)
$$

with $\omega_{(5, t)}=\underbrace{(3,3, \ldots, 3}_{t \text { times of } 3}, 1)$. By Sum $[47]$, we have

$$
\left|\mathscr{B}_{4}\left(3\left(2^{t}-1\right)+2^{t}\right)\right|= \begin{cases}15 & \text { if } t=1 \\ 35 & \text { if } t=2 \\ 45 & \text { if } t \geqslant 3\end{cases}
$$

Set $u_{t}=\left|\mathscr{B}_{5}^{0}\left(3\left(2^{t}-1\right)+2^{t}\right)\right|$ and $\mathscr{B}_{5}^{0}\left(3\left(2^{t}-1\right)+2^{t}\right)=\left\{q_{t, k}: 1 \leqslant k \leqslant u_{t}\right\}$. We have $u_{1}=45, u_{2}=145, u_{t}=195$ for $t \geqslant 3$.

The admissible monomials $q_{t, k}, 1 \leqslant k \leqslant u_{t}$, are determined as follows:

For $t \geqslant 1$,
1. $x_{3}^{2^{t}-1} x_{4}^{2^{t}-1} x_{5}^{2^{t+1}-1}$
4. $x_{2}^{2^{t}-1} x_{4}^{2^{t}-1} x_{5}^{2^{t+1}-1}$
2. $x_{3}^{2^{t}-1} x_{4}^{2^{t+1}-1} x_{5}^{2^{t}-1}$
5. $x_{2}^{2^{t}-1} x_{4}^{2^{t+1}-1} x_{5}^{2^{t}-1}$
7. $x_{2}^{2^{t}-1} x_{3}^{2^{t}-1} x_{4}^{2^{t+1}-1}$
8. $x_{2}^{2^{t}-1} x_{3}^{2^{t+1}-1} x_{5}^{2^{t}-1}$
10. $x_{2}^{2^{t+1}-1} x_{4}^{2^{t}-1} x_{5}^{2^{t}-1}$
11. $x_{2}^{2^{t+1}-1} x_{3}^{2^{t}-1} x_{5}^{2^{t}-1}$
13. $x_{1}^{2^{t}-1} x_{4}^{2^{t}-1} x_{5}^{2^{t+1}-1}$
14. $x_{1}^{2^{t}-1} x_{4}^{2^{t+1}-1} x_{5}^{2^{t}-1}$
3. $x_{3}^{2^{t+1}-1} x_{4}^{2^{t}-1} x_{5}^{2^{t}-1}$
6. $x_{2}^{2^{t}-1} x_{3}^{2^{t}-1} x_{5}^{2^{t+1}-1}$
9. $x_{2}^{2^{t}-1} x_{3}^{2^{t+1}-1} x_{4}^{2^{t}-1}$
16. $x_{1}^{2^{t}-1} x_{3}^{2^{t}-1} x_{4}^{2^{t+1}-1}$
17. $x_{1}^{2^{t}-1} x_{3}^{2^{t+1}-1} x_{5}^{2^{t}-1}$
12. $x_{2}^{2^{t+1}-1} x_{3}^{2^{t}-1} x_{4}^{2^{t}-1}$
15. $x_{1}^{2^{t}-1} x_{3}^{2^{t}-1} x_{5}^{2^{t+1}-1}$
19. $x_{1}^{2^{t}-1} x_{2}^{2^{t}-1} x_{5}^{2^{t+1}-1}$
20. $x_{1}^{2^{t}-1} x_{2}^{2^{t}-1} x_{4}^{2^{t+1}-1}$
18. $x_{1}^{2^{t}-1} x_{3}^{2^{t+1}-1} x_{4}^{2^{t}-1}$
22. $x_{1}^{2^{t}-1} x_{2}^{2^{t+1}-1} x_{5}^{2^{t}-1}$
23. $x_{1}^{2^{t}-1} x_{2}^{2^{t+1}-1} x_{4}^{2^{t}-1}$
25. $x_{1}^{2^{t+1}-1} x_{4}^{2^{t}-1} x_{5}^{2^{t}-1}$
26. $x_{1}^{2^{t+1}-1} x_{3}^{2^{t}-1} x_{5}^{2^{t}-1}$
28. $x_{1}^{2^{t+1}-1} x_{2}^{2^{t}-1} x_{5}^{2^{t}-1}$
29. $x_{1}^{2^{t+1}-1} x_{2}^{2^{t}-1} x_{4}^{2^{t}-1}$
21. $x_{1}^{2^{t}-1} x_{2}^{2^{t}-1} x_{3}^{2^{t+1}-1}$
24. $x_{1}^{2^{t}-1} x_{2}^{2^{t+1}-1} x_{3}^{2^{t}-1}$
27. $x_{1}^{2^{t+1}-1} x_{3}^{2^{t}-1} x_{4}^{2^{t}-1}$
30. $x_{1}^{2^{t+1}-1} x_{2}^{2^{t}-1} x_{3}^{2^{t}-1}$.

For $t=1$,
31. $x_{2} x_{3} x_{4} x_{5}^{2}$
32. $x_{2} x_{3} x_{4}^{2} x_{5}$
33. $x_{2} x_{3}^{2} x_{4} x_{5}$
34. $x_{1} x_{3} x_{4} x_{5}^{2}$
35. $x_{1} x_{3} x_{4}^{2} x_{5}$
36. $x_{1} x_{3}^{2} x_{4} x_{5}$
37. $x_{1} x_{2} x_{4} x_{5}^{2}$
38. $x_{1} x_{2} x_{4}^{2} x_{5}$
39. $x_{1} x_{2} x_{3} x_{5}^{2}$
40. $x_{1} x_{2} x_{3} x_{4}^{2}$
41. $x_{1} x_{2} x_{3}^{2} x_{5}$
42. $x_{1} x_{2} x_{3}^{2} x_{4}$
43. $x_{1} x_{2}^{2} x_{4} x_{5}$
44. $x_{1} x_{2}^{2} x_{3} x_{5}$
45. $x_{1} x_{2}^{2} x_{3} x_{4}$. 
For $t \geqslant 2$,
31. $x_{1} x_{2}^{2^{t}-2} x_{3}^{2^{t}-1} x_{4}^{2^{t+1}-1}$
34. $x_{1} x_{2}^{2^{t}-1} x_{3}^{2^{t+1}-1} x_{4}^{2^{t}-2}$
37. $x_{1}^{2^{t}-1} x_{2} x_{3}^{2^{t}-2} x_{4}^{2^{t+1}-1}$
32. $x_{1} x_{2}^{2^{t}-2} x_{3}^{2^{t+1}-1} x_{4}^{2^{t}-1}$
35. $x_{1} x_{2}^{2^{t+1}-1} x_{3}^{2^{t}-2} x_{4}^{2^{t}-1}$
40. $x_{1}^{2^{t+1}-1} x_{2} x_{3}^{2^{t}-2} x_{4}^{2^{t}-1}$
38. $x_{1}^{2^{t}-1} x_{2} x_{3}^{2^{t+1}-1} x_{4}^{2^{t}-2}$
41. $x_{1}^{2^{t+1}-1} x_{2} x_{3}^{2^{t}-1} x_{4}^{2^{t}-2}$
33. $x_{1} x_{2}^{2^{t}-1} x_{3}^{2^{t}-2} x_{4}^{2^{t+1}-1}$
36. $x_{1} x_{2}^{2^{t+1}-1} x_{3}^{2^{t}-1} x_{4}^{2^{t}-2}$
39. $x_{1}^{2^{t}-1} x_{2}^{2^{t+1}-1} x_{3} x_{4}^{2^{t}-2}$
43. $x_{2}^{2^{t}-2} x_{3}^{2^{t}-1} x_{4}^{2^{t+1}-1}$
44. $x_{2}^{2^{t}-2} x_{3}^{2^{t+1}-1} x_{4}^{2^{t}-1}$
42. $x_{1}^{2^{t+1}-1} x_{2}^{2^{t}-1} x_{3} x_{4}^{2^{t}-2}$
46. $x_{2}^{2^{t}-1} x_{3}^{2^{t+1}-1} x_{4}^{2^{t}-2}$
47. $x_{2}^{2^{t+1}-1} x_{3}^{2^{t}-2} x_{4}^{2^{t}-1}$
49. $x_{1}^{2^{t}-2} x_{3}^{2^{t}-1} x_{4}^{2^{t+1}-1}$
50. $x_{1}^{2^{t}-2} x_{3}^{2^{t+1}-1} x_{4}^{2^{t}-1}$
45. $x_{2}^{2^{t}-1} x_{3}^{2^{t}-2} x_{4}^{2^{t+1}-1}$
48. $x_{2}^{2^{t+1}-1} x_{3}^{2^{t}-1} x_{4}^{2^{t}-2}$
51. $x_{1}^{2^{t}-2} x_{2}^{2^{t}-1} x_{4}^{2^{t+1}-1}$
52. $x_{1}^{2^{t}-2} x_{2}^{2^{t}-1} x_{3}^{2^{t+1}-1}$
53. $x_{1}^{2^{t}-2} x_{2}^{2^{t+1}-1} x_{4}^{2^{t}-1}$
55. $x_{1}^{2^{t}-1} x_{3}^{2^{t}-2} x_{4}^{2^{t+1}-1}$
56. $x_{1}^{2^{t}-1} x_{3}^{2^{t+1}-1} x_{4}^{2^{t}-2}$
58. $x_{1}^{2^{t}-1} x_{2}^{2^{t}-2} x_{3}^{2^{t+1}-1}$
59. $x_{1}^{2^{t}-1} x_{2}^{2^{t+1}-1} x_{4}^{2^{t}-2}$
61. $x_{1}^{2^{t+1}-1} x_{3}^{2^{t}-2} x_{4}^{2^{t}-1}$
62. $x_{1}^{2^{t+1}-1} x_{3}^{2^{t}-1} x_{4}^{2^{t}-2}$
64. $x_{1}^{2^{t+1}-1} x_{2}^{2^{t}-2} x_{3}^{2^{t}-1}$
65. $x_{1}^{2^{t+1}-1} x_{2}^{2^{t}-1} x_{4}^{2^{t}-2}$
67. $x_{2} x_{3}^{2^{t}-2} x_{4}^{2^{t+1}-1}$
68. $x_{2} x_{3}^{2^{t+1}-1} x_{4}^{2^{t}-2}$
70. $x_{1} x_{3}^{2^{t}-2} x_{4}^{2^{t+1}-1}$
71. $x_{1} x_{3}^{2^{t+1}-1} x_{4}^{2^{t}-2}$
73. $x_{1} x_{2}^{2^{t}-2} x_{3}^{2^{t+1}-1}$
74. $x_{1} x_{2}^{2^{t+1}-1} x_{4}^{2^{t}-2}$
76. $x_{1}^{2^{t+1}-1} x_{3} x_{4}^{2^{t}-2}$
77. $x_{1}^{2^{t+1}-1} x_{2} x_{4}^{2^{t}-2}$
79. $x_{2} x_{3}^{2^{t}-2} x_{4}^{2^{t}-1}$
80. $x_{2} x_{3}^{2^{t}-1} x_{4}^{2^{t}-2}$
82. $x_{1} x_{3}^{2^{t}-2} x_{4}^{2^{t}-1}$
83. $x_{1} x_{3}^{2^{t}-1} x_{4}^{2^{t}-2}$
85. $x_{1} x_{2}^{2^{t}-2} x_{3}^{2^{t}-1}$
86. $x_{1} x_{2}^{2^{t}-1} x_{4}^{2^{t}-2}$
88. $x_{1}^{2^{t}-1} x_{3} x_{4}^{2^{t}-2}$
89. $x_{1}^{2^{t}-1} x_{2} x_{4}^{2^{t}-2}$
54. $x_{1}^{2^{t}-2} x_{2}^{2^{t+1}-1} x_{3}^{2^{t}-1}$
57. $x_{1}^{2^{t}-1} x_{2}^{2^{t}-2} x_{4}^{2^{t+1}-1}$
60. $x_{1}^{2^{t}-1} x_{2}^{2^{t+1}-1} x_{3}^{2^{t}-2}$
63. $x_{1}^{2^{t+1}-1} x_{2}^{2^{t}-2} x_{4}^{2^{t}-1}$
66. $x_{1}^{2^{t+1}-1} x_{2}^{2^{t}-1} x_{3}^{2^{t}-2}$
69. $x_{2}^{2^{t+1}-1} x_{3} x_{4}^{2^{t}-2}$
72. $x_{1} x_{2}^{2^{t}-2} x_{4}^{2^{t+1}-1}$
75. $x_{1} x_{2}^{2^{t+1}-1} x_{3}^{2^{t}-2}$
78. $x_{1}^{2^{t+1}-1} x_{2} x_{3}^{2^{t}-2}$
81. $x_{2}^{2^{t}-1} x_{3} x_{4}^{2^{t}-2}$
84. $x_{1} x_{2}^{2^{t}-2} x_{4}^{2^{t}-1}$
87. $x_{1} x_{2}^{2^{t}-1} x_{3}^{2^{t}-2}$
90. $x_{1}^{2^{t}-1} x_{2} x_{3}^{2^{t}-2}$.

For $t=2$,

\begin{tabular}{|c|c|c|c|c|}
\hline $\begin{array}{l}\text { 1. } x_{2} x_{3}^{3} x_{4}^{3} x_{5}^{6} \\
\text { 6. } x_{2}^{3} x_{3}^{3} x_{4} x_{5}^{6} \\
\text { 01. } x_{1} x_{2}^{3} x_{4}^{6} x_{5}^{3} \\
\text { 06. } x_{1} x_{2}^{6} x_{4}^{3} x_{5}^{3} \\
\text { 11. } x_{1}^{3} x_{3}^{3} x_{4} x_{5}^{6} \\
\text { 16. } x_{1}^{3} x_{2} x_{3}^{6} x_{5}^{3} \\
\text { 21. } x_{2}^{3} x_{3}^{3} x_{4}^{5} x_{5}^{2} \\
\text { 26. } x_{1}^{3} x_{3}^{5} x_{4}^{3} x_{5}^{2} \\
\text { 31. } x_{1}^{3} x_{2}^{5} x_{4}^{3} x_{5}^{2} \\
\text { 36. } x_{2}^{3} x_{3}^{3} x_{4}^{3} x_{5}^{4} \\
\text { 41. } x_{1}^{3} x_{2}^{3} x_{4}^{4} x_{5}^{3}\end{array}$ & $\begin{array}{l}\text { 92. } x_{2} x_{3}^{3} x_{4}^{6} x_{5}^{3} \\
\text { 97. } x_{1} x_{3}^{3} x_{4}^{3} x_{5}^{6} \\
\text { 102. } x_{1} x_{2}^{3} x_{3}^{3} x_{5}^{6} \\
\text { 107. } x_{1} x_{2}^{6} x_{3}^{3} x_{5}^{3} \\
\text { 112. } x_{1}^{3} x_{2} x_{4}^{3} x_{5}^{6} \\
\text { 117. } x_{1}^{3} x_{2} x_{3}^{6} x_{4}^{3} \\
\text { 122. } x_{2}^{3} x_{3}^{5} x_{4}^{2} x_{5}^{3} \\
\text { 127. } x_{1}^{3} x_{2}^{3} x_{4}^{5} x_{5}^{2} \\
\text { 132. } x_{1}^{3} x_{2}^{5} x_{3}^{2} x_{5}^{3} \\
\text { 137. } x_{2}^{3} x_{3}^{3} x_{4}^{4} x_{5}^{3} \\
\text { 142. } x_{1}^{3} x_{2}^{3} x_{3}^{3} x_{5}^{4}\end{array}$ & $\begin{array}{l}\text { 93. } x_{2} x_{3}^{6} x_{4}^{3} x_{5}^{3} \\
\text { 98. } x_{1} x_{3}^{3} x_{4}^{6} x_{5}^{3} \\
\text { 103. } x_{1} x_{2}^{3} x_{3}^{3} x_{4}^{6} \\
\text { 108. } x_{1} x_{2}^{6} x_{3}^{3} x_{4}^{3} \\
\text { 113. } x_{1}^{3} x_{2} x_{4}^{6} x_{5}^{3} \\
\text { 118. } x_{1}^{3} x_{2}^{3} x_{4} x_{5}^{6} \\
\text { 123. } x_{2}^{3} x_{3}^{5} x_{4}^{3} x_{5}^{2} \\
\text { 128. } x_{1}^{3} x_{2}^{3} x_{3}^{5} x_{5}^{2} \\
\text { 133. } x_{1}^{3} x_{2}^{5} x_{3}^{2} x_{4}^{3} \\
\text { 138. } x_{1}^{3} x_{3}^{3} x_{4}^{3} x_{5}^{4} \\
\text { 143. } x_{1}^{3} x_{2}^{3} x_{3}^{3} x_{4}^{4}\end{array}$ & $\begin{array}{l}\text { 94. } x_{2}^{3} x_{3} x_{4}^{3} x_{5}^{6} \\
\text { 99. } x_{1} x_{3}^{6} x_{4}^{3} x_{5}^{3} \\
\text { 104. } x_{1} x_{2}^{3} x_{3}^{6} x_{5}^{3} \\
\text { 109. } x_{1}^{3} x_{3} x_{4}^{3} x_{5}^{6} \\
\text { 114. } x_{1}^{3} x_{2} x_{3}^{3} x_{5}^{6} \\
\text { 119. } x_{1}^{3} x_{2}^{3} x_{3} x_{5}^{6} \\
\text { 124. } x_{1}^{3} x_{3}^{3} x_{4}^{5} x_{5}^{2} \\
\text { 129. } x_{1}^{3} x_{2}^{3} x_{3}^{5} x_{4}^{2} \\
\text { 134. } x_{1}^{3} x_{2}^{5} x_{3}^{3} x_{5}^{2} \\
\text { 139. } x_{1}^{3} x_{3}^{3} x_{4}^{4} x_{5}^{3} \\
\text { 144. } x_{1}^{3} x_{2}^{3} x_{3}^{4} x_{5}^{3}\end{array}$ & $\begin{array}{l}\text { 120. } x_{1}^{3} x_{2}^{3} \\
\text { 125. } x_{1}^{3} x_{3}^{5} \\
\text { 130. } x_{1}^{3} x_{2}^{5} \\
\text { 135. } x_{1}^{3} x_{2}^{5} \\
\text { 140. } x_{1}^{3} x_{2}^{3} \\
\text { 145. } x_{1}^{3} x_{2}^{3}\end{array}$ \\
\hline
\end{tabular}

For $t \geqslant 3$,
91. $x_{2}^{3} x_{3}^{2^{t}-3} x_{4}^{2^{t}-2} x_{5}^{2^{t+1}-1}$
94. $x_{2}^{2^{t+1}-1} x_{3}^{3} x_{4}^{2^{t}-3} x_{5}^{2^{t}-2}$
97. $x_{1}^{3} x_{3}^{2^{t+1}-1} x_{4}^{2^{t}-3} x_{5}^{2^{t}-2}$
100. $x_{1}^{3} x_{2}^{2^{t}-3} x_{3}^{2^{t}-2} x_{5}^{2^{t+1}-1}$
103. $x_{1}^{3} x_{2}^{2^{t}-3} x_{3}^{2^{t+1}-1} x_{4}^{2^{t}-2}$
106. $x_{1}^{3} x_{2}^{2^{t+1}-1} x_{3}^{2^{t}-3} x_{4}^{2^{t}-2}$
109. $x_{1}^{2^{t+1}-1} x_{2}^{3} x_{3}^{2^{t}-3} x_{5}^{2^{t}-2}$

92. $x_{2}^{3} x_{3}^{2^{t}-3} x_{4}^{2^{t+1}-1} x_{5}^{2^{t}-2}$

95. $x_{1}^{3} x_{3}^{2^{t}-3} x_{4}^{2^{t}-2} x_{5}^{2^{t+1}-1}$

98. $x_{1}^{3} x_{2}^{2^{t}-3} x_{4}^{2^{t}-2} x_{5}^{2^{t+1}-1}$

101. $x_{1}^{3} x_{2}^{2^{t}-3} x_{3}^{2^{t}-2} x_{4}^{2^{t+1}-1}$

104. $x_{1}^{3} x_{2}^{2^{t+1}-1} x_{4}^{2^{t}-3} x_{5}^{2^{t}-2}$

107. $x_{1}^{2^{t+1}-1} x_{3}^{3} x_{4}^{2^{t}-3} x_{5}^{2^{t}-2}$

110. $x_{1}^{2^{t+1}-1} x_{2}^{3} x_{3}^{2^{t}-3} x_{4}^{2^{t}-2}$.
93. $x_{2}^{3} x_{3}^{2^{t+1}-1} x_{4}^{2^{t}-3} x_{5}^{2^{t}-2}$

96. $x_{1}^{3} x_{3}^{2^{t}-3} x_{4}^{2^{t+1}-1} x_{5}^{2^{t}-2}$

99. $x_{1}^{3} x_{2}^{2^{t}-3} x_{4}^{2^{t+1}-1} x_{5}^{2^{t}-2}$

102. $x_{1}^{3} x_{2}^{2^{t}-3} x_{3}^{2^{t+1}-1} x_{5}^{2^{t}-2}$

105. $x_{1}^{3} x_{2}^{2^{t+1}-1} x_{3}^{2^{t}-3} x_{5}^{2^{t}-2}$

108. $x_{1}^{2^{t+1}-1} x_{2}^{3} x_{4}^{2^{t}-3} x_{5}^{2^{t}-2}$ 
For $t=3$,

111. $x_{2} x_{3}^{7} x_{4}^{7} x_{5}^{14}$

116. $x_{1} x_{2}^{7} x_{3}^{7} x_{4}^{14}$

121. $x_{2}^{3} x_{3}^{5} x_{4}^{14} x_{5}^{7}$

126. $x_{1}^{3} x_{2}^{5} x_{3}^{14} x_{5}^{7}$

131. $x_{2}^{3} x_{3}^{7} x_{4}^{13} x_{5}^{6}$

136. $x_{2} x_{3}^{7} x_{4}^{14} x_{5}^{7}$

141. $x_{1} x_{3}^{14} x_{4}^{7} x_{5}^{7}$

146. $x_{1} x_{2}^{14} x_{3}^{7} x_{5}^{7}$

151. $x_{1}^{7} x_{2} x_{3}^{14} x_{5}^{7}$

156. $x_{2}^{3} x_{3}^{5} x_{4}^{7} x_{5}^{14}$

161. $x_{1}^{3} x_{2}^{5} x_{3}^{7} x_{4}^{14}$

166. $x_{2}^{3} x_{3}^{13} x_{4}^{6} x_{5}^{7}$

171. $x_{1}^{3} x_{2}^{13} x_{4}^{6} x_{5}^{7}$

176. $x_{1}^{3} x_{2}^{13} x_{3}^{7} x_{4}^{6}$

181. $x_{2}^{7} x_{3}^{11} x_{4}^{5} x_{5}^{6}$

186. $x_{2}^{7} x_{3}^{7} x_{4}^{9} x_{5}^{6}$

191. $x_{2}^{7} x_{3}^{7} x_{4}^{7} x_{5}^{8}$
112. $x_{2}^{7} x_{3} x_{4}^{7} x_{5}^{14}$

117. $x_{1}^{7} x_{3} x_{4}^{7} x_{5}^{14}$

122. $x_{2}^{3} x_{3}^{7} x_{4}^{5} x_{5}^{14}$

127. $x_{1}^{3} x_{2}^{5} x_{3}^{14} x_{4}^{7}$

132. $x_{1}^{3} x_{3}^{7} x_{4}^{13} x_{5}^{6}$

137. $x_{2} x_{3}^{14} x_{4}^{7} x_{5}^{7}$

142. $x_{1} x_{2}^{7} x_{4}^{14} x_{5}^{7}$

147. $x_{1} x_{2}^{14} x_{3}^{7} x_{4}^{7}$

152. $x_{1}^{7} x_{2} x_{3}^{14} x_{4}^{7}$

157. $x_{2}^{7} x_{3}^{3} x_{4}^{5} x_{5}^{14}$

162. $x_{1}^{7} x_{3}^{3} x_{4}^{5} x_{5}^{14}$

167. $x_{2}^{3} x_{3}^{13} x_{4}^{7} x_{5}^{6}$

172. $x_{1}^{3} x_{2}^{13} x_{4}^{7} x_{5}^{6}$

177. $x_{1}^{7} x_{3}^{3} x_{4}^{13} x_{5}^{6}$

182. $x_{1}^{7} x_{3}^{11} x_{4}^{5} x_{5}^{6}$

187. $x_{1}^{7} x_{3}^{7} x_{4}^{9} x_{5}^{6}$

192. $x_{1}^{7} x_{3}^{7} x_{4}^{7} x_{5}^{8}$
113. $x_{1} x_{3}^{7} x_{4}^{7} x_{5}^{14}$

118. $x_{1}^{7} x_{2} x_{4}^{7} x_{5}^{14}$

123. $x_{1}^{3} x_{3}^{5} x_{4}^{14} x_{5}^{7}$

128. $x_{1}^{3} x_{2}^{7} x_{4}^{5} x_{5}^{14}$

133. $x_{1}^{3} x_{2}^{7} x_{4}^{13} x_{5}^{6}$

138. $x_{2}^{7} x_{3} x_{4}^{14} x_{5}^{7}$

143. $x_{1} x_{2}^{7} x_{3}^{14} x_{5}^{7}$

148. $x_{1}^{7} x_{3} x_{4}^{14} x_{5}^{7}$

153. $x_{1}^{7} x_{2}^{7} x_{4} x_{5}^{14}$

158. $x_{1}^{3} x_{3}^{5} x_{4}^{7} x_{5}^{14}$

163. $x_{1}^{7} x_{2}^{3} x_{4}^{5} x_{5}^{14}$

168. $x_{2}^{7} x_{3}^{3} x_{4}^{13} x_{5}^{6}$

173. $x_{1}^{3} x_{2}^{13} x_{3}^{6} x_{5}^{7}$

178. $x_{1}^{7} x_{2}^{3} x_{4}^{13} x_{5}^{6}$

183. $x_{1}^{7} x_{2}^{11} x_{4}^{5} x_{5}^{6}$

188. $x_{1}^{7} x_{2}^{7} x_{4}^{9} x_{5}^{6}$

193. $x_{1}^{7} x_{2}^{7} x_{4}^{7} x_{5}^{8}$
114. $x_{1} x_{2}^{7} x_{4}^{7} x_{5}^{14}$

119. $x_{1}^{7} x_{2} x_{3}^{7} x_{5}^{14}$

124. $x_{1}^{3} x_{3}^{7} x_{4}^{5} x_{5}^{14}$

129. $x_{1}^{3} x_{2}^{7} x_{3}^{5} x_{5}^{14}$

134. $x_{1}^{3} x_{2}^{7} x_{3}^{13} x_{5}^{6}$

139. $x_{2}^{7} x_{3}^{7} x_{4} x_{5}^{14}$

144. $x_{1} x_{2}^{7} x_{3}^{14} x_{4}^{7}$

149. $x_{1}^{7} x_{3}^{7} x_{4} x_{5}^{14}$

154. $x_{1}^{7} x_{2}^{7} x_{3} x_{5}^{14}$

159. $x_{1}^{3} x_{2}^{5} x_{4}^{7} x_{5}^{14}$

164. $x_{1}^{7} x_{2}^{3} x_{3}^{5} x_{5}^{14}$

169. $x_{1}^{3} x_{3}^{13} x_{4}^{6} x_{5}^{7}$

174. $x_{1}^{3} x_{2}^{13} x_{3}^{6} x_{4}^{7}$

179. $x_{1}^{7} x_{2}^{3} x_{3}^{13} x_{5}^{6}$

184. $x_{1}^{7} x_{2}^{11} x_{3}^{5} x_{5}^{6}$

189. $x_{1}^{7} x_{2}^{7} x_{3}^{9} x_{5}^{6}$

194. $x_{1}^{7} x_{2}^{7} x_{3}^{7} x_{5}^{8}$
115. $x_{1} x_{2}^{7} x_{3}^{7} x_{5}^{14}$ 120. $x_{1}^{7} x_{2} x_{3}^{7} x_{4}^{14}$ 125. $x_{1}^{3} x_{2}^{5} x_{4}^{14} x_{5}^{7}$ 130. $x_{1}^{3} x_{2}^{7} x_{3}^{5} x_{4}^{14}$ 135. $x_{1}^{3} x_{2}^{7} x_{3}^{13} x_{4}^{6}$ 140. $x_{1} x_{3}^{7} x_{4}^{14} x_{5}^{7}$ 145. $x_{1} x_{2}^{14} x_{4}^{7} x_{5}^{7}$ 150. $x_{1}^{7} x_{2} x_{4}^{14} x_{5}^{7}$ 155. $x_{1}^{7} x_{2}^{7} x_{3} x_{4}^{14}$ 160. $x_{1}^{3} x_{2}^{5} x_{3}^{7} x_{5}^{14}$ 165. $x_{1}^{7} x_{2}^{3} x_{3}^{5} x_{4}^{14}$ 170. $x_{1}^{3} x_{3}^{13} x_{4}^{7} x_{5}^{6}$ 175. $x_{1}^{3} x_{2}^{13} x_{3}^{7} x_{5}^{6}$ 180. $x_{1}^{7} x_{2}^{3} x_{3}^{13} x_{4}^{6}$ 185. $x_{1}^{7} x_{2}^{11} x_{3}^{5} x_{4}^{6}$ 190. $x_{1}^{7} x_{2}^{7} x_{3}^{9} x_{4}^{6}$ 195. $x_{1}^{7} x_{2}^{7} x_{3}^{7} x_{4}^{8}$.

For $t \geqslant 4$,

111. $x_{2}^{3} x_{3}^{2^{t}-3} x_{4}^{2^{t}-1} x_{5}^{2^{t+1}-2}$

114. $x_{2}^{2^{t}-1} x_{3}^{3} x_{4}^{2^{t}-3} x_{5}^{2^{t+1}-2}$

117. $x_{1}^{3} x_{3}^{2^{t}-1} x_{4}^{2^{t}-3} x_{5}^{2^{t+1}-2}$

120. $x_{1}^{3} x_{2}^{2^{t}-3} x_{3}^{2^{t}-1} x_{5}^{2^{t+1}-2}$

123. $x_{1}^{3} x_{2}^{2^{t}-3} x_{3}^{2^{t+1}-2} x_{4}^{2^{t}-1}$

126. $x_{1}^{3} x_{2}^{2^{t}-1} x_{3}^{2^{t}-3} x_{4}^{2^{t+1}-2}$

129. $x_{1}^{2^{t}-1} x_{2}^{3} x_{3}^{2^{t}-3} x_{5}^{2^{t+1}-2}$

132. $x_{1}^{7} x_{3}^{2^{t}-5} x_{4}^{2^{t+1}-3} x_{5}^{2^{t}-2}$

135. $x_{1}^{7} x_{2}^{2^{t}-5} x_{3}^{2^{t+1}-3} x_{4}^{2^{t}-2}$

138. $x_{2} x_{3}^{2^{t+1}-2} x_{4}^{2^{t}-1} x_{5}^{2^{t}-1}$

141. $x_{2}^{2^{t}-1} x_{3}^{2^{t}-1} x_{4} x_{5}^{2^{t+1}-2}$

144. $x_{1} x_{3}^{2^{t+1}-2} x_{4}^{2^{t}-1} x_{5}^{2^{t}-1}$

147. $x_{1} x_{2}^{2^{t}-1} x_{3}^{2^{t}-1} x_{5}^{2^{t+1}-2}$

150. $x_{1} x_{2}^{2^{t}-1} x_{3}^{2^{t+1}-2} x_{4}^{2^{t}-1}$

153. $x_{1} x_{2}^{2^{t+1}-2} x_{3}^{2^{t}-1} x_{4}^{2^{t}-1}$

156. $x_{1}^{2^{t}-1} x_{3}^{2^{t}-1} x_{4} x_{5}^{2^{t+1}-2}$

159. $x_{1}^{2^{t}-1} x_{2} x_{3}^{2^{t}-1} x_{5}^{2^{t+1}-2}$

162. $x_{1}^{2^{t}-1} x_{2} x_{3}^{2^{t+1}-2} x_{4}^{2^{t}-1}$

165. $x_{1}^{2^{t}-1} x_{2}^{2^{t}-1} x_{3} x_{4}^{2^{t+1}-2}$

168. $x_{2}^{3} x_{3}^{2^{t+1}-3} x_{4}^{2^{t}-1} x_{5}^{2^{t}-2}$

171. $x_{1}^{3} x_{3}^{2^{t+1}-3} x_{4}^{2^{t}-2} x_{5}^{2^{t}-1}$

174. $x_{1}^{3} x_{2}^{2^{t}-1} x_{3}^{2^{t+1}-3} x_{5}^{2^{t}-2}$
112. $x_{2}^{3} x_{3}^{2^{t}-3} x_{4}^{2^{t+1}-2} x_{5}^{2^{t}-1}$

115. $x_{1}^{3} x_{3}^{2^{t}-3} x_{4}^{2^{t}-1} x_{5}^{2^{t+1}-2}$

118. $x_{1}^{3} x_{2}^{2^{t}-3} x_{4}^{2^{t}-1} x_{5}^{2^{t+1}-2}$

121. $x_{1}^{3} x_{2}^{2^{t}-3} x_{3}^{2^{t}-1} x_{4}^{2^{t+1}-2}$

124. $x_{1}^{3} x_{2}^{2^{t}-1} x_{4}^{2^{t}-3} x_{5}^{2^{t+1}-2}$

127. $x_{1}^{2^{t}-1} x_{3}^{3} x_{4}^{2^{t}-3} x_{5}^{2^{t+1}-2}$

130. $x_{1}^{2^{t}-1} x_{2}^{3} x_{3}^{2^{t}-3} x_{4}^{2^{t+1}-2}$

133. $x_{1}^{7} x_{2}^{2^{t}-5} x_{4}^{2^{t+1}-3} x_{5}^{2^{t}-2}$

136. $x_{2} x_{3}^{2^{t}-1} x_{4}^{2^{t}-1} x_{5}^{2^{t+1}-2}$

139. $x_{2}^{2^{t}-1} x_{3} x_{4}^{2^{t}-1} x_{5}^{2^{t+1}-2}$

142. $x_{1} x_{3}^{2^{t}-1} x_{4}^{2^{t}-1} x_{5}^{2^{t+1}-2}$

145. $x_{1} x_{2}^{2^{t}-1} x_{4}^{2^{t}-1} x_{5}^{2^{t+1}-2}$

148. $x_{1} x_{2}^{2^{t}-1} x_{3}^{2^{t}-1} x_{4}^{2^{t+1}-2}$

151. $x_{1} x_{2}^{2^{t+1}-2} x_{4}^{2^{t}-1} x_{5}^{2^{t}-1}$

154. $x_{1}^{2^{t}-1} x_{3} x_{4}^{2^{t}-1} x_{5}^{2^{t+1}-2}$

157. $x_{1}^{2^{t}-1} x_{2} x_{4}^{2^{t}-1} x_{5}^{2^{t+1}-2}$

160. $x_{1}^{2^{t}-1} x_{2} x_{3}^{2^{t}-1} x_{4}^{2^{t+1}-2}$

163. $x_{1}^{2^{t}-1} x_{2}^{2^{t}-1} x_{4} x_{5}^{2^{t+1}-2}$

166. $x_{2}^{3} x_{3}^{2^{t}-1} x_{4}^{2^{t+1}-3} x_{5}^{2^{t}-2}$

169. $x_{2}^{2^{t}-1} x_{3}^{3} x_{4}^{2^{t+1}-3} x_{5}^{2^{t}-2}$

172. $x_{1}^{3} x_{3}^{2^{t+1}-3} x_{4}^{2^{t}-1} x_{5}^{2^{t}-2}$

175. $x_{1}^{3} x_{2}^{2^{t}-1} x_{3}^{2^{t+1}-3} x_{4}^{2^{t}-2}$
113. $x_{2}^{3} x_{3}^{2^{t}-1} x_{4}^{2^{t}-3} x_{5}^{2^{t+1}-2}$

116. $x_{1}^{3} x_{3}^{2^{t}-3} x_{4}^{2^{t+1}-2} x_{5}^{2^{t}-1}$

119. $x_{1}^{3} x_{2}^{2^{t}-3} x_{4}^{2^{t+1}-2} x_{5}^{2^{t}-1}$

122. $x_{1}^{3} x_{2}^{2^{t}-3} x_{3}^{2^{t+1}-2} x_{5}^{2^{t}-1}$

125. $x_{1}^{3} x_{2}^{2^{t}-1} x_{3}^{2^{t}-3} x_{5}^{2^{t+1}-2}$

128. $x_{1}^{2^{t}-1} x_{2}^{3} x_{4}^{2^{t}-3} x_{5}^{2^{t+1}-2}$

131. $x_{2}^{7} x_{3}^{2^{t}-5} x_{4}^{2^{t+1}-3} x_{5}^{2^{t}-2}$

134. $x_{1}^{7} x_{2}^{2^{t}-5} x_{3}^{2^{t+1}-3} x_{5}^{2^{t}-2}$

137. $x_{2} x_{3}^{2^{t}-1} x_{4}^{2^{t+1}-2} x_{5}^{2^{t}-1}$

140. $x_{2}^{2^{t}-1} x_{3} x_{4}^{2^{t+1}-2} x_{5}^{2^{t}-1}$

143. $x_{1} x_{3}^{2^{t}-1} x_{4}^{2^{t+1}-2} x_{5}^{2^{t}-1}$

146. $x_{1} x_{2}^{2^{t}-1} x_{4}^{2^{t+1}-2} x_{5}^{2^{t}-1}$

149. $x_{1} x_{2}^{2^{t}-1} x_{3}^{2^{t+1}-2} x_{5}^{2^{t}-1}$

152. $x_{1} x_{2}^{2^{t+1}-2} x_{3}^{2^{t}-1} x_{5}^{2^{t}-1}$

155. $x_{1}^{2^{t}-1} x_{3} x_{4}^{2^{t+1}-2} x_{5}^{2^{t}-1}$

158. $x_{1}^{2^{t}-1} x_{2} x_{4}^{2^{t+1}-2} x_{5}^{2^{t}-1}$

161. $x_{1}^{2^{t}-1} x_{2} x_{3}^{2^{t+1}-2} x_{5}^{2^{t}-1}$

164. $x_{1}^{2^{t}-1} x_{2}^{2^{t}-1} x_{3} x_{5}^{2^{t+1}-2}$

167. $x_{2}^{3} x_{3}^{2^{t+1}-3} x_{4}^{2^{t}-2} x_{5}^{2^{t}-1}$

170. $x_{1}^{3} x_{3}^{2^{t}-1} x_{4}^{2^{t+1}-3} x_{5}^{2^{t}-2}$

173. $x_{1}^{3} x_{2}^{2^{t}-1} x_{4}^{2^{t+1}-3} x_{5}^{2^{t}-2}$

176. $x_{1}^{3} x_{2}^{2^{t+1}-3} x_{4}^{2^{t}-2} x_{5}^{2^{t}-1}$ 


$\begin{array}{lll}\text { 177. } x_{1}^{3} x_{2}^{2^{t+1}-3} x_{4}^{2^{t}-1} x_{5}^{2^{t}-2} & \text { 178. } x_{1}^{3} x_{2}^{2^{t+1}-3} x_{3}^{2^{t}-2} x_{5}^{2^{t}-1} & \text { 179. } x_{1}^{3} x_{2}^{2^{t+1}-3} x_{3}^{2^{t}-2} x_{4}^{2^{t}-1} \\ \text { 180. } x_{1}^{3} x_{2}^{2^{t+1}-3} x_{3}^{2^{t}-1} x_{5}^{2^{t}-2} & \text { 181. } x_{1}^{3} x_{2}^{2^{t+1}-3} x_{3}^{2^{t}-1} x_{4}^{2^{t}-2} & 182 . x_{1}^{2^{-}-1} x_{3}^{3} x_{4}^{2^{t+1}-3} x_{5}^{2^{t}-2} \\ \text { 183. } x_{1}^{2^{t}-1} x_{2}^{3} x_{4}^{2^{t+1}-3} x_{5}^{2^{t}-2} & \text { 184. } x_{1}^{2^{t}-1} x_{2}^{3} x_{3}^{2^{2+1}-3} x_{5}^{2^{t}-2} & 185 . x_{1}^{2^{t}-1} x_{2}^{3} x_{3}^{2^{t+1}-3} x_{4}^{2^{t}-2} \\ \text { 186. } x_{2}^{7} x_{3}^{2^{t}-5} x_{4}^{2^{t}-3} x_{5}^{2^{t+1}-2} & \text { 187. } x_{1}^{7} x_{3}^{2^{t}-5} x_{4}^{2^{t}-3} x_{5}^{2^{t+1}-2} & \text { 188. } x_{1}^{7} x_{2}^{2^{t}-5} x_{4}^{2^{t}-3} x_{5}^{2^{t+1}-2} \\ \text { 189. } x_{1}^{7} x_{2}^{2^{t}-5} x_{3}^{2^{t}-3} x_{5}^{2^{t+1}-2} & \text { 190. } x_{1}^{7} x_{2}^{2^{t}-5} x_{3}^{2^{t}-3} x_{4}^{2^{t+1}-2} & \text { 191. } x_{2}^{7} x_{3}^{2^{t+1}-5} x_{4}^{2^{t}-3} x_{5}^{2^{t}-2} \\ \text { 192. } x_{1}^{7} x_{3}^{2^{t+1}-5} x_{4}^{2^{t}-3} x_{5}^{2^{t}-2} & \text { 193. } x_{1}^{7} x_{2}^{2^{t+1}-5} x_{4}^{2^{t}-3} x_{5}^{2^{t}-2} & \text { 194. } x_{1}^{7} x_{2}^{2^{t+1}-5} x_{3}^{2^{t}-3} x_{5}^{2^{t}-2} \\ \text { 195. } x_{1}^{7} x_{2}^{2^{t+1}-5} x_{3}^{2^{t}-3} x_{4}^{2^{t}-2} & & \end{array}$

\subsection{The admissible monomials of degree $3\left(2^{t}-1\right)+2^{t}$ in $P_{5}^{+}$}

As it is known,

$$
\mathscr{B}_{5}\left(3\left(2^{t}-1\right)+2^{t}\right)=\mathscr{B}_{5}^{0}\left(3\left(2^{t}-1\right)+2^{t}\right) \bigcup \varphi\left(\mathscr{B}_{5}\left(2^{t+1}-4\right)\right) \bigcup B
$$

where $B=\left(\mathscr{B}_{5}^{+}\left(3\left(2^{t}-1\right)+2^{t}\right) \bigcap \operatorname{Ker}\left(\left(\widetilde{S q}_{*}^{0}\right)_{\left(5,3\left(2^{t}-1\right)+2^{t}\right)}\right)\right)=\mathscr{B}_{5}^{+}\left(\omega_{(5, t)}\right)$, and $\varphi: P_{5} \rightarrow P_{5}, \varphi(y)=x_{1} x_{2} x_{3} x_{4} x_{5} y^{2}, \forall y \in P_{5}$. We have

$$
\left|\mathscr{B}_{5}^{0}\left(3\left(2^{t}-1\right)+2^{t}\right)\right|=\left\{\begin{array}{ll}
45 & \text { if } t=1, \\
145 & \text { if } t=2, \\
195 & \text { if } t \geqslant 3,
\end{array} \quad \text { and }\left|\mathscr{B}_{5}\left(2^{t+1}-4\right)\right|= \begin{cases}1 & \text { if } t=1, \\
45 & \text { if } t=2, \\
190 & \text { if } t=3, \\
480 & \text { if } t=4, \\
650 & \text { if } t=5, \\
651 & \text { if } t \geqslant 6 .\end{cases}\right.
$$

Set $v_{t}=\left|\mathscr{B}_{5}^{+}\left(\omega_{(5, t)}\right)\right|$ and $\mathscr{B}_{5}^{+}\left(\omega_{(5, t)}\right)=\left\{b_{3\left(2^{t}-1\right)+2^{t}, k}: 1 \leqslant k \leqslant v_{t}\right\}$. Then, $v_{1}=1, v_{2}=60, v_{3}=$ $260, v_{t}=270$ for $t \geqslant 4$.

For $t=1$, according to Sum $[46,50], \mathscr{B}_{5}^{+}\left(\omega_{(5,1)}\right)=\left\{b_{5,1}=x_{1} x_{2} x_{3} x_{4} x_{5}\right\}$.

For $t \geqslant 2$, the admissible monomials $b_{3\left(2^{t}-1\right)+2^{t}, k}, 1 \leqslant k \leqslant v_{t}$, are determined as follows:

For $t=2$,
1. $x_{1} x_{2} x_{3}^{2} x_{4}^{2} x_{5}^{7}$
5. $x_{1} x_{2}^{2} x_{3} x_{4}^{7} x_{5}^{2}$
2. $x_{1} x_{2} x_{3}^{2} x_{4}^{7} x_{5}^{2}$
6. $x_{1} x_{2}^{2} x_{3}^{7} x_{4} x_{5}^{2}$
9. $x_{1}^{7} x_{2} x_{3} x_{4}^{2} x_{5}^{2}$
13. $x_{1} x_{2} x_{3}^{3} x_{4}^{2} x_{5}^{6}$
10. $x_{1}^{7} x_{2} x_{3}^{2} x_{4} x_{5}^{2}$
17. $x_{1} x_{2}^{2} x_{3} x_{4}^{3} x_{5}^{6}$
14. $x_{1} x_{2} x_{3}^{3} x_{4}^{6} x_{5}^{2}$
18. $x_{1} x_{2}^{2} x_{3} x_{4}^{6} x_{5}^{3}$
21. $x_{1} x_{2}^{3} x_{3} x_{4}^{6} x_{5}^{2}$
22. $x_{1} x_{2}^{3} x_{3}^{2} x_{4} x_{5}^{6}$
3. $x_{1} x_{2} x_{3}^{7} x_{4}^{2} x_{5}^{2}$
7. $x_{1} x_{2}^{7} x_{3} x_{4}^{2} x_{5}^{2}$
4. $x_{1} x_{2}^{2} x_{3} x_{4}^{2} x_{5}^{7}$
11. $x_{1} x_{2} x_{3}^{2} x_{4}^{3} x_{5}^{6}$
8. $x_{1} x_{2}^{7} x_{3}^{2} x_{4} x_{5}^{2}$
15. $x_{1} x_{2} x_{3}^{6} x_{4}^{2} x_{5}^{3}$
12. $x_{1} x_{2} x_{3}^{2} x_{4}^{6} x_{5}^{3}$
19. $x_{1} x_{2}^{2} x_{3}^{3} x_{4} x_{5}^{6}$
16. $x_{1} x_{2} x_{3}^{6} x_{4}^{3} x_{5}^{2}$
23. $x_{1} x_{2}^{3} x_{3}^{6} x_{4} x_{5}^{2}$
20. $x_{1} x_{2}^{3} x_{3} x_{4}^{2} x_{5}^{6}$
25. $x_{1} x_{2}^{6} x_{3} x_{4}^{3} x_{5}^{2}$
26. $x_{1} x_{2}^{6} x_{3}^{3} x_{4} x_{5}^{2}$
27. $x_{1}^{3} x_{2} x_{3} x_{4}^{2} x_{5}^{6}$
24. $x_{1} x_{2}^{6} x_{3} x_{4}^{2} x_{5}^{3}$
29. $x_{1}^{3} x_{2} x_{3}^{2} x_{4} x_{5}^{6}$
30. $x_{1}^{3} x_{2} x_{3}^{6} x_{4} x_{5}^{2}$
31. $x_{1} x_{2}^{2} x_{3}^{3} x_{4}^{5} x_{5}^{2}$
28. $x_{1}^{3} x_{2} x_{3} x_{4}^{6} x_{5}^{2}$
33. $x_{1} x_{2}^{2} x_{3}^{5} x_{4}^{3} x_{5}^{2}$
34. $x_{1} x_{2}^{3} x_{3}^{2} x_{4}^{5} x_{5}^{2}$
35. $x_{1} x_{2}^{3} x_{3}^{5} x_{4}^{2} x_{5}^{2}$
32. $x_{1} x_{2}^{2} x_{3}^{5} x_{4}^{2} x_{5}^{3}$
37. $x_{1}^{3} x_{2} x_{3}^{5} x_{4}^{2} x_{5}^{2}$
38. $x_{1}^{3} x_{2}^{5} x_{3} x_{4}^{2} x_{5}^{2}$
39. $x_{1}^{3} x_{2}^{5} x_{3}^{2} x_{4} x_{5}^{2}$
36. $x_{1}^{3} x_{2} x_{3}^{2} x_{4}^{5} x_{5}^{2}$
41. $x_{1} x_{2}^{2} x_{3}^{3} x_{4}^{4} x_{5}^{3}$
42. $x_{1} x_{2}^{2} x_{3}^{4} x_{4}^{3} x_{5}^{3}$
43. $x_{1} x_{2}^{3} x_{3}^{2} x_{4}^{3} x_{5}^{4}$
40. $x_{1} x_{2}^{2} x_{3}^{3} x_{4}^{3} x_{5}^{4}$
45. $x_{1} x_{2}^{3} x_{3}^{3} x_{4}^{2} x_{5}^{4}$
46. $x_{1} x_{2}^{3} x_{3}^{3} x_{4}^{4} x_{5}^{2}$
49. $x_{1}^{3} x_{2} x_{3}^{2} x_{4}^{3} x_{5}^{4}$
50. $x_{1}^{3} x_{2} x_{3}^{2} x_{4}^{4} x_{5}^{3}$
47. $x_{1} x_{2}^{3} x_{3}^{4} x_{4}^{2} x_{5}^{3}$
44. $x_{1} x_{2}^{3} x_{3}^{2} x_{4}^{4} x_{5}^{3}$
48. $x_{1} x_{2}^{3} x_{3}^{4} x_{4}^{3} x_{5}^{2}$
53. $x_{1}^{3} x_{2} x_{3}^{4} x_{4}^{2} x_{5}^{3}$
51. $x_{1}^{3} x_{2} x_{3}^{3} x_{4}^{2} x_{5}^{4}$
52. $x_{1}^{3} x_{2} x_{3}^{3} x_{4}^{4} x_{5}^{2}$
54. $x_{1}^{3} x_{2} x_{3}^{4} x_{4}^{3} x_{5}^{2}$
55. $x_{1}^{3} x_{2}^{3} x_{3} x_{4}^{2} x_{5}^{4}$
56. $x_{1}^{3} x_{2}^{3} x_{3} x_{4}^{4} x_{5}^{2}$
57. $x_{1}^{3} x_{2}^{3} x_{3}^{4} x_{4} x_{5}^{2}$
58. $x_{1}^{3} x_{2}^{4} x_{3} x_{4}^{2} x_{5}^{3}$
59. $x_{1}^{3} x_{2}^{4} x_{3} x_{4}^{3} x_{5}^{2}$
60. $x_{1}^{3} x_{2}^{4} x_{3}^{3} x_{4} x_{5}^{2}$. 
For $t=3$,

1. $x_{1} x_{2} x_{3}^{6} x_{4}^{6} x_{5}^{15}$

5. $x_{1}^{3} x_{2}^{3} x_{3}^{4} x_{4}^{4} x_{5}^{15}$

9. $x_{1}^{15} x_{2} x_{3} x_{4}^{6} x_{5}^{6}$

13. $x_{1} x_{2}^{2} x_{3}^{15} x_{4}^{5} x_{5}^{6}$

17. $x_{1} x_{2}^{3} x_{3}^{6} x_{4}^{15} x_{5}^{4}$

21. $x_{1} x_{2}^{6} x_{3} x_{4}^{15} x_{5}^{6}$

25. $x_{1} x_{2}^{15} x_{3}^{3} x_{4}^{6} x_{5}^{4}$

29. $x_{1}^{3} x_{2} x_{3}^{6} x_{4}^{4} x_{5}^{15}$

33. $x_{1}^{3} x_{2}^{5} x_{3}^{2} x_{4}^{4} x_{5}^{15}$

37. $x_{1}^{3} x_{2}^{15} x_{3} x_{4}^{6} x_{5}^{4}$

41. $x_{1}^{15} x_{2} x_{3}^{3} x_{4}^{6} x_{5}^{4}$

45. $x_{1}^{15} x_{2}^{3} x_{3}^{5} x_{4}^{2} x_{5}^{4}$

49. $x_{1} x_{2}^{2} x_{3}^{7} x_{4}^{15} x_{5}^{4}$

53. $x_{1} x_{2}^{7} x_{3}^{2} x_{4}^{15} x_{5}^{4}$

57. $x_{1} x_{2}^{15} x_{3}^{7} x_{4}^{2} x_{5}^{4}$

61. $x_{1}^{7} x_{2}^{15} x_{3} x_{4}^{2} x_{5}^{4}$

65. $x_{1}^{15} x_{2}^{7} x_{3} x_{4}^{2} x_{5}^{4}$

69. $x_{1} x_{2} x_{3}^{7} x_{4}^{14} x_{5}^{6}$

73. $x_{1} x_{2}^{2} x_{3}^{5} x_{4}^{14} x_{5}^{7}$

77. $x_{1} x_{2}^{2} x_{3}^{7} x_{4}^{13} x_{5}^{6}$

81. $x_{1} x_{2}^{3} x_{3}^{4} x_{4}^{7} x_{5}^{14}$

85. $x_{1} x_{2}^{3} x_{3}^{6} x_{4}^{5} x_{5}^{14}$

89. $x_{1} x_{2}^{3} x_{3}^{7} x_{4}^{4} x_{5}^{14}$

93. $x_{1} x_{2}^{3} x_{3}^{12} x_{4}^{6} x_{5}^{7}$

97. $x_{1} x_{2}^{3} x_{3}^{14} x_{4}^{5} x_{5}^{6}$

101. $x_{1} x_{2}^{6} x_{3}^{3} x_{4}^{5} x_{5}^{14}$

105. $x_{1} x_{2}^{6} x_{3}^{7} x_{4}^{9} x_{5}^{6}$

109. $x_{1} x_{2}^{7} x_{3}^{2} x_{4}^{5} x_{5}^{14}$

113. $x_{1} x_{2}^{7} x_{3}^{3} x_{4}^{4} x_{5}^{14}$

117. $x_{1} x_{2}^{7} x_{3}^{6} x_{4} x_{5}^{14}$

121. $x_{1} x_{2}^{7} x_{3}^{7} x_{4}^{6} x_{5}^{8}$

125. $x_{1} x_{2}^{7} x_{3}^{11} x_{4}^{4} x_{5}^{6}$

129. $x_{1} x_{2}^{14} x_{3} x_{4}^{7} x_{5}^{6}$

133. $x_{1}^{3} x_{2} x_{3}^{4} x_{4}^{14} x_{5}^{7}$

137. $x_{1}^{3} x_{2} x_{3}^{6} x_{4}^{7} x_{5}^{12}$

141. $x_{1}^{3} x_{2} x_{3}^{7} x_{4}^{6} x_{5}^{12}$

145. $x_{1}^{3} x_{2} x_{3}^{12} x_{4}^{7} x_{5}^{6}$

149. $x_{1}^{3} x_{2} x_{3}^{14} x_{4}^{7} x_{5}^{4}$

153. $x_{1}^{3} x_{2}^{3} x_{3}^{5} x_{4}^{4} x_{5}^{14}$

157. $x_{1}^{3} x_{2}^{3} x_{3}^{7} x_{4}^{4} x_{5}^{12}$

161. $x_{1}^{3} x_{2}^{3} x_{3}^{13} x_{4}^{6} x_{5}^{4}$

165. $x_{1}^{3} x_{2}^{5} x_{3} x_{4}^{6} x_{5}^{14}$

169. $x_{1}^{3} x_{2}^{5} x_{3}^{2} x_{4}^{12} x_{5}^{7}$

173. $x_{1}^{3} x_{2}^{5} x_{3}^{3} x_{4}^{12} x_{5}^{6}$

177. $x_{1}^{3} x_{2}^{5} x_{3}^{6} x_{4}^{8} x_{5}^{7}$

181. $x_{1}^{3} x_{2}^{5} x_{3}^{7} x_{4}^{8} x_{5}^{6}$
2. $x_{1} x_{2} x_{3}^{6} x_{4}^{15} x_{5}^{6}$

6. $x_{1}^{3} x_{2}^{3} x_{3}^{4} x_{4}^{15} x_{5}^{4}$

10. $x_{1}^{15} x_{2}^{3} x_{3}^{3} x_{4}^{4} x_{5}^{4}$

14. $x_{1} x_{2}^{3} x_{3}^{4} x_{4}^{6} x_{5}^{15}$

18. $x_{1} x_{2}^{3} x_{3}^{15} x_{4}^{4} x_{5}^{6}$

22. $x_{1} x_{2}^{6} x_{3}^{15} x_{4} x_{5}^{6}$

26. $x_{1} x_{2}^{15} x_{3}^{6} x_{4} x_{5}^{6}$

30. $x_{1}^{3} x_{2} x_{3}^{6} x_{4}^{15} x_{5}^{4}$

34. $x_{1}^{3} x_{2}^{5} x_{3}^{2} x_{4}^{15} x_{5}^{4}$

38. $x_{1}^{3} x_{2}^{15} x_{3}^{5} x_{4}^{2} x_{5}^{4}$

42. $x_{1}^{15} x_{2} x_{3}^{6} x_{4} x_{5}^{6}$

46. $x_{1} x_{2}^{2} x_{3}^{4} x_{4}^{7} x_{5}^{15}$

50. $x_{1} x_{2}^{2} x_{3}^{15} x_{4}^{4} x_{5}^{7}$

54. $x_{1} x_{2}^{7} x_{3}^{15} x_{4}^{2} x_{5}^{4}$

58. $x_{1}^{7} x_{2} x_{3}^{2} x_{4}^{4} x_{5}^{15}$

62. $x_{1}^{15} x_{2} x_{3}^{2} x_{4}^{4} x_{5}^{7}$

66. $x_{1} x_{2} x_{3}^{6} x_{4}^{7} x_{5}^{14}$

70. $x_{1} x_{2} x_{3}^{14} x_{4}^{6} x_{5}^{7}$

74. $x_{1} x_{2}^{2} x_{3}^{7} x_{4}^{5} x_{5}^{14}$

78. $x_{1} x_{2}^{2} x_{3}^{12} x_{4}^{7} x_{5}^{7}$

82. $x_{1} x_{2}^{3} x_{3}^{4} x_{4}^{14} x_{5}^{7}$

86. $x_{1} x_{2}^{3} x_{3}^{6} x_{4}^{7} x_{5}^{12}$

90. $x_{1} x_{2}^{3} x_{3}^{7} x_{4}^{6} x_{5}^{12}$

94. $x_{1} x_{2}^{3} x_{3}^{12} x_{4}^{7} x_{5}^{6}$

98. $x_{1} x_{2}^{3} x_{3}^{14} x_{4}^{7} x_{5}^{4}$

102. $x_{1} x_{2}^{6} x_{3}^{3} x_{4}^{13} x_{5}^{6}$

106. $x_{1} x_{2}^{6} x_{3}^{11} x_{4}^{5} x_{5}^{6}$

110. $x_{1} x_{2}^{7} x_{3}^{2} x_{4}^{7} x_{5}^{12}$

114. $x_{1} x_{2}^{7} x_{3}^{3} x_{4}^{6} x_{5}^{12}$

118. $x_{1} x_{2}^{7} x_{3}^{6} x_{4}^{7} x_{5}^{8}$

122. $x_{1} x_{2}^{7} x_{3}^{7} x_{4}^{8} x_{5}^{6}$

126. $x_{1} x_{2}^{7} x_{3}^{11} x_{4}^{6} x_{5}^{4}$

130. $x_{1} x_{2}^{14} x_{3}^{3} x_{4}^{5} x_{5}^{6}$

134. $x_{1}^{3} x_{2} x_{3}^{5} x_{4}^{6} x_{5}^{14}$

138. $x_{1}^{3} x_{2} x_{3}^{6} x_{4}^{12} x_{5}^{7}$

142. $x_{1}^{3} x_{2} x_{3}^{7} x_{4}^{12} x_{5}^{6}$

146. $x_{1}^{3} x_{2} x_{3}^{13} x_{4}^{6} x_{5}^{6}$

150. $x_{1}^{3} x_{2}^{3} x_{3}^{4} x_{4}^{5} x_{5}^{14}$

154. $x_{1}^{3} x_{2}^{3} x_{3}^{5} x_{4}^{6} x_{5}^{12}$

158. $x_{1}^{3} x_{2}^{3} x_{3}^{7} x_{4}^{12} x_{5}^{4}$

162. $x_{1}^{3} x_{2}^{4} x_{3}^{3} x_{4}^{5} x_{5}^{14}$

166. $x_{1}^{3} x_{2}^{5} x_{3} x_{4}^{14} x_{5}^{6}$

170. $x_{1}^{3} x_{2}^{5} x_{3}^{2} x_{4}^{13} x_{5}^{6}$

174. $x_{1}^{3} x_{2}^{5} x_{3}^{3} x_{4}^{14} x_{5}^{4}$

178. $x_{1}^{3} x_{2}^{5} x_{3}^{6} x_{4}^{9} x_{5}^{6}$

182. $x_{1}^{3} x_{2}^{5} x_{3}^{7} x_{4}^{10} x_{5}^{4}$
3. $x_{1} x_{2} x_{3}^{15} x_{4}^{6} x_{5}^{6}$

7. $x_{1}^{3} x_{2}^{3} x_{3}^{15} x_{4}^{4} x_{5}^{4}$

11. $x_{1} x_{2}^{2} x_{3}^{5} x_{4}^{6} x_{5}^{15}$

15. $x_{1} x_{2}^{3} x_{3}^{4} x_{4}^{15} x_{5}^{6}$

19. $x_{1} x_{2}^{3} x_{3}^{15} x_{4}^{6} x_{5}^{4}$

23. $x_{1} x_{2}^{15} x_{3}^{2} x_{4}^{5} x_{5}^{6}$

27. $x_{1}^{3} x_{2} x_{3}^{4} x_{4}^{6} x_{5}^{15}$

31. $x_{1}^{3} x_{2} x_{3}^{15} x_{4}^{4} x_{5}^{6}$

35. $x_{1}^{3} x_{2}^{5} x_{3}^{15} x_{4}^{2} x_{5}^{4}$

39. $x_{1}^{15} x_{2} x_{3}^{2} x_{4}^{5} x_{5}^{6}$

43. $x_{1}^{15} x_{2}^{3} x_{3} x_{4}^{4} x_{5}^{6}$

47. $x_{1} x_{2}^{2} x_{3}^{4} x_{4}^{15} x_{5}^{7}$

51. $x_{1} x_{2}^{2} x_{3}^{15} x_{4}^{7} x_{5}^{4}$

55. $x_{1} x_{2}^{15} x_{3}^{2} x_{4}^{4} x_{5}^{7}$

59. $x_{1}^{7} x_{2} x_{3}^{2} x_{4}^{15} x_{5}^{4}$

63. $x_{1}^{15} x_{2} x_{3}^{2} x_{4}^{7} x_{5}^{4}$

67. $x_{1} x_{2} x_{3}^{6} x_{4}^{14} x_{5}^{7}$

71. $x_{1} x_{2} x_{3}^{14} x_{4}^{7} x_{5}^{6}$

75. $x_{1} x_{2}^{2} x_{3}^{7} x_{4}^{7} x_{5}^{12}$

79. $x_{1} x_{2}^{2} x_{3}^{13} x_{4}^{6} x_{5}^{7}$

83. $x_{1} x_{2}^{3} x_{3}^{5} x_{4}^{6} x_{5}^{14}$,

87. $x_{1} x_{2}^{3} x_{3}^{6} x_{4}^{12} x_{5}^{7}$

91. $x_{1} x_{2}^{3} x_{3}^{7} x_{4}^{12} x_{5}^{6}$

95. $x_{1} x_{2}^{3} x_{3}^{13} x_{4}^{6} x_{5}^{6}$

99. $x_{1} x_{2}^{6} x_{3} x_{4}^{7} x_{5}^{14}$

103. $x_{1} x_{2}^{6} x_{3}^{7} x_{4} x_{5}^{14}$

107. $x_{1} x_{2}^{7} x_{3} x_{4}^{6} x_{5}^{14}$

111. $x_{1} x_{2}^{7} x_{3}^{2} x_{4}^{12} x_{5}^{7}$

115. $x_{1} x_{2}^{7} x_{3}^{3} x_{4}^{12} x_{5}^{6}$

119. $x_{1} x_{2}^{7} x_{3}^{6} x_{4}^{9} x_{5}^{6}$

123. $x_{1} x_{2}^{7} x_{3}^{7} x_{4}^{10} x_{5}^{4}$

127. $x_{1} x_{2}^{7} x_{3}^{14} x_{4} x_{5}^{6}$

131. $x_{1} x_{2}^{14} x_{3}^{7} x_{4} x_{5}^{6}$

135. $x_{1}^{3} x_{2} x_{3}^{5} x_{4}^{14} x_{5}^{6}$

139. $x_{1}^{3} x_{2} x_{3}^{6} x_{4}^{13} x_{5}^{6}$

143. $x_{1}^{3} x_{2} x_{3}^{7} x_{4}^{14} x_{5}^{4}$

147. $x_{1}^{3} x_{2} x_{3}^{14} x_{4}^{4} x_{5}^{7}$

151. $x_{1}^{3} x_{2}^{3} x_{3}^{4} x_{4}^{7} x_{5}^{12}$

155. $x_{1}^{3} x_{2}^{3} x_{3}^{5} x_{4}^{12} x_{5}^{6}$

159. $x_{1}^{3} x_{2}^{3} x_{3}^{12} x_{4}^{5} x_{5}^{6}$

163. $x_{1}^{3} x_{2}^{4} x_{3}^{3} x_{4}^{13} x_{5}^{6}$

167. $x_{1}^{3} x_{2}^{5} x_{3}^{2} x_{4}^{5} x_{5}^{14}$

171. $x_{1}^{3} x_{2}^{5} x_{3}^{3} x_{4}^{4} x_{5}^{14}$

175. $x_{1}^{3} x_{2}^{5} x_{3}^{6} x_{4} x_{5}^{14}$

179. $x_{1}^{3} x_{2}^{5} x_{3}^{7} x_{4}^{2} x_{5}^{12}$

183. $x_{1}^{3} x_{2}^{5} x_{3}^{10} x_{4}^{4} x_{5}^{7}$
4. $x_{1} x_{2}^{15} x_{3} x_{4}^{6} x_{5}^{6}$

8. $x_{1}^{3} x_{2}^{15} x_{3}^{3} x_{4}^{4} x_{5}^{4}$

12. $x_{1} x_{2}^{2} x_{3}^{5} x_{4}^{15} x_{5}^{6}$

16. $x_{1} x_{2}^{3} x_{3}^{6} x_{4}^{4} x_{5}^{15}$

20. $x_{1} x_{2}^{6} x_{3} x_{4}^{6} x_{5}^{15}$

24. $x_{1} x_{2}^{15} x_{3}^{3} x_{4}^{4} x_{5}^{6}$

28. $x_{1}^{3} x_{2} x_{3}^{4} x_{4}^{15} x_{5}^{6}$

32. $x_{1}^{3} x_{2} x_{3}^{15} x_{4}^{6} x_{5}^{4}$

36. $x_{1}^{3} x_{2}^{15} x_{3} x_{4}^{4} x_{5}^{6}$

40. $x_{1}^{15} x_{2} x_{3}^{3} x_{4}^{4} x_{5}^{6}$

44. $x_{1}^{15} x_{2}^{3} x_{3} x_{4}^{6} x_{5}^{4}$

48. $x_{1} x_{2}^{2} x_{3}^{7} x_{4}^{4} x_{5}^{15}$

52. $x_{1} x_{2}^{7} x_{3}^{2} x_{4}^{4} x_{5}^{15}$

56. $x_{1} x_{2}^{15} x_{3}^{2} x_{4}^{7} x_{5}^{4}$

60. $x_{1}^{7} x_{2} x_{3}^{15} x_{4}^{2} x_{5}^{4}$

64. $x_{1}^{15} x_{2} x_{3}^{7} x_{4}^{2} x_{5}^{4}$

68. $x_{1} x_{2} x_{3}^{7} x_{4}^{6} x_{5}^{14}$

72. $x_{1} x_{2}^{2} x_{3}^{5} x_{4}^{7} x_{5}^{14}$

76. $x_{1} x_{2}^{2} x_{3}^{7} x_{4}^{12} x_{5}^{7}$

80. $x_{1} x_{2}^{2} x_{3}^{13} x_{4}^{7} x_{5}^{6}$

84. $x_{1} x_{2}^{3} x_{3}^{5} x_{4}^{14} x_{5}^{6}$

88. $x_{1} x_{2}^{3} x_{3}^{6} x_{4}^{13} x_{5}^{6}$

92. $x_{1} x_{2}^{3} x_{3}^{7} x_{4}^{14} x_{5}^{4}$

96. $x_{1} x_{2}^{3} x_{3}^{14} x_{4}^{4} x_{5}^{7}$

100. $x_{1} x_{2}^{6} x_{3} x_{4}^{14} x_{5}^{7}$

104. $x_{1} x_{2}^{6} x_{3}^{7} x_{4}^{7} x_{5}^{8}$

108. $x_{1} x_{2}^{7} x_{3} x_{4}^{14} x_{5}^{6}$

112. $x_{1} x_{2}^{7} x_{3}^{2} x_{4}^{13} x_{5}^{6}$

116. $x_{1} x_{2}^{7} x_{3}^{3} x_{4}^{14} x_{5}^{4}$

120. $x_{1} x_{2}^{7} x_{3}^{7} x_{4}^{2} x_{5}^{12}$

124. $x_{1} x_{2}^{7} x_{3}^{10} x_{4}^{5} x_{5}^{6}$

128. $x_{1} x_{2}^{14} x_{3} x_{4}^{6} x_{5}^{7}$

132. $x_{1}^{3} x_{2} x_{3}^{4} x_{4}^{7} x_{5}^{14}$

136. $x_{1}^{3} x_{2} x_{3}^{6} x_{4}^{5} x_{5}^{14}$

140. $x_{1}^{3} x_{2} x_{3}^{7} x_{4}^{4} x_{5}^{14}$

144. $x_{1}^{3} x_{2} x_{3}^{12} x_{4}^{6} x_{5}^{7}$

148. $x_{1}^{3} x_{2} x_{3}^{14} x_{4}^{5} x_{5}^{6}$

152. $x_{1}^{3} x_{2}^{3} x_{3}^{4} x_{4}^{13} x_{5}^{6}$

156. $x_{1}^{3} x_{2}^{3} x_{3}^{5} x_{4}^{14} x_{5}^{4}$

160. $x_{1}^{3} x_{2}^{3} x_{3}^{13} x_{4}^{4} x_{5}^{6}$

164. $x_{1}^{3} x_{2}^{4} x_{3}^{11} x_{4}^{5} x_{5}^{6}$

168. $x_{1}^{3} x_{2}^{5} x_{3}^{2} x_{4}^{7} x_{5}^{12}$

172. $x_{1}^{3} x_{2}^{5} x_{3}^{3} x_{4}^{6} x_{5}^{12}$

176. $x_{1}^{3} x_{2}^{5} x_{3}^{6} x_{4}^{7} x_{5}^{8}$

180. $x_{1}^{3} x_{2}^{5} x_{3}^{7} x_{4}^{6} x_{5}^{8}$

184. $x_{1}^{3} x_{2}^{5} x_{3}^{10} x_{4}^{5} x_{5}^{6}$ 
185. $x_{1}^{3} x_{2}^{5} x_{3}^{10} x_{4}^{7} x_{5}^{4}$ 189. $x_{1}^{3} x_{2}^{7} x_{3} x_{4}^{4} x_{5}^{14}$ 193. $x_{1}^{3} x_{2}^{7} x_{3}^{3} x_{4}^{4} x_{5}^{12}$ 197. $x_{1}^{3} x_{2}^{7} x_{3}^{5} x_{4}^{8} x_{5}^{6}$ 201. $x_{1}^{3} x_{2}^{7} x_{3}^{9} x_{4}^{6} x_{5}^{4}$ 205. $x_{1}^{3} x_{2}^{13} x_{3}^{2} x_{4}^{4} x_{5}^{7}$ 209. $x_{1}^{3} x_{2}^{13} x_{3}^{3} x_{4}^{6} x_{5}^{4}$ 213. $x_{1}^{7} x_{2} x_{3} x_{4}^{14} x_{5}^{6}$ 217. $x_{1}^{7} x_{2} x_{3}^{2} x_{4}^{13} x_{5}^{6}$ 221. $x_{1}^{7} x_{2} x_{3}^{3} x_{4}^{14} x_{5}^{4}$ 225. $x_{1}^{7} x_{2} x_{3}^{7} x_{4}^{2} x_{5}^{12}$ 229. $x_{1}^{7} x_{2} x_{3}^{10} x_{4}^{5} x_{5}^{6}$ 233. $x_{1}^{7} x_{2}^{3} x_{3} x_{4}^{4} x_{5}^{14}$ 237. $x_{1}^{7} x_{2}^{3} x_{3}^{3} x_{4}^{4} x_{5}^{12}$ 241. $x_{1}^{7} x_{2}^{3} x_{3}^{5} x_{4}^{8} x_{5}^{6}$ 245. $x_{1}^{7} x_{2}^{3} x_{3}^{9} x_{4}^{6} x_{5}^{4}$ 249. $x_{1}^{7} x_{2}^{7} x_{3} x_{4}^{6} x_{5}^{8}$ 253. $x_{1}^{7} x_{2}^{7} x_{3}^{9} x_{4}^{2} x_{5}^{4}$ 257. $x_{1}^{7} x_{2}^{11} x_{3} x_{4}^{4} x_{5}^{6}$
186. $x_{1}^{3} x_{2}^{5} x_{3}^{11} x_{4}^{4} x_{5}^{6}$ 190. $x_{1}^{3} x_{2}^{7} x_{3} x_{4}^{6} x_{5}^{12}$ 194. $x_{1}^{3} x_{2}^{7} x_{3}^{3} x_{4}^{12} x_{5}^{4}$ 198. $x_{1}^{3} x_{2}^{7} x_{3}^{5} x_{4}^{10} x_{5}^{4}$ 202. $x_{1}^{3} x_{2}^{7} x_{3}^{11} x_{4}^{4} x_{5}^{4}$ 206. $x_{1}^{3} x_{2}^{13} x_{3}^{2} x_{4}^{5} x_{5}^{6}$ 210. $x_{1}^{3} x_{2}^{13} x_{3}^{6} x_{4} x_{5}^{6}$ 214. $x_{1}^{7} x_{2} x_{3}^{2} x_{4}^{5} x_{5}^{14}$ 218. $x_{1}^{7} x_{2} x_{3}^{3} x_{4}^{4} x_{5}^{14}$ 222. $x_{1}^{7} x_{2} x_{3}^{6} x_{4} x_{5}^{14}$ 226. $x_{1}^{7} x_{2} x_{3}^{7} x_{4}^{6} x_{5}^{8}$ 230. $x_{1}^{7} x_{2} x_{3}^{11} x_{4}^{4} x_{5}^{6}$ 234. $x_{1}^{7} x_{2}^{3} x_{3} x_{4}^{6} x_{5}^{12}$ 238. $x_{1}^{7} x_{2}^{3} x_{3}^{3} x_{4}^{12} x_{5}^{4}$ 242. $x_{1}^{7} x_{2}^{3} x_{3}^{5} x_{4}^{10} x_{5}^{4}$ 246. $x_{1}^{7} x_{2}^{3} x_{3}^{11} x_{4}^{4} x_{5}^{4}$ 250. $x_{1}^{7} x_{2}^{7} x_{3} x_{4}^{8} x_{5}^{6}$ 254. $x_{1}^{7} x_{2}^{9} x_{3}^{2} x_{4}^{5} x_{5}^{6}$ 258. $x_{1}^{7} x_{2}^{11} x_{3} x_{4}^{6} x_{5}^{4}$
187. $x_{1}^{3} x_{2}^{5} x_{3}^{11} x_{4}^{6} x_{5}^{4}$ 191. $x_{1}^{3} x_{2}^{7} x_{3} x_{4}^{12} x_{5}^{6}$ 195. $x_{1}^{3} x_{2}^{7} x_{3}^{5} x_{4}^{2} x_{5}^{12}$ 199. $x_{1}^{3} x_{2}^{7} x_{3}^{8} x_{4}^{5} x_{5}^{6}$ 203. $x_{1}^{3} x_{2}^{7} x_{3}^{13} x_{4}^{2} x_{5}^{4}$ 207. $x_{1}^{3} x_{2}^{13} x_{3}^{2} x_{4}^{7} x_{5}^{4}$ 211. $x_{1}^{3} x_{2}^{13} x_{3}^{7} x_{4}^{2} x_{5}^{4}$ 215. $x_{1}^{7} x_{2} x_{3}^{2} x_{4}^{7} x_{5}^{12}$ 219. $x_{1}^{7} x_{2} x_{3}^{3} x_{4}^{6} x_{5}^{12}$ 223. $x_{1}^{7} x_{2} x_{3}^{6} x_{4}^{7} x_{5}^{8}$ 227. $x_{1}^{7} x_{2} x_{3}^{7} x_{4}^{8} x_{5}^{6}$ 231. $x_{1}^{7} x_{2} x_{3}^{11} x_{4}^{6} x_{5}^{4}$ 235. $x_{1}^{7} x_{2}^{3} x_{3} x_{4}^{12} x_{5}^{6}$ 239. $x_{1}^{7} x_{2}^{3} x_{3}^{5} x_{4}^{2} x_{5}^{12}$ 243. $x_{1}^{7} x_{2}^{3} x_{3}^{8} x_{4}^{5} x_{5}^{6}$ 247. $x_{1}^{7} x_{2}^{3} x_{3}^{13} x_{4}^{2} x_{5}^{4}$ 251. $x_{1}^{7} x_{2}^{7} x_{3} x_{4}^{10} x_{5}^{4}$ 255. $x_{1}^{7} x_{2}^{9} x_{3}^{3} x_{4}^{4} x_{5}^{6}$ 259. $x_{1}^{7} x_{2}^{11} x_{3}^{3} x_{4}^{4} x_{5}^{4}$
188. $x_{1}^{3} x_{2}^{5} x_{3}^{14} x_{4} x_{5}^{6}$ 192. $x_{1}^{3} x_{2}^{7} x_{3} x_{4}^{14} x_{5}^{4}$ 196. $x_{1}^{3} x_{2}^{7} x_{3}^{5} x_{4}^{6} x_{5}^{8}$ 200. $x_{1}^{3} x_{2}^{7} x_{3}^{9} x_{4}^{4} x_{5}^{6}$ 204. $x_{1}^{3} x_{2}^{13} x_{3} x_{4}^{6} x_{5}^{6}$ 208. $x_{1}^{3} x_{2}^{13} x_{3}^{3} x_{4}^{4} x_{5}^{6}$ 212. $x_{1}^{7} x_{2} x_{3} x_{4}^{6} x_{5}^{14}$ 216. $x_{1}^{7} x_{2} x_{3}^{2} x_{4}^{12} x_{5}^{7}$ 220. $x_{1}^{7} x_{2} x_{3}^{3} x_{4}^{12} x_{5}^{6}$ 224. $x_{1}^{7} x_{2} x_{3}^{6} x_{4}^{9} x_{5}^{6}$ 228. $x_{1}^{7} x_{2} x_{3}^{7} x_{4}^{10} x_{5}^{4}$ 232. $x_{1}^{7} x_{2} x_{3}^{14} x_{4} x_{5}^{6}$ 236. $x_{1}^{7} x_{2}^{3} x_{3} x_{4}^{14} x_{5}^{4}$ 240. $x_{1}^{7} x_{2}^{3} x_{3}^{5} x_{4}^{6} x_{5}^{8}$ 244. $x_{1}^{7} x_{2}^{3} x_{3}^{9} x_{4}^{4} x_{5}^{6}$ 248. $x_{1}^{7} x_{2}^{7} x_{3} x_{4}^{2} x_{5}^{12}$ 252. $x_{1}^{7} x_{2}^{7} x_{3}^{8} x_{4} x_{5}^{6}$ 256. $x_{1}^{7} x_{2}^{9} x_{3}^{3} x_{4}^{6} x_{5}^{4}$ 260. $x_{1}^{7} x_{2}^{11} x_{3}^{5} x_{4}^{2} x_{5}^{4}$.

For $t \geqslant 4$,

1. $x_{1} x_{2} x_{3}^{2^{t}-2} x_{4}^{2^{t}-2} x_{5}^{2^{t+1}-1}$

4. $x_{1} x_{2}^{2^{2}-2} x_{3} x_{4}^{2^{t}-2} x_{5}^{2^{t+1}-1}$

7. $x_{1} x_{2}^{2^{t+1}-1} x_{3} x_{4}^{2^{t}-2} x_{5}^{2^{t}-2}$

10. $x_{1}^{2^{t+1}-1} x_{2} x_{3}^{2^{t}-2} x_{4} x_{5}^{2^{t}-2}$

13. $x_{1}^{3} x_{2}^{5} x_{3}^{2^{t+1}-1} x_{4}^{2^{t}-6} x_{5}^{2^{t}-4}$

16. $x_{1} x_{2}^{2} x_{3}^{2^{t}-3} x_{4}^{2^{t}-2} x_{5}^{2^{t+1}-1}$

19. $x_{1} x_{2}^{2^{t+1}-1} x_{3}^{2} x_{4}^{2^{t}-3} x_{5}^{2^{t}-2}$

22. $x_{1} x_{2}^{3} x_{3}^{2^{t}-4} x_{4}^{2^{t+1}-1} x_{5}^{2^{t}-2}$

25. $x_{1} x_{2}^{3} x_{3}^{2^{t+1}-1} x_{4}^{2^{t}-4} x_{5}^{2^{t}-2}$

28. $x_{1} x_{2}^{2^{t+1}-1} x_{3}^{3} x_{4}^{2^{t}-2} x_{5}^{2^{t}-4}$

31. $x_{1}^{3} x_{2} x_{3}^{2^{t}-2} x_{4}^{2^{t}-4} x_{5}^{2^{t+1}-1}$

34. $x_{1}^{3} x_{2} x_{3}^{2^{t+1}-1} x_{4}^{2^{t}-2} x_{5}^{2^{t}-4}$

37. $x_{1}^{2^{t+1}-1} x_{2} x_{3}^{3} x_{4}^{2^{t}-4} x_{5}^{2^{t}-2}$

40. $x_{1}^{2^{t+1}-1} x_{2}^{3} x_{3} x_{4}^{2^{t}-2} x_{5}^{2^{t}-4}$

43. $x_{1}^{3} x_{2}^{2^{t}-3} x_{3}^{2^{t+1}-1} x_{4}^{2} x_{5}^{2^{t}-4}$

46. $x_{1} x_{2}^{2} x_{3}^{2^{t}-4} x_{4}^{2^{t}-1} x_{5}^{2^{t+1}-1}$

49. $x_{1} x_{2}^{2} x_{3}^{2^{t}-1} x_{4}^{2^{t+1}-1} x_{5}^{2^{t}-4}$

52. $x_{1} x_{2}^{2^{t}-1} x_{3}^{2} x_{4}^{2^{t}-4} x_{5}^{2^{t+1}-1}$

55. $x_{1} x_{2}^{2^{t+1}-1} x_{3}^{2} x_{4}^{2^{t}-4} x_{5}^{2^{t}-1}$

58. $x_{1}^{2^{t}-1} x_{2} x_{3}^{2} x_{4}^{2^{t}-4} x_{5}^{2^{t+1}-1}$

61. $x_{1}^{2^{t}-1} x_{2}^{2^{t+1}-1} x_{3} x_{4}^{2} x_{5}^{2^{t}-4}$

64. $x_{1}^{2^{t+1}-1} x_{2} x_{3}^{2^{t}-1} x_{4}^{2} x_{5}^{2^{t}-4}$

67. $x_{1} x_{2} x_{3}^{2^{t}-2} x_{4}^{2^{t+1}-2} x_{5}^{2^{t}-1}$
2. $x_{1} x_{2} x_{3}^{2^{t}-2} x_{4}^{2^{t+1}-1} x_{5}^{2^{t}-2}$

5. $x_{1} x_{2}^{2^{t}-2} x_{3} x_{4}^{2^{t+1}-1} x_{5}^{2^{t}-2}$

8. $x_{1} x_{2}^{2^{t+1}-1} x_{3}^{2^{t}-2} x_{4} x_{5}^{2^{t}-2}$

11. $x_{1}^{3} x_{2}^{5} x_{3}^{2^{t}-6} x_{4}^{2^{t}-4} x_{5}^{2^{t+1}-1}$

14. $x_{1}^{3} x_{2}^{2^{t+1}-1} x_{3}^{5} x_{4}^{2^{t}-6} x_{5}^{2^{t}-4}$

17. $x_{1} x_{2}^{2} x_{3}^{2^{t}-3} x_{4}^{2^{t+1}-1} x_{5}^{2^{t}-2}$

20. $x_{1}^{2^{t+1}-1} x_{2} x_{3}^{2} x_{4}^{2^{t}-3} x_{5}^{2^{t}-2}$

23. $x_{1} x_{2}^{3} x_{3}^{2^{t}-2} x_{4}^{2^{t}-4} x_{5}^{2^{t+1}-1}$

26. $x_{1} x_{2}^{3} x_{3}^{2^{t+1}-1} x_{4}^{2^{t}-2} x_{5}^{2^{t}-4}$

29. $x_{1}^{3} x_{2} x_{3}^{2^{t}-4} x_{4}^{2^{t}-2} x_{5}^{2^{t+1}-1}$

32. $x_{1}^{3} x_{2} x_{3}^{2^{t}-2} x_{4}^{2^{t+1}-1} x_{5}^{2^{t}-4}$

35. $x_{1}^{3} x_{2}^{2^{t+1}-1} x_{3} x_{4}^{2^{t}-4} x_{5}^{2^{t}-2}$

38. $x_{1}^{2^{t+1}-1} x_{2} x_{3}^{3} x_{4}^{2^{t}-2} x_{5}^{2^{t}-4}$

41. $x_{1}^{3} x_{2}^{2^{t}-3} x_{3}^{2} x_{4}^{2^{t}-4} x_{5}^{2^{t+1}-1}$

44. $x_{1}^{3} x_{2}^{2^{t+1}-1} x_{3}^{2^{t}-3} x_{4}^{2} x_{5}^{2^{t}-4}$

47. $x_{1} x_{2}^{2} x_{3}^{2^{t}-4} x_{4}^{2^{t+1}-1} x_{5}^{2^{t}-1}$

50. $x_{1} x_{2}^{2} x_{3}^{2^{t+1}-1} x_{4}^{2^{t}-4} x_{5}^{2^{t}-1}$

53. $x_{1} x_{2}^{2^{t}-1} x_{3}^{2} x_{4}^{2^{t+1}-1} x_{5}^{2^{t}-4}$

56. $x_{1} x_{2}^{2^{t+1}-1} x_{3}^{2} x_{4}^{2^{t}-1} x_{5}^{2^{t}-4}$

59. $x_{1}^{2^{t}-1} x_{2} x_{3}^{2} x_{4}^{2^{t+1}-1} x_{5}^{2^{t}-4}$

62. $x_{1}^{2^{t+1}-1} x_{2} x_{3}^{2} x_{4}^{2^{t}-4} x_{5}^{2^{t}-1}$

65. $x_{1}^{2^{t+1}-1} x_{2}^{2^{t}-1} x_{3} x_{4}^{2} x_{5}^{2^{t}-4}$

68. $x_{1} x_{2} x_{3}^{2^{t}-1} x_{4}^{2^{t}-2} x_{5}^{2^{t+1}-2}$
3. $x_{1} x_{2} x_{3}^{2^{t+1}-1} x_{4}^{2^{t}-2} x_{5}^{2^{t}-2}$

6. $x_{1} x_{2}^{2^{t}-2} x_{3}^{2^{t+1}-1} x_{4} x_{5}^{2^{t}-2}$

9. $x_{1}^{2^{t+1}-1} x_{2} x_{3} x_{4}^{2^{t}-2} x_{5}^{2^{t}-2}$

12. $x_{1}^{3} x_{2}^{5} x_{3}^{2^{t}-6} x_{4}^{2^{t+1}-1} x_{5}^{2^{t}-4}$

15. $x_{1}^{2^{t+1}-1} x_{2}^{3} x_{3}^{5} x_{4}^{2^{t}-6} x_{5}^{2^{t}-4}$

18. $x_{1} x_{2}^{2} x_{3}^{2^{t+1}-1} x_{4}^{2^{t}-3} x_{5}^{2^{t}-2}$

21. $x_{1} x_{2}^{3} x_{3}^{2^{t}-4} x_{4}^{2^{t}-2} x_{5}^{2^{t+1}-1}$

24. $x_{1} x_{2}^{3} x_{3}^{2^{t}-2} x_{4}^{2^{t+1}-1} x_{5}^{2^{t}-4}$

27. $x_{1} x_{2}^{2^{t+1}-1} x_{3}^{3} x_{4}^{2^{t}-4} x_{5}^{2^{t}-2}$

30. $x_{1}^{3} x_{2} x_{3}^{2^{t}-4} x_{4}^{2^{t+1}-1} x_{5}^{2^{t}-2}$

33. $x_{1}^{3} x_{2} x_{3}^{2^{t+1}-1} x_{4}^{2^{t}-4} x_{5}^{2^{t}-2}$

36. $x_{1}^{3} x_{2}^{2^{t+1}-1} x_{3} x_{4}^{2^{t}-2} x_{5}^{2^{t}-4}$

39. $x_{1}^{2^{t+1}-1} x_{2}^{3} x_{3} x_{4}^{2^{t}-4} x_{5}^{2^{t}-2}$

42. $x_{1}^{3} x_{2}^{2^{t}-3} x_{3}^{2} x_{4}^{2^{t+1}-1} x_{5}^{2^{t}-4}$

45. $x_{1}^{2^{t+1}-1} x_{2}^{3} x_{3}^{2^{t}-3} x_{4}^{2} x_{5}^{2^{t}-4}$

48. $x_{1} x_{2}^{2} x_{3}^{2^{t}-1} x_{4}^{2^{t}-4} x_{5}^{2^{t+1}-1}$

51. $x_{1} x_{2}^{2} x_{3}^{2^{t+1}-1} x_{4}^{2^{t}-1} x_{5}^{2^{t}-4}$

54. $x_{1} x_{2}^{2^{t}-1} x_{3}^{2^{t+1}-1} x_{4}^{2} x_{5}^{2^{t}-4}$

57. $x_{1} x_{2}^{2^{t+1}-1} x_{3}^{2^{t}-1} x_{4}^{2} x_{5}^{2^{t}-4}$

60. $x_{1}^{2^{t}-1} x_{2} x_{3}^{2^{t+1}-1} x_{4}^{2} x_{5}^{2^{t}-4}$

63. $x_{1}^{2^{t+1}-1} x_{2} x_{3}^{2} x_{4}^{2^{t}-1} x_{5}^{2^{t}-4}$

66. $x_{1} x_{2} x_{3}^{2^{t}-2} x_{4}^{2^{t}-1} x_{5}^{2^{t+1}-2}$

69. $x_{1} x_{2} x_{3}^{2^{t}-1} x_{4}^{2^{t+1}-2} x_{5}^{2^{t}-2}$ 
70. $x_{1} x_{2} x_{3}^{2^{t+1}-2} x_{4}^{2^{t}-2} x_{5}^{2^{t}-1}$

73. $x_{1} x_{2}^{2^{t}-2} x_{3} x_{4}^{2^{t+1}-2} x_{5}^{2^{t}-1}$

76. $x_{1} x_{2}^{2^{t}-1} x_{3} x_{4}^{2^{t+1}-2} x_{5}^{2^{t}-2}$

79. $x_{1} x_{2}^{2^{t+1}-2} x_{3} x_{4}^{2^{t}-2} x_{5}^{2^{t}-1}$

82. $x_{1}^{2^{t}-1} x_{2} x_{3} x_{4}^{2^{t}-2} x_{5}^{2^{t+1}-2}$

85. $x_{1}^{2^{t}-1} x_{2} x_{3}^{2^{t+1}-2} x_{4} x_{5}^{2^{t}-2}$

88. $x_{1} x_{2}^{2} x_{3}^{2^{t}-1} x_{4}^{2^{t}-3} x_{5}^{2^{t+1}-2}$

91. $x_{1} x_{2}^{2} x_{3}^{2^{t}-1} x_{4}^{2^{t+1}-3} x_{5}^{2^{t}-2}$

94. $x_{1} x_{2}^{2^{t}-1} x_{3}^{2} x_{4}^{2^{t+1}-3} x_{5}^{2^{t}-2}$

97. $x_{1} x_{2}^{2} x_{3}^{2^{t}-1} x_{4}^{2^{t+1}-4} x_{5}^{2^{t}-1}$

100. $x_{1} x_{2}^{2^{t}-1} x_{3}^{2} x_{4}^{2^{t+1}-4} x_{5}^{2^{t}-1}$

103. $x_{1}^{2^{t}-1} x_{2} x_{3}^{2} x_{4}^{2^{t+1}-4} x_{5}^{2^{t}-1}$

106. $x_{1} x_{2}^{3} x_{3}^{2^{t}-4} x_{4}^{2^{t}-1} x_{5}^{2^{t+1}-2}$

109. $x_{1} x_{2}^{3} x_{3}^{2^{t}-1} x_{4}^{2^{t+1}-2} x_{5}^{2^{t}-4}$

112. $x_{1} x_{2}^{2^{t}-1} x_{3}^{3} x_{4}^{2^{t}-4} x_{5}^{2^{t+1}-2}$

115. $x_{1}^{3} x_{2} x_{3}^{2^{t}-4} x_{4}^{2^{t+1}-2} x_{5}^{2^{t}-1}$

118. $x_{1}^{3} x_{2} x_{3}^{2^{t+1}-2} x_{4}^{2^{t}-4} x_{5}^{2^{t}-1}$

121. $x_{1}^{3} x_{2}^{2^{t}-1} x_{3} x_{4}^{2^{t+1}-2} x_{5}^{2^{t}-4}$

124. $x_{1}^{2^{t}-1} x_{2}^{3} x_{3} x_{4}^{2^{t}-4} x_{5}^{2^{t+1}-2}$

127. $x_{1} x_{2}^{3} x_{3}^{2^{t}-3} x_{4}^{2^{t+1}-2} x_{5}^{2^{t}-2}$

130. $x_{1} x_{2}^{2^{t}-2} x_{3}^{3} x_{4}^{2^{t}-3} x_{5}^{2^{t+1}-2}$

133. $x_{1}^{3} x_{2} x_{3}^{2^{t}-3} x_{4}^{2^{t+1}-2} x_{5}^{2^{t}-2}$

136. $x_{1}^{3} x_{2}^{2^{t}-3} x_{3} x_{4}^{2^{t}-2} x_{5}^{2^{t+1}-2}$

139. $x_{1}^{3} x_{2}^{2^{t}-3} x_{3}^{2^{t+1}-2} x_{4} x_{5}^{2^{t}-2}$

142. $x_{1} x_{2}^{2^{t}-2} x_{3}^{3} x_{4}^{2^{t+1}-3} x_{5}^{2^{t}-2}$

145. $x_{1}^{3} x_{2}^{2^{t+1}-3} x_{3} x_{4}^{2^{t}-2} x_{5}^{2^{t}-2}$

148. $x_{1} x_{2}^{3} x_{3}^{2^{t}-2} x_{4}^{2^{t+1}-4} x_{5}^{2^{t}-1}$

151. $x_{1} x_{2}^{3} x_{3}^{2^{t+1}-4} x_{4}^{2^{t}-2} x_{5}^{2^{t}-1}$

154. $x_{1} x_{2}^{2^{t}-1} x_{3}^{3} x_{4}^{2^{t+1}-4} x_{5}^{2^{t}-2}$

157. $x_{1}^{3} x_{2} x_{3}^{2^{t}-1} x_{4}^{2^{t}-2} x_{5}^{2^{t+1}-4}$

160. $x_{1}^{3} x_{2} x_{3}^{2^{t+1}-4} x_{4}^{2^{t}-1} x_{5}^{2^{t}-2}$

163. $x_{1}^{2^{t}-1} x_{2} x_{3}^{3} x_{4}^{2^{t}-2} x_{5}^{2^{t+1}-4}$

166. $x_{1}^{2^{t}-1} x_{2}^{3} x_{3} x_{4}^{2^{t+1}-4} x_{5}^{2^{t}-2}$

169. $x_{1} x_{2}^{6} x_{3}^{2^{t+1}-5} x_{4}^{2^{t}-3} x_{5}^{2^{t}-2}$

172. $x_{1} x_{2}^{7} x_{3}^{2^{t}-6} x_{4}^{2^{t+1}-3} x_{5}^{2^{t}-2}$

175. $x_{1} x_{2}^{7} x_{3}^{2^{t}-5} x_{4}^{2^{t+1}-2} x_{5}^{2^{t}-4}$

178. $x_{1}^{7} x_{2}^{2^{t}-5} x_{3} x_{4}^{2^{t}-4} x_{5}^{2^{t+1}-2}$

181. $x_{1} x_{2}^{7} x_{3}^{2^{t}-5} x_{4}^{2^{t+1}-4} x_{5}^{2^{t}-2}$

184. $x_{1}^{7} x_{2}^{2^{t}-5} x_{3} x_{4}^{2^{t}-2} x_{5}^{2^{t+1}-4}$

187. $x_{1} x_{2}^{7} x_{3}^{2^{t+1}-5} x_{4}^{2^{t}-2} x_{5}^{2^{t}-4}$

190. $x_{1}^{7} x_{2}^{2^{t+1}-5} x_{3} x_{4}^{2^{t}-4} x_{5}^{2^{t}-2}$

193. $x_{1}^{7} x_{2} x_{3}^{2^{t+1}-6} x_{4}^{2^{t}-3} x_{5}^{2^{t}-2}$
71. $x_{1} x_{2} x_{3}^{2^{t+1}-2} x_{4}^{2^{t}-1} x_{5}^{2^{t}-2}$

74. $x_{1} x_{2}^{2^{t}-2} x_{3}^{2^{t}-1} x_{4} x_{5}^{2^{t+1}-2}$

77. $x_{1} x_{2}^{2^{t}-1} x_{3}^{2^{t}-2} x_{4} x_{5}^{2^{t+1}-2}$

80. $x_{1} x_{2}^{2^{t+1}-2} x_{3} x_{4}^{2^{t}-1} x_{5}^{2^{t}-2}$

83. $x_{1}^{2^{t}-1} x_{2} x_{3} x_{4}^{2^{t+1}-2} x_{5}^{2^{t}-2}$

86. $x_{1} x_{2}^{2} x_{3}^{2^{t}-3} x_{4}^{2^{t}-1} x_{5}^{2^{t+1}-2}$

89. $x_{1} x_{2}^{2^{t}-1} x_{3}^{2} x_{4}^{2^{t}-3} x_{5}^{2^{t+1}-2}$

92. $x_{1} x_{2}^{2} x_{3}^{2^{t+1}-3} x_{4}^{2^{t}-2} x_{5}^{2^{t}-1}$

95. $x_{1}^{2^{t}-1} x_{2} x_{3}^{2} x_{4}^{2^{t+1}-3} x_{5}^{2^{t}-2}$

98. $x_{1} x_{2}^{2} x_{3}^{2^{t+1}-4} x_{4}^{2^{t}-1} x_{5}^{2^{t}-1}$

101. $x_{1} x_{2}^{2^{t}-1} x_{3}^{2^{t}-1} x_{4}^{2} x_{5}^{2^{t+1}-4}$

104. $x_{1}^{2^{t}-1} x_{2} x_{3}^{2^{t}-1} x_{4}^{2} x_{5}^{2^{t+1}-4}$

107. $x_{1} x_{2}^{3} x_{3}^{2^{t}-4} x_{4}^{2^{t+1}-2} x_{5}^{2^{t}-1}$

110. $x_{1} x_{2}^{3} x_{3}^{2^{t+1}-2} x_{4}^{2^{t}-4} x_{5}^{2^{t}-1}$

113. $x_{1} x_{2}^{2^{t}-1} x_{3}^{3} x_{4}^{2^{t+1}-2} x_{5}^{2^{t}-4}$

116. $x_{1}^{3} x_{2} x_{3}^{2^{t}-1} x_{4}^{2^{t}-4} x_{5}^{2^{t+1}-2}$

119. $x_{1}^{3} x_{2} x_{3}^{2^{t+1}-2} x_{4}^{2^{t}-1} x_{5}^{2^{t}-4}$

122. $x_{1}^{2^{t}-1} x_{2} x_{3}^{3} x_{4}^{2^{t}-4} x_{5}^{2^{t+1}-2}$

125. $x_{1}^{2^{t}-1} x_{2}^{3} x_{3} x_{4}^{2^{t+1}-2} x_{5}^{2^{t}-4}$

128. $x_{1} x_{2}^{3} x_{3}^{2^{t}-2} x_{4}^{2^{t}-3} x_{5}^{2^{t+1}-2}$

131. $x_{1} x_{2}^{2^{t+1}-2} x_{3}^{3} x_{4}^{2^{t}-3} x_{5}^{2^{t}-2}$

134. $x_{1}^{3} x_{2} x_{3}^{2^{t}-2} x_{4}^{2^{t}-3} x_{5}^{2^{t+1}-2}$

137. $x_{1}^{3} x_{2}^{2^{t}-3} x_{3} x_{4}^{2^{t+1}-2} x_{5}^{2^{t}-2}$

140. $x_{1} x_{2}^{3} x_{3}^{2^{t}-2} x_{4}^{2^{t+1}-3} x_{5}^{2^{t}-2}$

143. $x_{1}^{3} x_{2} x_{3}^{2^{t}-2} x_{4}^{2^{t+1}-3} x_{5}^{2^{t}-2}$

146. $x_{1}^{3} x_{2}^{2^{t+1}-3} x_{3}^{2^{t}-2} x_{4} x_{5}^{2^{t}-2}$

149. $x_{1} x_{2}^{3} x_{3}^{2^{t}-1} x_{4}^{2^{t}-2} x_{5}^{2^{t+1}-4}$

152. $x_{1} x_{2}^{3} x_{3}^{2^{t+1}-4} x_{4}^{2^{t}-1} x_{5}^{2^{t}-2}$

155. $x_{1}^{3} x_{2} x_{3}^{2^{t}-2} x_{4}^{2^{t}-1} x_{5}^{2^{t+1}-4}$

158. $x_{1}^{3} x_{2} x_{3}^{2^{t}-1} x_{4}^{2^{t+1}-4} x_{5}^{2^{t}-2}$

161. $x_{1}^{3} x_{2}^{2^{t}-1} x_{3} x_{4}^{2^{t}-2} x_{5}^{2^{t+1}-4}$

164. $x_{1}^{2^{t}-1} x_{2} x_{3}^{3} x_{4}^{2^{t+1}-4} x_{5}^{2^{t}-2}$

167. $x_{1} x_{2}^{6} x_{3}^{2^{t}-5} x_{4}^{2^{t}-3} x_{5}^{2^{t+1}-2}$

170. $x_{1} x_{2}^{7} x_{3}^{2^{t}-6} x_{4}^{2^{t}-3} x_{5}^{2^{t+1}-2}$

173. $x_{1}^{7} x_{2} x_{3}^{2^{t}-6} x_{4}^{2^{t+1}-3} x_{5}^{2^{t}-2}$

176. $x_{1}^{7} x_{2} x_{3}^{2^{t}-5} x_{4}^{2^{t}-4} x_{5}^{2^{t+1}-2}$

179. $x_{1}^{7} x_{2}^{2^{t}-5} x_{3} x_{4}^{2^{t+1}-2} x_{5}^{2^{t}-4}$

182. $x_{1}^{7} x_{2} x_{3}^{2^{t}-5} x_{4}^{2^{t}-2} x_{5}^{2^{t+1}-4}$

185. $x_{1}^{7} x_{2}^{2^{t}-5} x_{3} x_{4}^{2^{t+1}-4} x_{5}^{2^{t}-2}$

188. $x_{1}^{7} x_{2} x_{3}^{2^{t+1}-5} x_{4}^{2^{t}-4} x_{5}^{2^{t}-2}$

191. $x_{1}^{7} x_{2}^{2^{t+1}-5} x_{3} x_{4}^{2^{t}-2} x_{5}^{2^{t}-4}$

194. $x_{1}^{3} x_{2}^{2^{t}-1} x_{3}^{2^{t+1}-3} x_{4}^{2} x_{5}^{2^{t}-4}$
72. $x_{1} x_{2}^{2^{t}-2} x_{3} x_{4}^{2^{t}-1} x_{5}^{2^{t+1}-2}$

75. $x_{1} x_{2}^{2^{t}-1} x_{3} x_{4}^{2^{t}-2} x_{5}^{2^{t+1}-2}$

78. $x_{1} x_{2}^{2^{t}-1} x_{3}^{2^{t+1}-2} x_{4} x_{5}^{2^{t}-2}$

81. $x_{1} x_{2}^{2^{t+1}-2} x_{3}^{2^{t}-1} x_{4} x_{5}^{2^{t}-2}$

84. $x_{1}^{2^{t}-1} x_{2} x_{3}^{2^{t}-2} x_{4} x_{5}^{2^{t+1}-2}$

87. $x_{1} x_{2}^{2} x_{3}^{2^{t}-3} x_{4}^{2^{t+1}-2} x_{5}^{2^{t}-1}$

90. $x_{1}^{2^{t}-1} x_{2} x_{3}^{2} x_{4}^{2^{t}-3} x_{5}^{2^{t+1}-2}$

93. $x_{1} x_{2}^{2} x_{3}^{2^{t+1}-3} x_{4}^{2^{t}-1} x_{5}^{2^{t}-2}$

96. $x_{1} x_{2}^{2} x_{3}^{2^{t}-1} x_{4}^{2^{t}-1} x_{5}^{2^{t+1}-4}$

99. $x_{1} x_{2}^{2^{t}-1} x_{3}^{2} x_{4}^{2^{t}-1} x_{5}^{2^{t+1}-4}$

102. $x_{1}^{2^{t}-1} x_{2} x_{3}^{2} x_{4}^{2^{t}-1} x_{5}^{2^{t+1}-4}$

105. $x_{1}^{2^{t}-1} x_{2}^{2^{t}-1} x_{3} x_{4}^{2} x_{5}^{2^{t+1}-4}$

108. $x_{1} x_{2}^{3} x_{3}^{2^{t}-1} x_{4}^{2^{t}-4} x_{5}^{2^{t+1}-2}$

111. $x_{1} x_{2}^{3} x_{3}^{2^{t+1}-2} x_{4}^{2^{t}-1} x_{5}^{2^{t}-4}$

114. $x_{1}^{3} x_{2} x_{3}^{2^{t}-4} x_{4}^{2^{t}-1} x_{5}^{2^{t+1}-2}$

117. $x_{1}^{3} x_{2} x_{3}^{2^{t}-1} x_{4}^{2^{t+1}-2} x_{5}^{2^{t}-4}$

120. $x_{1}^{3} x_{2}^{2^{t}-1} x_{3} x_{4}^{2^{t}-4} x_{5}^{2^{t+1}-2}$

123. $x_{1}^{2^{t}-1} x_{2} x_{3}^{3} x_{4}^{2^{t+1}-2} x_{5}^{2^{t}-4}$

126. $x_{1} x_{2}^{3} x_{3}^{2^{t}-3} x_{4}^{2^{t}-2} x_{5}^{2^{t+1}-2}$

129. $x_{1} x_{2}^{3} x_{3}^{2^{t+1}-2} x_{4}^{2^{t}-3} x_{5}^{2^{t}-2}$

132. $x_{1}^{3} x_{2} x_{3}^{2^{t}-3} x_{4}^{2^{t}-2} x_{5}^{2^{t+1}-2}$

135. $x_{1}^{3} x_{2} x_{3}^{2^{t+1}-2} x_{4}^{2^{t}-3} x_{5}^{2^{t}-2}$

138. $x_{1}^{3} x_{2}^{2^{t}-3} x_{3}^{2^{t}-2} x_{4} x_{5}^{2^{t+1}-2}$

141. $x_{1} x_{2}^{3} x_{3}^{2^{t+1}-3} x_{4}^{2^{t}-2} x_{5}^{2^{t}-2}$

144. $x_{1}^{3} x_{2} x_{3}^{2^{t+1}-3} x_{4}^{2^{t}-2} x_{5}^{2^{t}-2}$

147. $x_{1} x_{2}^{3} x_{3}^{2^{t}-2} x_{4}^{2^{t}-1} x_{5}^{2^{t+1}-4}$

150. $x_{1} x_{2}^{3} x_{3}^{2^{t}-1} x_{4}^{2^{t+1}-4} x_{5}^{2^{t}-2}$

153. $x_{1} x_{2}^{2^{t}-1} x_{3}^{3} x_{4}^{2^{t}-2} x_{5}^{2^{t+1}-4}$

156. $x_{1}^{3} x_{2} x_{3}^{2^{t}-2} x_{4}^{2^{t+1}-4} x_{5}^{2^{t}-1}$

159. $x_{1}^{3} x_{2} x_{3}^{2^{t+1}-4} x_{4}^{2^{t}-2} x_{5}^{2^{t}-1}$

162. $x_{1}^{3} x_{2}^{2^{t}-1} x_{3} x_{4}^{2^{t+1}-4} x_{5}^{2^{t}-2}$

165. $x_{1}^{2^{t}-1} x_{2}^{3} x_{3} x_{4}^{2^{t}-2} x_{5}^{2^{t+1}-4}$

168. $x_{1} x_{2}^{6} x_{3}^{2^{t}-5} x_{4}^{2^{t+1}-3} x_{5}^{2^{t}-2}$

171. $x_{1}^{7} x_{2} x_{3}^{2^{t}-6} x_{4}^{2^{t}-3} x_{5}^{2^{t+1}-2}$

174. $x_{1} x_{2}^{7} x_{3}^{2^{t}-5} x_{4}^{2^{t}-4} x_{5}^{2^{t+1}-2}$

177. $x_{1}^{7} x_{2} x_{3}^{2^{t}-5} x_{4}^{2^{t+1}-2} x_{5}^{2^{t}-4}$

180. $x_{1} x_{2}^{7} x_{3}^{2^{t}-5} x_{4}^{2^{t}-2} x_{5}^{2^{t+1}-4}$

183. $x_{1}^{7} x_{2} x_{3}^{2^{t}-5} x_{4}^{2^{t+1}-4} x_{5}^{2^{t}-2}$

186. $x_{1} x_{2}^{7} x_{3}^{2^{t+1}-5} x_{4}^{2^{t}-4} x_{5}^{2^{t}-2}$

189. $x_{1}^{7} x_{2} x_{3}^{2^{t+1}-5} x_{4}^{2^{t}-2} x_{5}^{2^{t}-4}$

192. $x_{1} x_{2}^{7} x_{3}^{2^{t+1}-6} x_{4}^{2^{t}-3} x_{5}^{2^{t}-2}$

195. $x_{1}^{3} x_{2}^{2^{t+1}-3} x_{3}^{2} x_{4}^{2^{t}-4} x_{5}^{2^{t}-1}$ 


196. $x_{1}^{3} x_{2}^{2^{t+1}-3} x_{3}^{2} x_{4}^{2^{t}-1} x_{5}^{2^{t}-4}$
199. $x_{1}^{3} x_{2}^{2^{t}-3} x_{3}^{2} x_{4}^{2^{t}-3} x_{5}^{2^{t+1}-2}$
202. $x_{1}^{3} x_{2}^{2^{t}-3} x_{3}^{2} x_{4}^{2^{t}-1} x_{5}^{2^{t+1}-4}$
205. $x_{1}^{3} x_{2}^{2^{t}-1} x_{3}^{2^{t}-3} x_{4}^{2} x_{5}^{2^{t+1}-4}$
208. $x_{1}^{7} x_{2}^{2^{t}-5} x_{3}^{2^{2+1}-3} x_{4}^{2} x_{5}^{2^{t}-4}$
211. $x_{1}^{3} x_{2}^{3} x^{2^{t}-3} x_{4}^{2^{t}-4} x_{5}^{2^{t+1}-2}$
214. $x_{1}^{3} x_{2}^{2^{t}-3} x_{3}^{3} x_{4}^{2^{t+1}-2} x_{5}^{2^{t}-4}$
217. $x_{1}^{3} x_{2}^{3} x_{3}^{2^{t+1}-3} x_{4}^{2^{t}-2} x_{5}^{2^{t}-4}$
220. $x_{1}^{3} x_{2}^{3} x_{3}^{2^{t}-3} x_{4}^{2^{-}-2} x_{5}^{2^{t+1}-4}$
223. $x_{1}^{3} x_{2}^{2^{t}-3} x_{3}^{3} x_{4}^{2^{t}-2} x_{5}^{2^{t+1}-4}$
226. $x_{1}^{3} x_{2}^{4} x_{3}^{2^{t}-5} x_{4}^{2^{t+1}-3} x_{5}^{2^{t}-2}$
229. $x_{1}^{3} x_{2}^{5} x_{3}^{2^{t}-6} x_{4}^{2^{t+1}-3} x_{5}^{2^{t}-2}$
232. $x_{1}^{3} x_{2}^{5} x_{3}^{2^{t}-1} x_{4}^{2^{t}-6} x_{5}^{2^{t+1}-4}$
235. $x_{1}^{3} x_{2}^{5} x_{3}^{2^{t}-5} x_{4}^{2^{t}-4} x_{5}^{2^{t+1}-2}$
238. $x_{1}^{3} x_{2}^{5} x_{3}^{2^{t}-5} x_{4}^{2^{t+1}-4} x_{5}^{2^{t}-2}$
241. $x_{1}^{3} x_{2}^{5} x_{3}^{2^{t}-1} x_{4}^{2^{t+1}-6} x_{5}^{2^{t}-4}$
244. $x_{1}^{3} x_{2}^{2^{t}-1} x_{3}^{5} x_{4}^{2^{t+1}-6} x_{5}^{2^{t}-4}$
247. $x_{1}^{3} x_{2}^{7} x_{3}^{2^{t}-7} x_{4}^{2^{t}-4} x_{5}^{2^{t+1}-2}$
250. $x_{1}^{7} x_{2}^{3} x_{3}^{2^{t}-7} x_{4}^{2^{t+1}-2} x_{5}^{2^{t}-4}$
253. $x_{1}^{7} x_{2}^{3} x_{3}^{2^{t}-7} x_{4}^{2^{t}-2} x_{5}^{2^{t+1}-4}$
256. $x_{1}^{7} x_{2}^{3} x_{3}^{2^{t+1}-3} x_{4}^{2^{t}-6} x_{5}^{2^{t}-4}$
259. $x_{1}^{3} x_{2}^{7} x_{3}^{2^{t}-3} x_{4}^{2^{+1}-6} x_{5}^{2^{t}-4}$
262. $x_{1}^{7} x_{2}^{3} x_{3}^{2^{t+1}-7} x_{4}^{2^{t}-4} x_{5}^{2^{t}-2}$
265. $x_{1}^{7} x_{2}^{2^{t}-5} x_{3}^{5} x_{4}^{2^{-6}} x_{5}^{2^{t+1}-4}$
268. $x_{1}^{7} x_{2}^{2^{t+1}-5} x_{3}^{5} x_{4}^{2^{t}-6} x_{5}^{2^{t}-4}$

197. $x_{1}^{3} x_{2}^{2^{t+1}-3} x_{3}^{2^{t}-1} x_{4}^{2} x_{5}^{2^{t}-4}$

200. $x_{1}^{3} x_{2}^{2^{t}-3} x_{3}^{2} x_{4}^{2^{t+1}-3} x_{5}^{2^{t}-2}$

203. $x_{1}^{3} x_{2}^{2^{t}-3} x_{3}^{2} x_{4}^{2^{t+1}-4} x_{5}^{2^{t}-1}$

206. $x_{1}^{2^{t}-1} x_{2}^{3} x_{3}^{2^{t}-3} x_{4}^{2} x_{5}^{2^{t+1}-4}$

209. $x_{1}^{7} x_{2}^{2^{t+1}-5} x_{3}^{2^{t}-3} x_{4}^{2} x_{5}^{2^{t}-4}$

212. $x_{1}^{3} x_{2}^{3} x_{3}^{2^{t}-3} x_{4}^{2^{t+1}-2} x_{5}^{2^{t}-4}$

215. $x_{1}^{3} x_{2}^{3} x_{3}^{2^{t}-4} x_{4}^{2^{t+1}-3} x_{5}^{2^{t}-2}$

218. $x_{1}^{3} x_{2}^{2^{t+1}-3} x_{3}^{3} x_{4}^{2^{t}-4} x_{5}^{2^{t}-2}$

221. $x_{1}^{3} x_{2}^{3} x_{3}^{2^{t}-3} x_{4}^{2^{t+1}-4} x_{5}^{2^{t}-2}$

224. $x_{1}^{3} x_{2}^{2^{t}-3} x_{3}^{3} x_{4}^{2^{t+1}-4} x_{5}^{2^{t}-2}$

227. $x_{1}^{3} x_{2}^{4} x_{3}^{2^{t+1}-5} x_{4}^{2^{t}-3} x_{5}^{2^{t}-2}$

230. $x_{1}^{3} x_{2}^{5} x_{3}^{2^{t}-6} x_{4}^{2^{t}-1} x_{5}^{2^{t+1}-4}$

233. $x_{1}^{3} x_{2}^{2^{t}-1} x_{3}^{5} x_{4}^{2^{t}-6} x_{5}^{2^{t+1}-4}$

236. $x_{1}^{3} x_{2}^{5} x_{3}^{2^{t}-5} x_{4}^{2^{t+1}-2} x_{5}^{2^{t}-4}$

239. $x_{1}^{3} x_{2}^{5} x_{3}^{2^{t+1}-5} x_{4}^{2^{t}-4} x_{5}^{2^{t}-2}$

242. $x_{1}^{3} x_{2}^{5} x_{3}^{2^{t+1}-6} x_{4}^{2^{t}-4} x_{5}^{2^{t}-1}$

245. $x_{1}^{2^{t}-1} x_{2}^{3} x_{3}^{5} x_{4}^{2^{t+1}-6} x_{5}^{2^{t}-4}$

248. $x_{1}^{3} x_{2}^{7} x_{3}^{2^{t}-7} x_{4}^{2^{t+1}-2} x_{5}^{2^{t}-4}$

251. $x_{1}^{3} x_{2}^{7} x_{3}^{2^{t}-7} x_{4}^{2^{t}-2} x_{5}^{2^{t+1}-4}$

254. $x_{1}^{7} x_{2}^{3} x_{3}^{2^{t}-7} x_{4}^{2^{t+1}-4} x_{5}^{2^{t}-2}$

257. $x_{1}^{3} x_{2}^{7} x_{3}^{2^{t}-3} x_{4}^{2^{t}-6} x_{5}^{2^{t+1}-4}$

260. $x_{1}^{7} x_{2}^{3} x_{3}^{2^{t}-3} x_{4}^{2^{t+1}-6} x_{5}^{2^{t}-4}$

263. $x_{1}^{3} x_{2}^{15} x_{3}^{2^{t+1}-11} x_{4}^{2^{t}-6} x_{5}^{2^{t}-4}$

266. $x_{1}^{7} x_{2}^{2^{t}-5} x_{3}^{5} x_{4}^{2^{t+1}-6} x_{5}^{2^{t}-4}$
198. $x_{1}^{2^{t}-1} x_{2}^{3} x_{3}^{2^{t+1}-3} x_{4}^{2} x_{5}^{2^{t}-4}$

201. $x_{1}^{3} x_{2}^{2^{t+1}-3} x_{3}^{2} x_{4}^{2^{t}-3} x_{5}^{2^{t}-2}$

204. $x_{1}^{3} x_{2}^{2^{t}-3} x_{3}^{2^{t}-1} x_{4}^{2} x_{5}^{2^{t+1}-4}$

207. $x_{1}^{7} x_{2}^{2^{t}-5} x_{3}^{2^{t}-3} x_{4}^{2} x_{5}^{2^{t+1}-4}$

210. $x_{1}^{3} x_{2}^{3} x_{3}^{2^{t}-4} x_{4}^{2^{t}-3} x_{5}^{2^{t+1}-2}$

213. $x_{1}^{3} x_{2}^{2^{t}-3} x_{3}^{3} x_{4}^{2^{t}-4} x_{5}^{2^{t+1}-2}$

216. $x_{1}^{3} x_{2}^{3} x_{3}^{2^{t+1}-3} x_{4}^{2^{t}-4} x_{5}^{2^{t}-2}$

219. $x_{1}^{3} x_{2}^{2^{t+1}-3} x_{3}^{3} x_{4}^{2^{t}-2} x_{5}^{2^{t}-4}$

222. $x_{1}^{3} x_{2}^{3} x_{3}^{2^{t+1}-4} x_{4}^{2^{t}-3} x_{5}^{2^{t}-2}$

225. $x_{1}^{3} x_{2}^{4} x_{3}^{2^{t}-5} x_{4}^{2^{t}-3} x_{5}^{2^{t+1}-2}$

228. $x_{1}^{3} x_{2}^{5} x_{3}^{2^{t}-6} x_{4}^{2^{t}-3} x_{5}^{2^{t+1}-2}$

231. $x_{1}^{3} x_{2}^{5} x_{3}^{2^{t}-6} x_{4}^{2^{t+1}-4} x_{5}^{2^{t}-1}$

234. $x_{1}^{2^{t}-1} x_{2}^{3} x_{3}^{5} x_{4}^{2^{t}-6} x_{5}^{2^{t+1}-4}$

237. $x_{1}^{3} x_{2}^{5} x_{3}^{2^{t}-5} x_{4}^{2^{t}-2} x_{5}^{2^{t+1}-4}$

240. $x_{1}^{3} x_{2}^{5} x_{3}^{2^{t+1}-5} x_{4}^{2^{t}-2} x_{5}^{2^{t}-4}$

243. $x_{1}^{3} x_{2}^{5} x_{3}^{2^{t+1}-6} x_{4}^{2^{t}-1} x_{5}^{2^{t}-4}$

246. $x_{1}^{3} x_{2}^{5} x_{3}^{2^{t+1}-6} x_{4}^{2^{t}-3} x_{5}^{2^{t}-2}$

249. $x_{1}^{7} x_{2}^{3} x_{3}^{2^{t}-7} x_{4}^{2^{t}-4} x_{5}^{2^{t+1}-2}$

252. $x_{1}^{3} x_{2}^{7} x_{3}^{2^{t}-7} x_{4}^{2^{t+1}-4} x_{5}^{2^{t}-2}$

255. $x_{1}^{3} x_{2}^{7} x_{3}^{2^{t+1}-3} x_{4}^{2^{t}-6} x_{5}^{2^{t}-4}$

258. $x_{1}^{7} x_{2}^{3} x_{3}^{2^{t}-3} x_{4}^{2^{t}-6} x_{5}^{2^{t+1}-4}$

261. $x_{1}^{3} x_{2}^{7} x_{3}^{2^{t+1}-7} x_{4}^{2^{t}-4} x_{5}^{2^{t}-2}$

264. $x_{1}^{15} x_{2}^{3} x_{3}^{2^{t+1}-11} x_{4}^{2^{t}-6} x_{5}^{2^{t}-4}$

267. $x_{1}^{7} x_{2}^{11} x_{3}^{2^{t+1}-11} x_{4}^{2^{t}-6} x_{5}^{2^{t}-4}$

For $t=4$,

$$
\text { 269. } x_{1}^{7} x_{2}^{11} x_{3}^{13} x_{4}^{14} x_{5}^{16} \quad \text { 270. } x_{1}^{7} x_{2}^{11} x_{3}^{13} x_{4}^{18} x_{5}^{12}
$$

For $t \geqslant 5$,

$$
\text { 269. } x_{1}^{7} x_{2}^{11} x_{3}^{2^{t}-11} x_{4}^{2^{t}-6} x_{5}^{2^{t+1}-4} \quad \text { 270. } x_{1}^{7} x_{2}^{11} x_{3}^{2^{t}-11} x_{4}^{2^{t+1}-6} x_{5}^{2^{t}-4} \text {. }
$$

\section{References}

[1] J. F. Adams, On the structure and applications of the Steenrod algebra, Comment. Math. Helv., 72 (1958), $180-214$.

[2] J. M. Boardman, Modular representations on the homology of power of real projective space, in: M. C. Tangora (Ed.), Algebraic Topology, Oaxtepec, 1991, in: Contemp. Math., 146 (1993), 49-70.

[3] W. Browder, The Kervaire invariant of framed manifolds and its generalization, Ann. of Math. (2) 90 (1969), 157-186.

[4] R. R. Bruner, L. M. Hà and N. H. V. Hưng, On behavior of the algebraic transfer, Trans. Amer. Math. Soc., 357 (2005), 437-487.

[5] M. C. Crabb and J. R. Hubbuck, Representations of the homology of BV and the Steenrod algebra II, Algebra Topology: new trend in localization and periodicity, Progr. Math., 136 (1996), 143-154.

[6] T. W. Chen, Determination of $\operatorname{Ext}_{\mathscr{A}}^{5, *}(\mathbb{Z} / 2, \mathbb{Z} / 2)$, Topol. Appl., 158 (2011), 660-689.

[7] P. H. Chơn and L. M. Hà, On May spectral sequences and the algebraic transfer, Manuscripta Math., 138 (2012), $141-160$.

[8] P. H. Chơn and L. M. Hà, On the May spectral sequence and the algebraic transfer II, Topol. Appl., 178 (2014), 372-383.

[9] L. M. Hà, Sub-Hopf algebras of the Steenrod algebra and the Singer transfer, in: Proceedings of the School and Conference in Algebraic Topology, Hà Nội 2004, in: Geom. Topol. Publ., 11 (2007), 101-124.

[10] M. A. Hill, M. J. Hopkins and D. C. Ravenel, On the non-existence of elements of kervaire invariant one, Ann. of Math. (2) 184 (2016), 1-262.

[11] N. H. V. Hưng, The cohomology of the Steenrod algebra and representations of the general linear groups, Trans. Amer. Math. Soc., 357 (2005), 4065-4089. 
[12] M. Kameko, Products of projective spaces as Steenrod modules, PhD. Thesis, The Johns Hopkins University, ProQuest LLC, Ann Arbor, MI, 1990.

[13] W.H. Lin and M. Mahowald, The Adams spectral sequence for Minami's Theorem, in: Proceedings of the Northwestern Homotopy Theory Conference (Providence, Rhode Island) (H. R. Miller and S. B. Priddy, eds.), Contemp. Math. vol. 220, 1983, pp. 143-177.

[14] W.H. Lin, $\operatorname{Ext}_{\mathscr{A}}^{4, *}(\mathbb{Z} / 2, \mathbb{Z} / 2)$ and $\operatorname{Ext}_{\mathscr{A}}^{5, *}(\mathbb{Z} / 2, \mathbb{Z} / 2)$, Topology Appl., 155 (2008), 459-496.

[15] M. Mahowald, A new infinite family in ${ }_{2} \pi_{*}^{S}$, Topology 16 (1977), 249-256.

[16] N. Minami, The Adams spectral sequence and the triple transfer, Amer. J. Math. 117 (1995), 965-985.

[17] N. Minami, The iterated transfer analogue of the new doomsday conjecture, Trans. Amer. Math. Soc. 351 (1999), $2325-2351$.

[18] S.A. Mitchell, Splitting $B(\mathbb{Z} / p)^{n}$ and BT ${ }^{n}$ Via Modular Representation Theory, Math. Z. 189 (1985), 1-9.

[19] M. F. Mothebe, Generators of the polynomial algebra $\mathbb{F}_{2}\left[x_{1}, x_{2}, \ldots, x_{n}\right]$ as a module over the Steenrod algebra, PhD. thesis, The University of Manchester, 1997.

[20] M. F. Mothebe, The admissible monomial basis for the polynomial algebra in degree thirteen, East-West J. of Mathematics, 18 (2016), 151-170.

[21] T. N. Nam, A-générateurs génériques pour l'algèbre polynomiale, Adv. Math., 186 (2004), 334-362.

[22] T.N. Nam, Transfert algébrique et action du groupe linéaire sur les puissances divisées modulo 2, Ann. Inst. Fourier (Grenoble) 58 (2008), 1785-1837.

[23] F. P. Peterson, Generators of $H^{*}\left(\mathbb{R} P^{\infty} \times \mathbb{R} P^{\infty}\right)$ as a module over the Steenrod algebra, Abstracts Amer. Math. Soc., No. 833, April 1987.

[24] Đ. V. Phúc and N. Sum, On the generators of the polynomial algebra as a module over the Steenrod algebra, C.R.Math. Acad. Sci. Paris, 353 (2015), 1035-1040.

[25] Đ. V. Phúc and N. Sum, On a minimal set of generators for the polynomial algebra of five variables as a module over the Steenrod algebra, Acta Math. Vietnam., 42 (2017), 149-162.

[26] Đ. V. Phúc, The hit problem for the polynomial algebra of five variables in degree seventeen and its application, East-West J. Math., 18 (2016), 27-46.

[27] Đ. V. Phúc, $\mathcal{A}$-generators for the polynomial algebra of five variables in degree $5\left(2^{t}-1\right)+6.2^{t}$, Commun. Korean Math. Soc., 35 (2020), 371-399.

[28] Đ.V. Phúc, On Peterson's open problem and representations of the general linear groups, J. Korean Math. Soc. 58 (2021), 643-702.

[29] Đ.V. Phúc, On modules over the mod 2 Steenrod algebra and hit problems, Preprint (2022), 40 pages, available online at https://www.researchgate.net/publication/365597765.

[30] Đ.V. Phúc, A note on the hit problem for the polynomial algebra of six variables and the sixth algebraic transfer, J. Algebra 613 (2023), https://doi.org/10.1016/j.jalgebra.2022.08.028.

[31] Đ.V. Phúc, On the hit problem for the algebra $H^{*}\left(B \mathbb{F}_{2}^{s}, \mathbb{F}_{2}\right)$ as a module over the mod two Steenrod algebra and its applications, Preprint (2022), available online at https://www.researchgate.net/publication/350236648.

[32] Đ.V. Phúc, On the dimension of $H^{*}\left(\left(\mathbb{Z}_{2}\right)^{\times t}, \mathbb{Z}_{2}\right)$ as a module over Steenrod ring, Preprint (2021) ,submitted for publication, available online at https://www.researchgate.net/publication/350063962.

[33] Đ.V. Phúc, Structure of the space of $G L_{4}\left(\mathbb{Z}_{2}\right)$-coinvariants $\mathbb{Z}_{2} \otimes_{G L_{4}\left(\mathbb{Z}_{2}\right)} P H_{*}\left(\mathbb{Z}_{2}^{4}, \mathbb{Z}_{2}\right)$ in some generic degrees and its application, Topol. Appl. (2022), accepted for publication, available online at https://www.researchgate.net/publication/ 350592289.

[34] Đ.V. Phúc, A note on the modular representation on the $\mathbb{Z} / 2$-homology groups of the fourth power of real projective space and its application, Preprint (2021), available online at https://www.researchgate.net/publication/350988977.

[35] Đ.V. Phúc, On the lambda algebra and Singer's cohomological transfer, Preprint (2021), available online at https://www. researchgate.net/publication/352017781.

[36] Đ.V. Phúc, The affirmative answer to Singer's conjecture on the algebraic transfer of rank four, Proc. Roy. Soc. Edinburgh Sect. A (2022), DOI: 10.1017/prm.2022.57, available online at https://www.researchgate.net/publication/352284459.

[37] S. Priddy, On characterizing summands in the classifying space of a group, I, Amer. Jour. Math., 112 (1990), 737-748.

[38] J. Repka and P. Selick, On the subalgebra of $H_{*}\left(\left(\mathbb{R} P^{\infty}\right)^{n} ; \mathbb{F}_{2}\right)$ annihilated by Steenrod operations, J. Pure Appl. Algebra, 127 (1998), 273-288.

[39] J. H. Silvermn, Hit polynomials and the canonical antiautomorphism of the Steenrod algebra, Proc. Amer. Math. Soc., 123 (1995), 627-637.

[40] J. H. Silverman and W. M. Singer, On the action of Steenrod squares on polynomial algebras II, J. Pure Appl. Algebra, 98 (1995), 95-103.

[41] W. M. Singer, The transfer in homological algebra, Math. Z., 202 (1989), 493-523.

[42] W. M. Singer, On the action of the Steenrod squares on polynomial algebras, Proc. Amer. Math. Soc., 111 (1991), $577-583$.

[43] N. E. Steenrod and D. B. A. Epstein, Cohomology operations,Annals of Mathematics Studies 50, Princeton University Press, Princeton N.J, 1962.

[44] N. Sum, The negative answer to Kameko's conjecture on the hit problem, Adv. Math., 225 (2010), 2365-2390.

[45] N. Sum, On the hit problem for the polynomial algebra, C. R. Math. Acad. Sci. Paris, 351 (2013) 565-568.

[46] N. Sum, On the Peterson hit problem of five variables and its applications to the fifth Singer transfer, East-West J. of Mathematics, 16 (2014), 47-62.

[47] N. Sum, On the Peterson hit problem, Adv. Math., 274 (2015), 432-489.

[48] N. Sum, The hit problem and the algebraic transfer in some degrees, East-West J. of Mathematics, 20 (2018), 158-179. 
[49] N. Sum, On the determination of the Singer transfer, Vietnam Journal of Science, Technology and Engineering, 60 (2018), 3-16.

[50] N. Sum, The squaring operation and the Singer algebraic transfer, Vietnam J. Math. (2020), DOI: 10.1007/s10013-020-00423-1.

[51] M. C. Tangora, On the cohomology of the Steenrod algebra, Math. Z., 116 (1970), 18-64.

[52] G. Walker and R. M. W. Wood, Young tableaux and the Steenrod algebra, in: Proceedings of the International School and Conference in Algebraic Topology, Hà Nội 2004, Geom. Topol. Monogr., Geom. Topol. Publ., Coventry, 11 (2007), $379-397$.

[53] G. Walker and R. M. W. Wood, Weyl modules and the mod 2 Steenrod algebra, J. Algebra, 311 (2007), 840-858.

[54] G. Walker and R. M. W. Wood, Polynomials and the mod 2 Steenrod Algebra: Volume 1, The Peterson hit problem; In: London Math. Soc. Lecture Note Ser., Cambridge Univ. Press, January 11, 2018.

[55] R. M. W. Wood, Steenrod squares of polynomials and the Peterson conjecture, Math. Proc. Cambriges Phil. Soc., 105 (1989), 307-309.

[56] R. M. W. Wood, Problems in the Steenrod algebra, Bull. London Math. Soc., 30 (1998), 449-517. 